(Prof. Dr. med. G. Hasenfuß)

im Zentrum Innere Medizin

der Medizinischen Fakultät der Universität Göttingen

\title{
Zusammenhang zwischen körperlicher Aktivität und gesteigerter sympathischer Nervenaktivität bei chronisch obstruktiver Lungenerkrankung
}

\author{
INAUGURAL-DISSERTATION \\ zur Erlangung des Doktorgrades \\ der Medizinischen Fakultät \\ der Georg-August-Universität zu Göttingen
}

\author{
vorgelegt von \\ Jan Folle \\ aus \\ Göttingen
}

Göttingen 2015 
Dekan:

1. Berichterstatter: Prof. Dr. med. T. Raupach

2. Berichterstatter: Prof. Dr. med. J. Hinz

Tag der mündlichen Prüfung: $\quad$ 16.06.2015 
Anmerkung: Aus Gründen der besseren Lesbarkeit wird in dieser Arbeit ausschließlich die männliche Sprachform verwendet. Dies impliziert aber auch die weibliche Form. 


\section{Inhaltsverzeichnis}

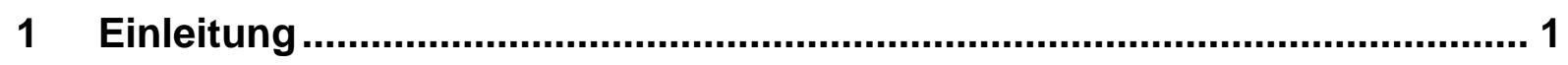

1.1 Die chronisch obstruktive Lungenerkrankung ....................................... 1

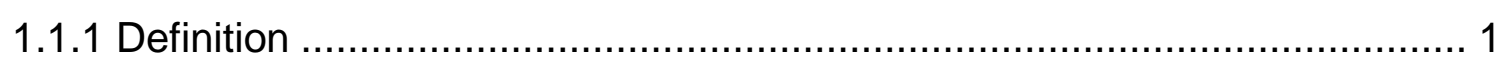

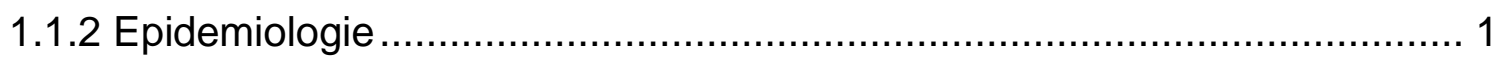

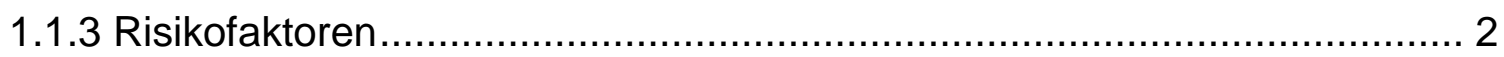

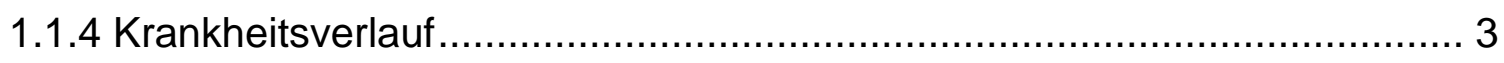

1.1.5 Pathophysiologie und Komorbiditäten .................................................. 4

1.1.5.1 Intrapulmonale Veränderungen bei COPD ....................................... 6

1.1.5.2 Extrapulmonale Effekte der COPD ................................................ 6

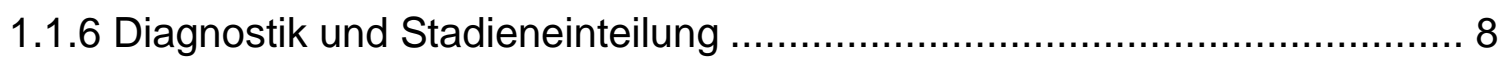

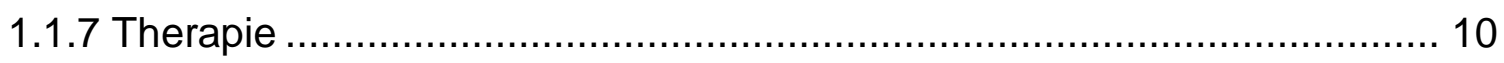

1.2 Das vegetative Nervensystem ............................................................ 11

1.3 Zusammenhang von Herzinsuffizienz und COPD mit dem vegetativen

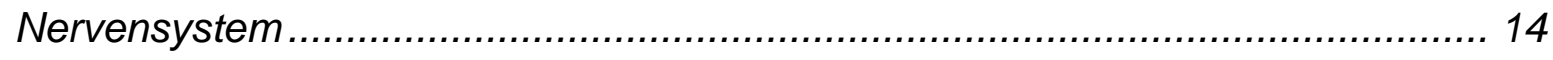

1.5 Untersuchung der sympatho-vagalen Balance ………............................... 16

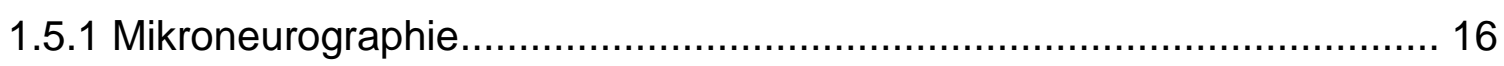

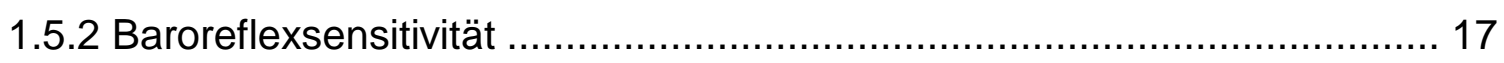

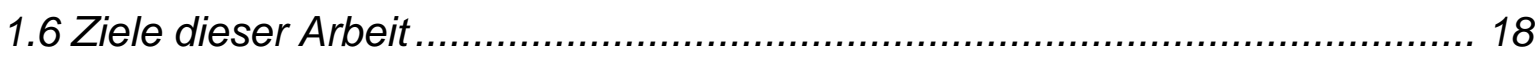

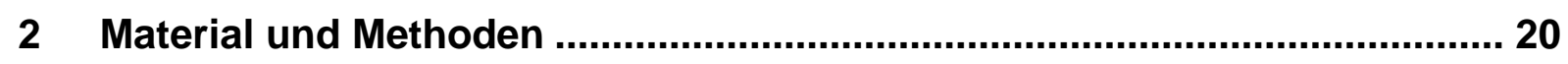

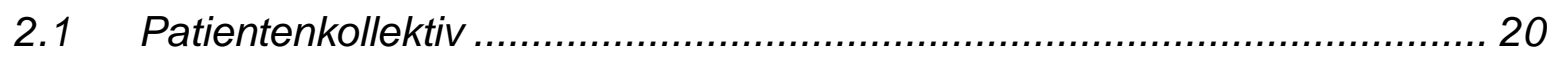

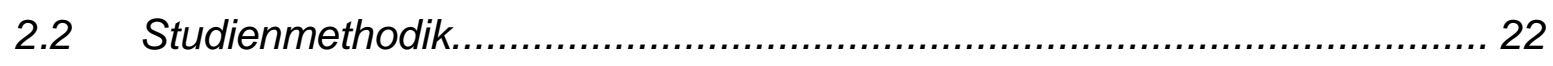

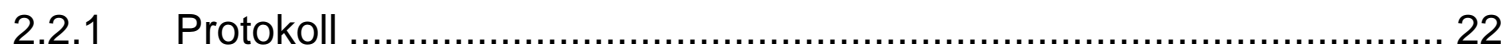

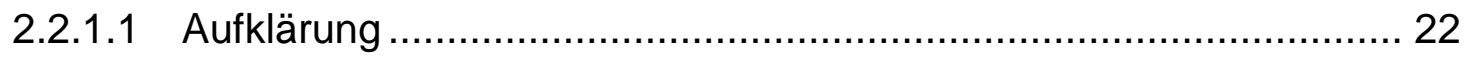

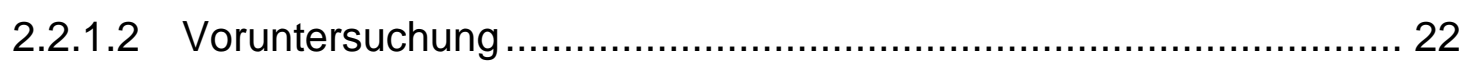

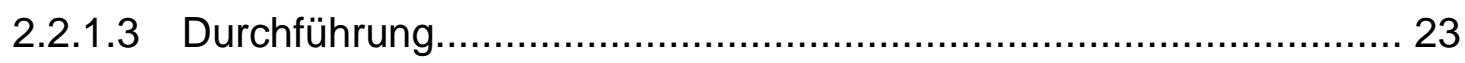

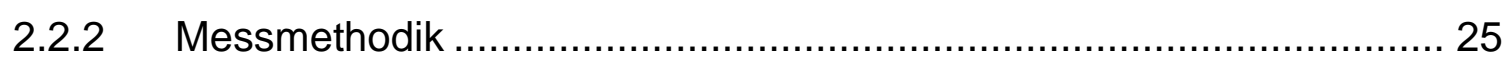

2.2.2.1 Lungenfunktionsuntersuchung ............................................... 25

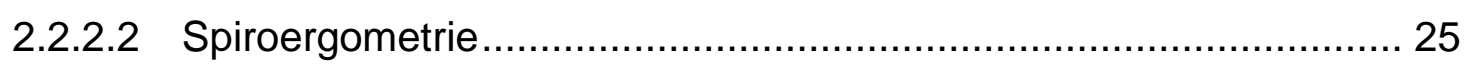

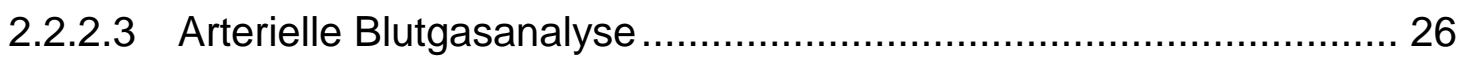


2.2.2.4 Maximale willentliche Kontraktionskraft .................................... 27

2.2.2.5 Kontinuierliche transkutane Blutgasanalyse ............................ 27

2.2.2.6 Herzfrequenz und Blutdruck .............................................. 27

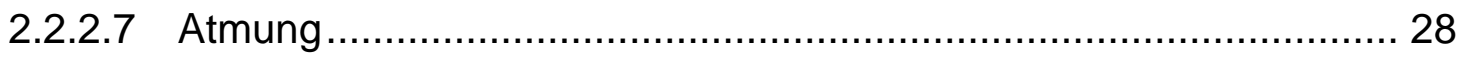

2.2.2.8 Mikroneurographie ......................................................... 29

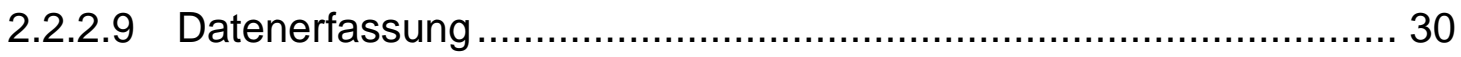

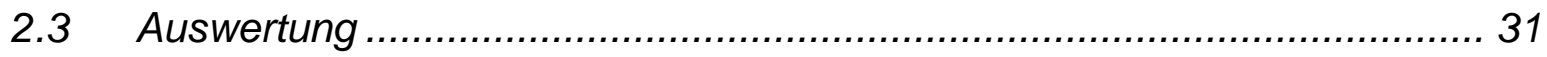

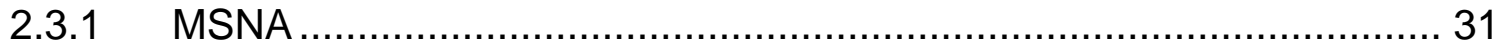

2.3.2 Statistische Methoden ......................................................... 32

2.3.3 Power-Analyse ..................................................................... 32

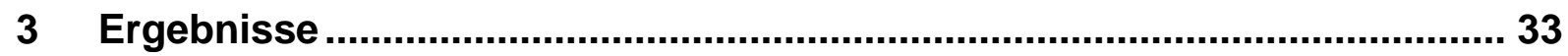

3.1 Teilnehmer-Charakteristika .................................................... 33

3.1.1 Baseline-Charakteristika ................................................... 33

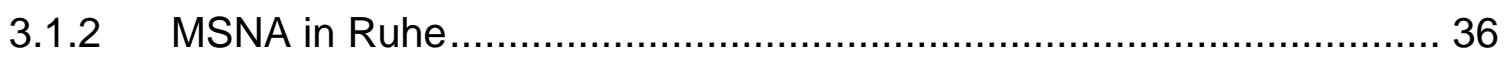

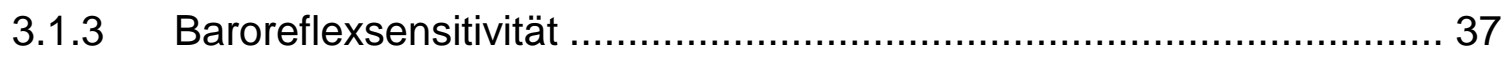

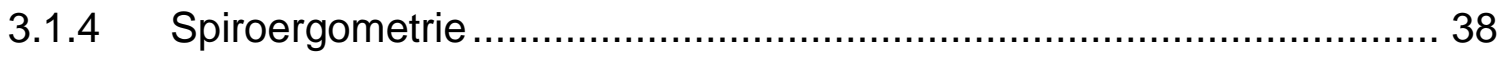

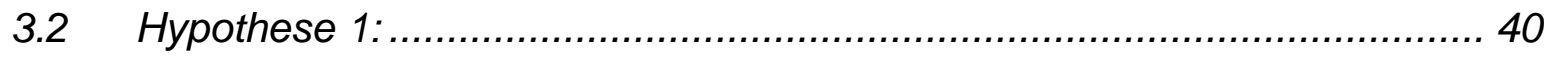

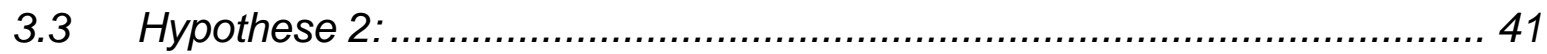

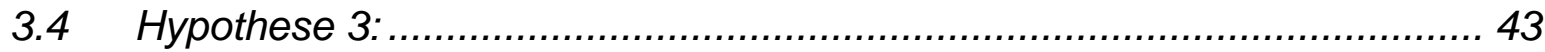

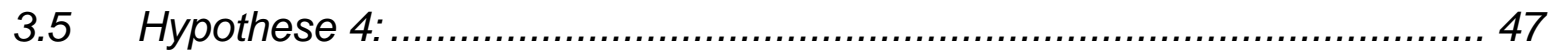

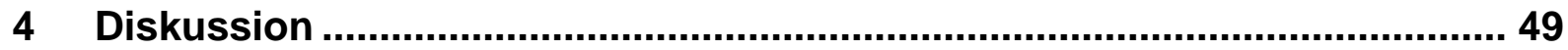

$4.1 \quad$ Teilnehmer-Charakteristika ....................................................... 49

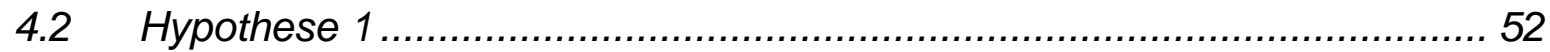

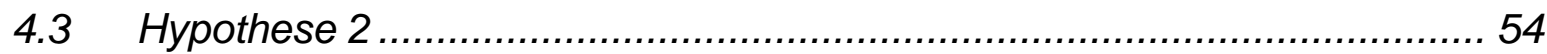

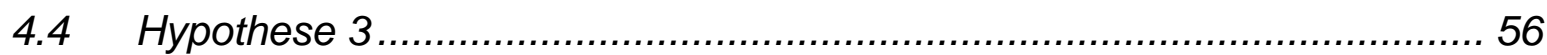

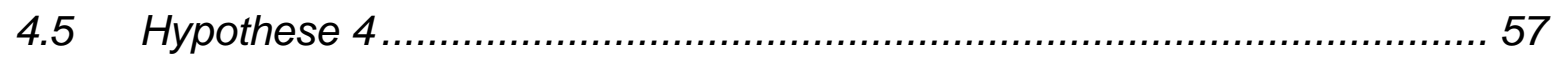

4.6 Stärken- und Schwächenanalyse .............................................. 59

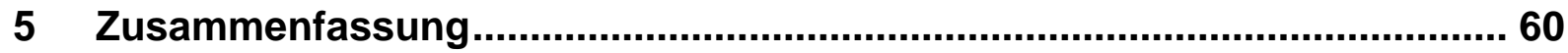




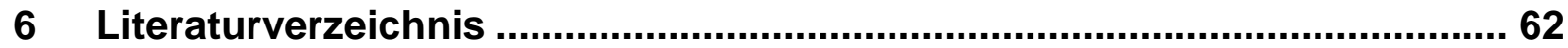

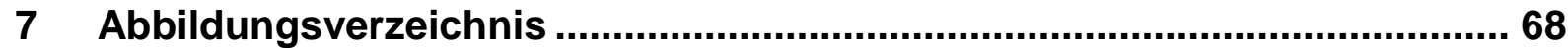

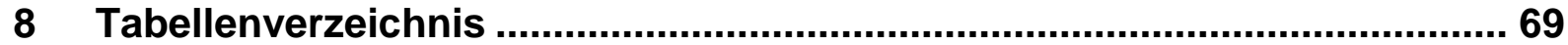




\section{Abkürzungsverzeichnis}

\begin{tabular}{|c|c|}
\hline PW & $\begin{array}{l}\text { arterial pulse wave velocity (arterielle Pulswellen } \\
\text { geschwindigkeit) }\end{array}$ \\
\hline GA & Blutgasanalyse \\
\hline 0 & $\begin{array}{l}\text { level positive airway pressure (biphasische Überdruck- } \\
\text { atmung) }\end{array}$ \\
\hline RS & coreflexsensitivität \\
\hline AT & Assessment Test \\
\hline COPD & $\begin{array}{l}\text { chronic obstructive pulmonary disease (chronisch } \\
\text { obstruktive Lungenerkrankung) }\end{array}$ \\
\hline PAP & $\begin{array}{l}\text { continuous positive airway pressure (kontinuierliche } \\
\text { Überdruckbeatmung) }\end{array}$ \\
\hline $\mathrm{RP}$ & C-reaktives Protein \\
\hline KG & rdiogramm \\
\hline $\begin{array}{l}\mathrm{FEV}_{1} \\
\mathrm{FEV}_{1} / \mathrm{VC}_{\max }\end{array}$ & $\begin{array}{l}\text { exspiratorische Einsekundenkapazität } \\
\text { Einsekundenkapazität }\end{array}$ \\
\hline EVC & forced vital capacity (forcierte Vitalkapazität) \\
\hline $\begin{array}{l}\text { GOLD } \\
\text { mMRC }\end{array}$ & $\begin{array}{l}\text { Global Initiative for Chronic Obstructive Lung Disease } \\
\text { modified British Medical Research Council breathlessness } \\
\text { scale }\end{array}$ \\
\hline SN & xuläre sympathische Nervenaktivität \\
\hline i. & $\begin{array}{l}\text { maximal voluntary contraction (maximale willentliche } \\
\text { Kontraktionskraft }\end{array}$ \\
\hline & nicht-invasive Beatmung \\
\hline & $\begin{array}{l}\text { arterial carbon dioxide partial pressure (arterielle } \\
\text { Kohlenstoffdioxidpartialdruck }\end{array}$ \\
\hline $\mathrm{PaO}_{2}$ & $\begin{array}{l}\text { arterial oxygen partial pressure (arterieller Sauerstoff- } \\
\text { partialdruck) }\end{array}$ \\
\hline EV & spiratory flow (exspiratorische \\
\hline $\mathrm{AW}_{\mathrm{tot}}$ & $\begin{array}{l}\text { lay resistance (totaler Atemwegswiderstand) } \\
\text { rischer Quotient }\end{array}$ \\
\hline RV & nen \\
\hline SEM & $\begin{array}{l}\text { lard error of the mean (Standardfehler des Mitte } \\
\text { s) }\end{array}$ \\
\hline 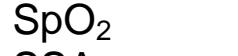 & Courcefoffä̈ttiaung \\
\hline SA & Jautefferenzen) \\
\hline TGV & lumen \\
\hline TLC & (totale Lungenkapazi \\
\hline TNFa & tor $\alpha$ \\
\hline & apazität) \\
\hline & Äquivalent fü \\
\hline
\end{tabular}




\section{$1 \quad$ Einleitung}

\subsection{Die chronisch obstruktive Lungenerkrankung}

\subsubsection{Definition}

Die chronisch obstruktive Lungenerkrankung (COPD) wurde durch die Global Initiative for Chronic Obstructive Lung Disease (GOLD) als Zustand definiert, der durch eine nicht vollständig reversible Einschränkung des Atemflusses gekennzeichnet ist (Pauwels et al. 2001).

Der Begriff COPD umfasst sowohl das Lungenemphysem und die chronische Bronchitis als auch die Erkrankung der kleinen Luftwege. Das Lungenemphysem ist definiert als eine Erweiterung und Destruktion der Lungenalveolen. Die chronische Bronchitis bezeichnet das Vorliegen eines chronischen Hustens mit Sekretverhalt. Nach den Kriterien der Weltgesundheitsorganisation (WHO) müssen Husten und Auswurf in zwei aufeinanderfolgenden Jahren in mindestens drei Folgemonaten bestehen, damit die Bronchitis als chronisch gilt (ATS 1995). Die Erkrankung der kleinen Luftwege bezeichnet die Verengung der kleinen Bronchiolen. Im Unterschied zur chronischen Bronchitis, die reversibel sein kann, setzt die Definition der COPD eine chronische, nicht vollständig reversible Atemwegsobstruktion voraus.

Aufgrund der Relevanz für die Prognose und klinische Einschätzung der COPD wurden auch extrapulmonale Komorbiditäten der COPD wie kardiovaskuläre Erkrankungen, Gewichtsverlust, Osteoporose und muskuläre Dysfunktion in die Definition der GOLD aufgenommen.

\subsubsection{Epidemiologie}

Die chronisch obstruktive Lungenerkrankung ist die gegenwärtig vierthäufigste Todesursache weltweit, wobei bis zum Jahr 2020 mit einem Anstieg auf Platz drei der weltweit häufigsten Todesursachen gerechnet werden muss (Murray und Lopez 1997). In den USA nimmt die COPD bereits seit 2010 Platz 3 der häufigsten Todesursachen ein (Han 2011). 
Aussagen zur Prävalenz in verschiedenen Ländern unterliegen aufgrund unterschiedlicher Definitionen und Diagnosekriterien erheblichen Schwankungen. Die weltweit gemittelte Prävalenz der über 40-Jährigen liegt bei 9-10\%, wobei Raucher wesentlich häufiger als Nichtraucher und Männer häufiger als Frauen betroffen sind (Halbert et al. 2006). Geldmacher et al. ermittelten die Prävalenz der COPD für Deutschland im Rahmen des international durchgeführten Burden of Obstructive Lung Disease-Projektes (BOLD-Studie). Die Auswertung einer repräsentativen Stichprobe von über 40-jährigen Frauen und Männern ergab eine Prävalenz von 13,2\% für das Vorliegen einer COPD. Auch hier waren Raucher und Männer deutlich häufiger betroffen als Nichtraucher und Frauen (Geldmacher et al. 2008). Erschwert werden Aussagen bezüglich der Prävalenz zusätzlich aufgrund der großen Anzahl nicht diagnostizierter Erkrankter.

Die gesundheits- und volkswirtschaftlichen Kosten der COPD sind immens. Nach Hochrechnungen aus Daten der allgemeinen Ortskrankenkassen (AOK) sind die obstruktiven Atemwegserkrankungen COPD und Asthma bronchiale in Deutschland ursächlich für jährlich 25 Millionen Arbeitsunfähigkeitstage, wobei der größte Teil auf die chronische Bronchitis zurückzuführen ist. Die volkswirtschaftlichen Gesamtkosten betragen Schätzungen zufolge 5,93 Milliarden Euro pro Jahr (Konietzko und Fabel 2000).

\subsubsection{Risikofaktoren}

Der Hauptrisikofaktor für die Entwicklung einer COPD ist das Zigarettenrauchen. In longitudinalen Studien konnte eine klare Dosis-Wirkungs-Beziehung zwischen der Stärke des Zigarettenkonsums und der Abnahme der Einsekundenkapazität der Lunge ( $F E V_{1}$ ) belegt werden (Kerstjens et al. 1997). Die Prävalenz der COPD ist bei Männern höher, da in dieser Gruppe auch der Zigarettenkonsum höher ist. Allerdings ist in den letzten 50 Jahren eine Zunahme des Zigarettenkonsums und der Prävalenz der COPD bei Frauen zu beobachten (Pirie et al. 2012). Die Packyears (Zigarettenpackungen/Tag $\times$ Anzahl der Jahre des Zigarettenkonsums) gelten als bester prädiktiver Wert für die Abnahme der Einsekundenkapazität, dennoch besteht eine große Variabilität, die auf weitere Einflussfaktoren zurückzuführen ist. So scheinen Frauen - bei gleicher Exposition - anfälliger für die Entwicklung einer COPD zu sein als Männer (Prescott et al. 1997). Auch das Passivrauchen stellt einen 
signifikanten Risikofaktor für die Entwicklung chronisch respiratorischer Erkrankungen dar. Es besteht eine klare Dosis-Wirkungs-Beziehung zwischen dem Auftreten der chronisch obstruktiven Lungenerkrankung, Lungenkrebs, Hirninfarkten sowie koronarer Herzerkrankungen und der täglichen Rauch-Expositionsdauer ( $\mathrm{He}$ et al. 2012).

Die berufsbedingte Exposition gegenüber bestimmten Stäuben kann Atemwegsbeschwerden und -obstruktionen bedingen und gilt als Risikofaktor für die Entstehung einer COPD. Die Staubexposition führt auch bei Nichtrauchern zu einer Abnahme der Einsekundenkapazität, allerdings ist der Effekt nicht so ausgeprägt wie bei staubexponierten Rauchern. Somit muss die berufsbedingte Staubexposition zwar als Risikofaktor für die Entwicklung einer COPD gesehen werden, allerdings ist inr Stellenwert weitaus geringer als der des Zigarettenkonsums (Morgan 1991). Auch die allgemeine Luftverschmutzung trägt zur Entwicklung einer COPD bei (Chen und Mannino 1999).

Weiter korreliert der sozioökonomische Status in frühen Lebensjahren negativ mit der Entwicklung einer COPD im höheren Alter. Dieser Zusammenhang ist unabhängig vom Rauchverhalten und vom Geschlecht (Prescott et al. 1999).

Ein hereditärer Mangel an $\alpha_{1}$-Antitrypsin gilt als der bedeutendste genetische Risikofaktor für die Entwicklung eines Lungenemphysems (Chappell et al. 2006). Weitere genetische Determinanten für die Ausbildung einer COPD sind Gegenstand aktueller Forschungsprojekte (Pillai et al. 2010).

Frühkindliche Infektionen der unteren Atemwege in den ersten zwei Lebensjahren begünstigen, zusammen mit einem infektbedingten verzögerten Lungenwachstum, die Entwicklung einer COPD im höheren Lebensalter (Shaheen et al. 1994). Als weiterer Risikofaktor für die Entstehung einer COPD gilt ein niedriges Geburtsgewicht. Das mütterliche Rauchverhalten in der Schwangerschaft steht damit in engem Zusammenhang (Juarez und Merlo 2013).

\subsubsection{Krankheitsverlauf}

Normalerweise steigt die Einsekundenkapazität bei Gesunden bis zum frühen Erwachsenenalter kontinuierlich an und nimmt in der zweiten Lebenshälfte langsam 
wieder ab. Die Entwicklung der Lungenfunktion lässt sich durch Umweltfaktoren, insbesondere Zigarettenrauchen, negativ beeinflussen. Für die COPD sind je nach Erkrankungsalter verschiedene Verläufe typisch. Zum einen kann die maximale Einsekundenkapazität im jungen Erwachsenenalter geringer ausfallen als erwartet, sodass die physiologische Abnahme im Alter schneller zu Symptomen führt. Zum anderen kann die Einsekundenkapazität nach Erreichen eines normalen Maximalwertes schneller abfallen. Der Effekt des Zigarettenrauchens auf die Lungenfunktion ist abhängig von der Menge der gerauchten Zigaretten und davon, ob vor abgeschlossener Lungenentwicklung mit dem Rauchen begonnen wurde. Auf der anderen Seite kann die Entwicklung der Lungenfunktion durch Rauchverzicht positiv beeinflusst werden. In der "Lung Health Study" konnte gezeigt werden, dass das Einstellen des Rauchens bei bestehender COPD Grad II einen signifikanten Einfluss auf die Verschlechterung der Einsekundenkapazität hat, der umso größer ist, je mehr Zigaretten zuvor geraucht wurden. Im Vergleich zu COPD-Patienten, die weiterrauchten, verschlechterte sich die Einsekundenkapazität in einem Zeitraum von vier Jahren bei Rauchstopp nur halb so stark (Scanlon et al. 2000). Die jährliche Abnahme der Einsekundenkapazität entspricht nach einem Rauchstopp rasch wieder derjenigen von Nierauchern (Anthonisen et al. 2002).

\subsubsection{Pathophysiologie und Komorbiditäten}

Kennzeichnend für das Vorliegen einer COPD sind eine verminderte relative Einsekundenkapazität ( $\mathrm{FEV}_{1}$ /forcierte Vitalkapazität (FVC); "Tiffeneau-Wert“ $<70 \%$ ), ein vergrößertes Residualvolumen und ein erhöhter Anteil des Residualvolumens (RV) an der totalen Lungenkapazität (TLC) (Pauwels et al. 2001).

In der Spirometrie ist eine Bestimmung der Einsekundenkapazität als Maß der Atemwegsobstruktion möglich. Darüber hinaus ermöglicht die Spirometrie über die Erfassung der Einsekundenkapazität und der forcierten Vitalkapazität (FVC) die Bestimmung des für die Diagnose der COPD maßgeblichen Tiffenau-Wertes. Die relative Einsekundenkapazität ist bei der COPD chronisch erniedrigt. Anders als bei Asthma bronchiale spricht die Atemwegsverengung bei COPD gar nicht oder nur gering auf die Gabe inhalativer Bronchodilatatoren an. Steigerungen der Einsekundenkapazität um mehr als 15\% des Ausgangswertes nach Bronchospasmolyse 
sprechen gegen das Vorliegen einer COPD (Calverley et al. 2003). Die Atemwegsobstruktion imponiert in der Fluss-Volumen-Kurve als charakteristische muldenförmige Veränderung.

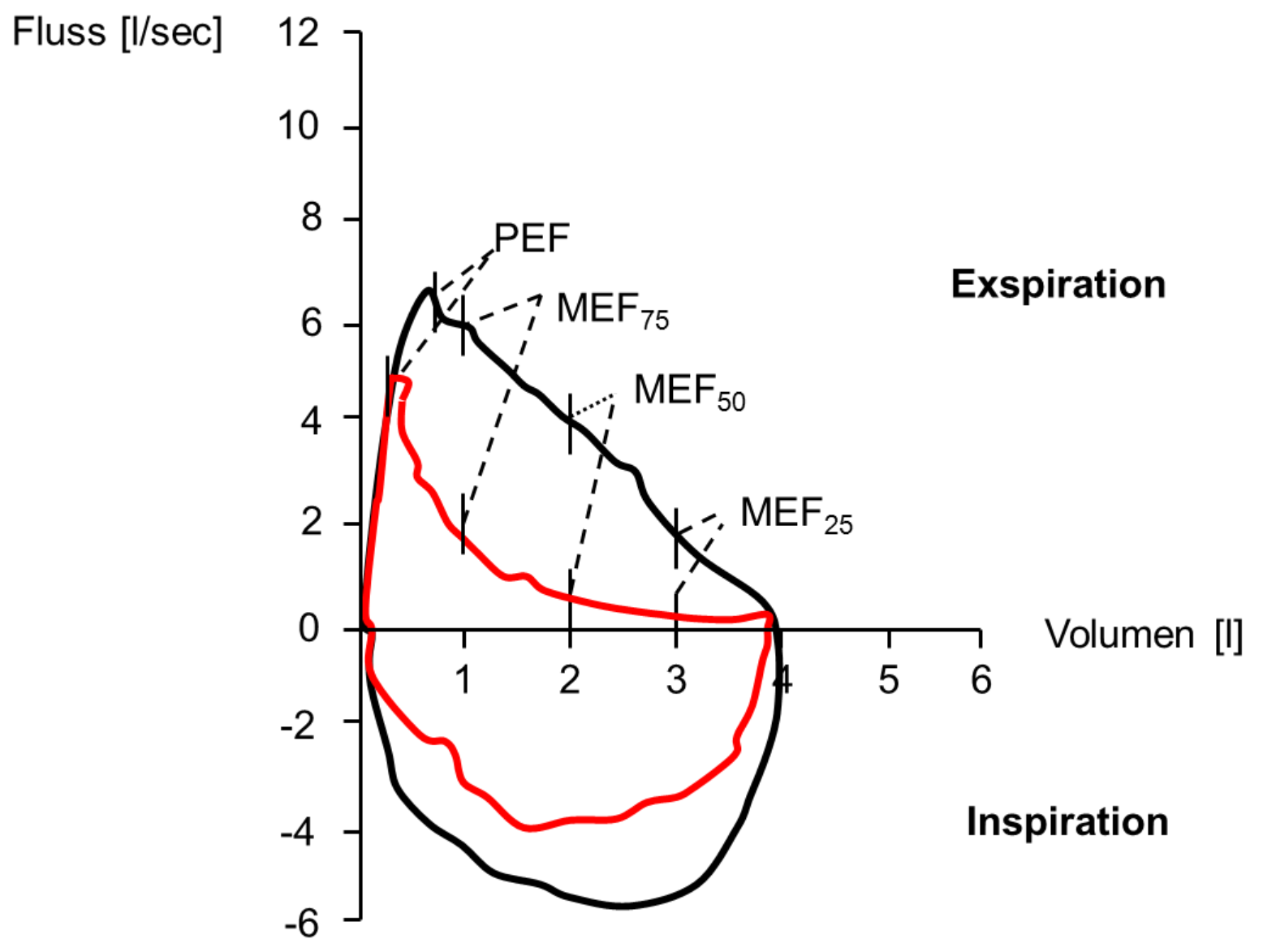

Abbildung 1: Fluss-Volumen-Kurve bei COPD (rot). Die schwarze Kurve stellt die Idealform bei Gesunden dar.

Weiter finden sich bei der COPD häufig ein vergrößertes Residualvolumen und im Verlauf der Erkrankung eine erhöhte TLC als Folge der Überblähung der Lunge. Durch die Überblähung der Lunge kann die Atemwegsobstruktion teilweise ausgeglichen werden, da bei größerem Lungenvolumen stärkere elastische Rückstellkräfte des Lungengewebes wirken und die Exspiration unterstützen. Folge der Überblähung ist aber auch ein Zwerchfelltiefstand, sodass der wichtigste Bestandteil der Atemmuskelpumpe nur noch ineffektiv arbeiten kann. Die Muskelfasern des Zwerchfells sind schon bei maximaler Exspiration verkürzt, sodass 
eine effiziente Kontraktion bei der Inspiration nur mit vergrößerter Muskelspannung möglich ist und so mehr Energie gebraucht wird (O'Donnell 2006), (Cooper 2006).

\subsubsection{Intrapulmonale Veränderungen bei COPD}

Auch das Lungenparenchym zeigt bei Vorliegen einer COPD charakteristische Veränderungen. Die irreversible Zerstörung von Lungenabschnitten distal der Bronchioli terminales führt zur Entstehung pathologisch vergrößerter Lufträume, dem Lungenemphysem. Die vor allem bei Rauchern überproportional hohe Konzentration von Makrophagen und neutrophilen Granulozyten in den Bronchiolen unterstützt das Fortschreiten des Emphysems durch die überschießende Freisetzung von Proteasen zusätzlich. Die Pathophysiologie der COPD ist weiter geprägt durch das Vorliegen einer chronischen systemischen Entzündung, die nicht allein durch das Rauchen erklärt werden kann. Verschiedene Mediatoren werden sezerniert und erhalten die Entzündung aufrecht. Proinflammatorische Zytokine bedingen die Freisetzung von Akut-Phase-Proteinen wie CRP (C-reaktives Protein) und scheinen im Zusammenhang zu stehen mit einer herabgesetzten Muskelkraft (Janssen et al. 2005). Auch der von Makrophagen sezernierte proinflammatorische Tumor-NekroseFaktor a (TNF- $\alpha$ ) ist im Serum von COPD-Patienten erhöht (Di Francia et al. 1994) und mit dem Auftreten von Hypoxie, Kachexie und einer Atrophie der Skelettmuskulatur assoziiert (Tracey et al. 1988). Das vermehrte Auftreten des chemotaktischen Interleukin 8 bedingt eine weitere Rekrutierung von neutrophilen Granulozyten und Monozyten, die wieder mit dem Auftreten einer Muskelschwäche in Verbindung gebracht werden (Spruit et al. 2003). So verstärkt und unterhält sich die chronische Entzündung und hat schließlich eine Zerstörung des Lungengewebes zur Folge. Aus der lokalen Entzündung entwickelt sich im Verlauf eine systemische Entzündung.

\subsubsection{Extrapulmonale Effekte der COPD}

Im Folgenden werden - nach Organen sortiert - einige wesentliche extrapulmonale Veränderungen beschrieben, die im Rahmen einer COPD auftreten können.

- Muskulatur: Die muskuläre Dysfunktion wird neben der chronischen Entzündung auch durch Inaktivität und Mangelernährung unterhalten (Agusti und Soriano 
2008). Des Weiteren ist die Produktion von reaktiven Sauerstoff-Spezies in der Skelettmuskulatur von COPD-Patienten gesteigert, während die Produktion von Antioxidantien vermindert ist. Dem folgt eine weitere Steigerung der MuskelProteolyse und Muskelzell-Apoptose (Barreiro et al. 2005). Zusätzlich bedingen endotheliale Schädigungen eine eingeschränkte Perfusion der Skelettmuskulatur. Der Hauptanteil der Atemarbeit wird durch das Diaphragma verrichtet, das im Gegensatz zur Skelettmuskulatur ständig aktiv ist. Pathologische Veränderungen am Diaphragma stellen daher einen der Hauptgründe für die eingeschränkte körperliche Belastbarkeit von COPD-Patienten dar. Der gesteigerten Atemarbeit und der damit verbundenen Ermüdung des Diaphragmas wirkt der Umbau von Muskelfasern entgegen. Bei Gesunden findet sich im Diaphragma ein Anteil von $39 \pm 2 \%$ weißen Typ-II-Muskelfasern. Bei COPD-Patienten ist der Anteil der Typ-IIMuskelfasern geringer $(29 \pm 3 \%)$, denn die Fasern werden teilweise gegen oxidative Typ-I-Muskelfasern (COPD-Patienten $64 \pm 3 \%$ vs. Kontrolle $45 \pm 2 \%$ ) mit höherer Toleranz gegenüber Erschöpfung ersetzt (Levine et al. 1997).

- Herz-Kreislauf-System: Aufgrund ihrer engen anatomischen Beziehung besteht eine funktionelle und räumliche Kopplung zwischen Herz-Kreislauf-System und Lunge. Pathologische Veränderungen in dem einen System beeinflussen auch direkt das andere. So stellt die Einsekundenkapazität einen unabhängigen Prognosefaktor für das Risiko, an einem Myokardinfarkt zu versterben, dar (Anthonisen et al. 2005). Weiter begünstigt die chronische systemische Entzündung bei COPD-Patienten die Entstehung von arteriosklerotischen Plaques (Agusti und Soriano 2008). Die Komorbidität von Herz-KreislaufErkrankungen und COPD wird außerdem durch gemeinsame Risikofaktoren wie Rauchen, höheres Lebensalter und körperliche Inaktivität unterstützt.

Unter körperlicher Belastung entstehen bei COPD-Patienten hohe intrathorakale Drücke (Montes de Oca und Celli 2000). Die unter Belastung physiologische Steigerung des Herz-Zeit-Volumens ist bei der COPD durch diese hohen Umgebungsdrücke beeinträchtigt. Außerdem findet sich bei COPD-Erkrankten eine erhöhte arterielle Gefäßsteifigkeit (arterial stiffness) (Butler et al. 1988), die einen Risikofaktor für die Entstehung von arterieller Hypertonie und kardiovaskulären Erkrankungen darstellt (Mills et al. 2008).

- Skelettsystem: Vor allem bei Patienten mit höhergradiger COPD (Stadium IV) besteht eine Prävalenz von bis zu 75\% für das Vorliegen von Osteoporose 
(Vrieze et al. 2007). Dies kann zum einen durch ähnliche Risikofaktoren wie höheres Lebensalter, eingeschränkte Mobilität, Rauchen, Mangelernährung oder die Anwendung inhalativer und oraler Glukokortikoide erklärt werden. Zum anderen könnte auch die chronische systemische Entzündung bei der COPD die Entstehung von Osteoporose begünstigen.

\subsubsection{Diagnostik und Stadieneinteilung}

Anamnestisch imponieren bei der COPD Husten, Auswurf und Belastungsdyspnoe mit oft schon lange vor dem ersten Arztkontakt bestehender Symptomatik. Trotz progredienter Entwicklung der Symptome assoziieren die betroffenen Patienten den Erkrankungsbeginn oft mit der ersten Exazerbation. Die Exazerbation bezeichnet das akute, schubförmige Fortschreiten der Erkrankung mit deutlicher Zunahme der Dyspnoe, vermehrtem Husten und Auswurf innerhalb eines kurzen Zeitraumes. Im weiteren Verlauf der Erkrankung kommt es zunehmend zu Einschränkungen durch die Belastungsdyspnoe. Des Weiteren findet im Verlauf der Erkrankung zumeist eine Akzeleration der Exazerbationen statt (Siafakas et al. 1995).

Die Diagnose der COPD wird endgültig durch entsprechende Werte in der Lungenfunktion gesichert. Ausdruck der Atemwegsobstruktion ist eine verminderte Einsekundenkapazität und ein verminderter Tiffenau-Index (s.o.). Im Verlauf der Erkrankung kommt es weiter zu einem Anstieg des Residualvolumens (RV) und der TLC. Da das Residualvolumen verhältnismäßig stärker ansteigt als die totale Lungenkapazität, steigt auch der Quotient RV/TLC, der ein Maß für die Überblähung der Lunge darstellt (Pellegrino et al. 2005). Die Global Initiative for Chronic Obstructive Lung Disease (GOLD) klassifiziert die COPD auf Basis der Spirometrie, klinischer Symptome, dem Exazerbationsrisiko und dem Vorliegen von Komorbiditäten in 4 Gruppen (A-D).

Die Atemwegsobstruktion wird in 4 Grade (GOLD I-IV) unterteilt und orientiert sich an der FEV ${ }_{1}\left(\%\right.$ vom Soll) sowie der FEV ${ }_{1} / \mathrm{VC}$ (siehe Tabelle 1 ). 


\begin{tabular}{|c|c|c|}
\hline Stadium & Einschätzung & FEV $_{\mathbf{1}}$ v. Soll \\
\hline GOLD I & leichtgradig & $\geq 80 \%$ \\
\hline GOLD II & mittelgradig & $50-80 \%$ \\
\hline GOLD III & schwer & $30-50 \%$ \\
\hline GOLD IV & sehr schwer & $<30 \%$ \\
\hline \multicolumn{2}{|c|}{ gültig für Patienten mit FEV $/ \mathrm{VC}<0,7$} \\
\hline
\end{tabular}

Tabelle 1: Schweregradeinschätzung der COPD in der Spirometrie (Vestbo et al. 2013)

Die Einschätzung der klinischen Symptome erfolgt mittels standardisierter Fragebögen wie dem COPD Assessment Test (CAT) oder dem Modified British Medical Research Council (mMRC) breathlessness scale.

Das Exazerbationsrisiko lässt sich adäquat am bisherigen Verlauf der Erkrankung und der Exazerbationsfrequenz in der Vergangenheit abschätzen.

Jeder dieser Parameter wird individuell beurteilt und der Patient entsprechend dem individuellen Risiko einer Gruppe zugeordnet (siehe Tabelle 2). Aus dieser Einteilung ergeben sich entsprechende Therapie-Empfehlungen.

\begin{tabular}{|c|c|c|c|c|c|}
\hline Stadium & Beurteilung & Spirometrie & $\begin{array}{c}\text { Exazerbationen } \\
\text { pro Jahr }\end{array}$ & mMRC & CAT \\
\hline A & $\begin{array}{c}\text { geringes Risiko, } \\
\text { wenige Symptome }\end{array}$ & GOLD 1-2 & $\leq 1$ & $0-1$ & $<10$ \\
\hline B & $\begin{array}{c}\text { geringes Risiko, } \\
\text { ausgeprägte } \\
\text { Symptome }\end{array}$ & GOLD 1-2 & $\leq 1$ & $\geq 2$ & $\geq 10$ \\
\hline C & $\begin{array}{c}\text { hohes Risiko, } \\
\text { wenige Symptome }\end{array}$ & GOLD 3-4 & $\geq 2$ & $0-1$ & $<10$ \\
\hline D & $\begin{array}{c}\text { hohes Risiko, } \\
\text { ausgeprägte } \\
\text { Symptome }\end{array}$ & GOLD 3-4 & $\geq 2$ & $\geq 2$ & $\geq 10$ \\
\hline
\end{tabular}

Tabelle 2: Stadieneinteilung der COPD (Vestbo et al. 2013) 
Parallel zur Therapie der COPD sollte eine adäquate Therapie der extrapulmonalen Manifestationen erfolgen.

Bei dem Verdacht des Vorliegens einer COPD solte zudem ein konventionelles Röntgenbild des Thorax in zwei Ebenen gemacht werden, um ein eventuell bestehendes Lungenemphysem, ein Bronchialkarzinom oder eine Stauung der Lungengefäße frühzeitig zu erkennen (Thurlbeck und Simon 1978).

\subsubsection{Therapie}

Zur Beeinflussung des Krankheitsverlaufs der COPD stehen präventive Maßnahmen, eine medikamentöse Stufentherapie, Physiotherapie, körperliches Training, Ernährungsberatung, apparative Therapieansätze und bei ausgeprägtem Lungenemphysem operative Maßnahmen zur Verfügung. Allerdings bremst nur die Tabakentwöhnung bei Rauchern den Progress der Erkrankung (Anthonisen et al. 2005), (Gulbas et al. 2012). Die Erfolgsquote steigt hier signifikant durch die Teilnahme an professionell geführten Raucherentwöhnungskursen bei gleichzeitiger Substitution von Nikotin mittels Pflaster oder Kaugummi. Weitere Medikamente wie Bupropion und Vareniclin können die Tabakentwöhnung erleichtern. Studien zeigen, dass Raucher, die unterstützend Vareniclin in der Tabakentwöhnung einnahmen, in den ersten 12 Wochen häufiger rauchkarent blieben als die Vergleichsgruppe mit Placebo (Odds Ratio 8,40, 95\% Konfidenzintervall) (Tashkin et al. 2011). Trotz intensiver psychosozialer und medikamentöser Unterstützung liegt die Erfolgsquote der Tabakentwöhnung aber nur bei 35\% nach einem Jahr und 22\% nach 5 Jahren (Anthonisen et al. 1994).

In den Stadien III und IV der COPD wird, vor allem bei gleichzeitigem Vorliegen einer Hyperkapnie, eine Heimbeatmung mittels biPAP (bi-level positive airway pressure), gegebenenfalls mit Sauerstoffzufuhr, empfohlen (Meecham Jones et al. 1995). Die Entlastung der Atemmuskulatur führt in Kombination mit einer Langzeitsauerstofftherapie zu einer Verbesserung der arteriellen Blutgase am Tag, zu größerer Effizienz des Nachtschlafes sowie zu einer subjektiven Steigerung der Lebensqualität (Meecham Jones et al. 1995). Weiter sollte bei im Intervall bestehender Hypoxämie eine Sauerstofflangzeittherapie durchgeführt werden. Hierbei liegt eine adäquate Therapiedauer bei >15 Stunden täglich (Lee et al. 2013). 
Die Sauerstofflangzeittherapie stellt bisher die einzige Maßnahme zur Behandlung der COPD dar, die bei Patienten mit chronischer Hypoxämie erwiesenermaßen einen positiven Effekt auf die Mortalität hat (Group 1980). Ziel aller Therapieansätze im Intervall ist die Symptomreduktion sowie die Verringerung von Stärke und Häufigkeit der Exazerbationen.

Die medikamentöse Behandlung im Intervall richtet sich nach dem Schweregrad der COPD und ist in Tabelle 3 dargestellt.

\begin{tabular}{|c|c|c|c|c|}
\hline \multirow{2}{*}{$\begin{array}{l}\text { Schwere- } \\
\text { grad }\end{array}$} & \multicolumn{3}{|c|}{ Medikamentöse Therapie } & \multirow{2}{*}{$\begin{array}{l}\text { Nicht-medikamentöse } \\
\text { Therapie }\end{array}$} \\
\hline & first line & second line & Alternative & \\
\hline A & $\begin{array}{l}\text { RA } \beta_{2} \text {-Mimetika } \\
\text { / Anticholinergika }\end{array}$ & $\begin{array}{l}\text { LA } \beta_{2} \text {-Mimetika/ } \\
\text { Anticholinergika } \\
\text { oder } \\
\text { RA } \beta_{2} \text {-Mimetika } \\
+ \\
\text { Anticholinergika }\end{array}$ & Theophyllin & $\begin{array}{l}\text { Infektionsprophylaxe: } \\
\text { Impfungen gegen H. } \\
\text { influenzae, } \\
\text { Pneumokokken und } \\
\text { Influenza }\end{array}$ \\
\hline B & $\begin{array}{l}\text { LA } \beta_{2} \text {-Mimetika / } \\
\text { Anticholinergika }\end{array}$ & $\begin{array}{l}\text { LA } \beta_{2} \text {-Mimetika } \\
+ \\
\text { Anticholinergika }\end{array}$ & $\begin{array}{l}\text { RA } \beta_{2} \text {-Mimetika } \\
\text { und/oder } \\
\text { Anticholinergika }\end{array}$ & $\begin{array}{l}\text { Zusätzlich zu A: } \\
\text { Rehabilitationsmaßnah- } \\
\text { men }\end{array}$ \\
\hline C & $\begin{array}{l}\text { Inhalative } \\
\text { Glucocorticoide } \\
+\mathrm{LA} \beta_{2^{-}} \\
\text {Mimetika / } \\
\text { Anticholinergika }\end{array}$ & $\begin{array}{l}\text { LA } \beta_{2} \text {-Mimetika } \\
+ \\
\text { Anticholinergika }\end{array}$ & $\begin{array}{l}\text { Phosphodi- } \\
\text { esterase-4- } \\
\text { Hemmer oder } \\
\text { RA } \beta_{2} \text {-Mimetika } \\
\text { und/oder } \\
\text { Anticholinergika } \\
\frac{\text { oder }}{\text { Theophyllin }}\end{array}$ & Siehe B \\
\hline D & Siehe C & $\begin{array}{l}\text { First line + } \\
\text { Phosphodiester- } \\
\text { ase-4-Hemmer }\end{array}$ & $\begin{array}{l}\text { Carbocystein / } \\
\text { RA } \beta_{2} \text {-Mimetika } \\
\text { und/oder } \\
\text { Anticholinergika } \\
\text { / Theophyllin }\end{array}$ & $\begin{array}{l}\text { Ggf. im Einzelfall bei } \\
\text { Emphysem } \\
\text { Lungenvolumenreduk- } \\
\text { tions-OP, } \\
\text { Lungentransplantation }\end{array}$ \\
\hline
\end{tabular}

Tabelle 3: Stufenschema der COPD-Therapie im Intervall (Vestbo et al. 2013)

\subsection{Das vegetative Nervensystem}

Die Aktivität des vegetativen Nervensystems nimmt eine zentrale Stellung in der Modulation des Herz-Kreislauf-Systems ein. Das vegetative Nervensystem setzt sich aus zwei miteinander interagierenden Teilsystemen zusammen, dem sympathischen und dem parasympathischen Nervensystem. Ein dritter Teil, das enterische 
Nervensystem, spielt in der Steuerung des Herz-Kreislauf-Systems eine untergeordnete Rolle. Die Zellen des vegetativen Nervensystems sind in Hirnstamm und Rückenmark lokalisiert und innervieren mit ihren Zellfortsätzen durch Verschaltung auf weitere Zellen die gesamte glatte Muskulatur und das Myokard. Die meisten Organe werden sowohl vom Parasympathikus als auch vom Sympathikus innerviert, wobei der Parasympathikus in Ruhe überwiegt. Ein gesteigerter Sympathikotonus hingegen bedingt eine Aktivierung des Kreislaufes, ursprünglich im Sinne einer Flucht- oder Kampfsituation. Der Einfluss des Sympathikus ist vor allem durch einen Anstieg der Herzfrequenz, eine Steigerung der kardialen Kontraktionskraft und eine Bronchodilatation gekennzeichnet. Gleichzeitig findet durch Kontraktion kleiner Arterien eine verminderte Durchblutung des Magen-DarmTraktes statt, sodass der Skelettmuskulatur ein größerer Anteil am Herz-ZeitVolumen zur Verfügung steht. Die Neurone des Sympathikus liegen im gesamten thorakalen und oberen lumbalen Abschnitt des Rückenmarks und schließen sich in ihrem Verlauf in der Peripherie den Spinalnerven an.

Der Parasympathikus wird hauptsächlich durch den Nervus vagus, den X. Hirnnerven repräsentiert. Der Nervus vagus tritt im Bereich der Medulla oblongata aus dem Gehirn aus und zieht in seinem Verlauf als eigenständiger Nerv bis in das Abdomen. Ausdruck des Überwiegens des Parasympathikus ist eine Abnahme der Herzfrequenz, eine Konstriktion der Bronchialmuskulatur und eine Steigerung der gastrointestinalen Motorik.

Die regelrechte Interaktion von Sympathikus und Parasympathikus setzt ein Gleichgewicht und eine reziproke Modulation beider Systeme voraus. Entscheidend dafür sind verschiedene Reflexbögen.

Der Baroreflex ermöglicht die schnelle Reaktion des vegetativen Nervensystems auf kurzfristige Änderungen von Blutdruck und Herzfrequenz. Der den Baroreflex steuernde Regelkreis besteht aus intramuralen Pressorezeptoren in der Wand großer intrathorakaler und zervikaler Gefäße, vor allem dem Aortenbogen und den Karotissinus sowie verschiedenen Hirnstammkernen. Die Pressorezeptoren in den Gefäßwänden reagieren auf einen konstanten Dehnungsdruck mit einer Grundfrequenz aus kontinuierlichen Impulsentladungen. Ein steigender Blutdruck bedingt eine erhöhte Summenimpulsfrequenz während ein sinkender Blutdruck die Frequenz der Summenimpulse erniedrigt. Die Pressorezeptoren zeigen außerdem 
ein Proportional-Differential-Verhalten, sodass in der Summenimpulsfrequenz neben der Höhe der Blutdruckänderung auch die Änderungsgeschwindigkeit kodiert wird.

Die Afferenzen der Pressorezeptoren werden über das Rückenmark zum Gehirn geleitet und auf Interneurone der kaudalen ventrolateralen Medulla verschaltet. Ein Teil der Interneurone hemmt sympathische Prämotorneurone während ein weiterer Teil erregend auf präganglionäre parasympathische Kardiomotoneurone wirkt. Bereits bei normalen Blutdrücken werden durch die Grundaktivität der Pressorezeptoren sympathische Efferenzen zum Herz und den Gefäßen gehemmt und gleichzeitig parasympathische Nerven, vor allem der Nervus vagus, zum Herz stimuliert. Eine Senkung des arteriellen Druckes bei erhöhten Werten erfolgt durch eine Abnahme des totalen peripheren Widerstands in den Widerstandsgefäßen sowie einer Zunahme der Kapazität in den Kapazitätsgefäßen. Weiter erfolgt eine Abnahme des Herzzeitvolumens durch negativ chronotrope Effekte des Parasympathikus bei gleichzeitiger Hemmung positiv-inotroper Effekte des Sympathikus. Ist der Blutdruck in Aortenbogen und Karotissinus erniedrigt, laufen entsprechend gegenläufige Prozesse ab (Jänig 2007). Die Stimulation des Baroreflexes durch plötzliche Blutdruckveränderungen in den Glomera aortica und carotica spiegelt sich in einer Änderung der muskulären sympathischen Nervenaktivität (MSNA, siehe auch Kapitel 1.5.1) wider (Wallin et al. 1973).

Der Chemoreflex stellt die Konstanz von Sauerstoff- und KohlenstoffdioxidPartialdruck im arteriellen Blut sicher. Die Partialdrücke werden in den Glomera aortica und carotica sowie in chemosensiblen Arealen des Hirnstamms registriert und im Nucleus tractus solitarii verarbeitet. Bei Hypoxie, Hyperkapnie oder Azidose findet eine Stimulation des Sympathikus und somit eine Steigerung des Herz-ZeitVolumens und des Atemminutenvolumens statt (Spyer 1982).

Der Ergoreflex gewährleistet eine ausreichende Durchblutung der Skelettmuskulatur unter Belastung. Metabolite des Muskelstoffwechsels aktivieren in Kombination mit einer Dehnung der Muskelspindeln das sympathische Nervensystem, das mit einer Steigerung des Herzzeitvolumens und gleichzeitiger Konstriktion peripherer Arterien reagiert. Aus dem so gesteigerten Blutdruck resultiert bei paralleler Azidose- 
bedingter Vasodilatation im arbeitenden Muskel eine Umverteilung der Durchblutung (Crisafulli et al. 2003).

Diese ein sympatho-vagales Gleichgewicht sicherstellenden Reflexbögen unterliegen dem Einfluss des Atemzentrums und des Kreislaufzentrums in der Formatio reticularis (Loewy und Spyer 1990), sodass eine enge wechselseitige Beziehung zwischen vegetativem Nervensystem und kardiorespiratorischem System besteht. Die enge Verknüpfung der Systeme legt die Vermutung nahe, dass kardiorespiratorische Erkrankungen das Gleichgewicht des vegetativen Nervensystems verändern und dass diese Veränderungen sich wiederum auf den Erkrankungsverlauf auswirken könnten.

\subsection{Zusammenhang von Herzinsuffizienz und COPD mit dem vegetativen Nervensystem}

Die Symptomatik der chronisch obstruktiven Lungenerkrankung wird hauptsächlich durch lokale Veränderungen an der Lunge geprägt. Verschiedene Studien haben aber deutlich gemacht, dass auch systemische Einflüsse der COPD den charakteristischen Krankheitsverlauf kennzeichnen (Agusti und Soriano 2008), (Barnes und Celli 2009). Aufgrund des engen Zusammenhangs zwischen der chronischen Entzündung der Atemwege und dem Schweregrad der COPD wurden inflammatorische Prozesse lange als Hauptursache für die systemischen Effekte der COPD verantwortlich gemacht. Neuere Untersuchungen stützen diese These nicht mehr in gleichem Maße. So zeigte die Anwendung des anti-inflammatorisch wirkenden Phosphodiesterase-4-Hemmers Roflumilast bisher keine Effekte auf die Mortalität von COPD-Patienten (Calverley et al. 2007). Nach bisherigen Erkenntnissen profitieren COPD-Patienten auch nicht von einer Behandlung mit dem Tumor-Nekrose-Faktor-a-Antikörper Infliximab, wobei die Einflüsse auf die Entwicklung von Tumoren aufgrund des erst relativ kurzzeitigen Einsatzes noch nicht beurteilt werden können (Rennard et al. 2007). Auch ist noch unklar, wie erhöhte Serumspiegel des CRP (C-reaktives Protein) und die entzündlichen Prozesse an der Lunge zusammenhängen. Es ist aber gesichert, dass der CRP-Serumspiegel keine valide Aussage über die Entwicklung der COPD liefert (de Torres et al. 2008). 
Einige der in Kapitel 1.1.5.2 dargestellten extrapulmonalen Manifestationen der COPD lassen sich auch bei Herzinsuffizienzpatienten nachweisen. Charkoudian und Rabbitts vermuten, dass eine gestörte sympatho-vagale Balance einen Einfluss auf extrakardiale Manifestationen der Herzinsuffizienz hat (Charkoudian und Rabbitts 2009). Passend dazu konnte ein mortalitätssenkender Effekt durch therapeutische Ansätze, die die sympatho-vagale Balance beeinflussen (ß-Blocker, ACE-Hemmer, Mineralocorticoid-Antagonisten) bei Herzinsuffizienzpatienten nachgewiesen werden (Rutten et al. 2010), (Short et al. 2011). Viele Autoren vermuten hinsichtlich der pathophysiologischen Veränderungen am autonomen Nervensystem Parallelen zwischen der COPD und der Herzinsuffizienz. Mortensen et al. konnten auch für den Einsatz von B-Blockern bei COPD-Patienten eine Senkung der Mortalität nachweisen (Mortensen et al. 2009).

Notarius et al. konnten zum einen zeigen, dass bei Herzinsuffizienz-Patienten bereits in Ruhe eine signifikant gesteigerte MSNA vorliegt. Des Weiteren konnte eine unter moderater körperlicher Belastung auftretende Sympathoexzitation beobachtet werden (Notarius et al. 2001). Ähnliches ist auch für die COPD zu erwarten (Heindl et al. 2001), die Einflüsse der COPD auf das vegetative Nervensystem konnten aber noch nicht im Detail erfasst werden.

Eine überschießende Aktivierung des sympathischen Nervensystems äußert sich auch in der Minderung der Baroreflexsensitivität. Die Möglichkeit, auf akute Blutdruckschwankungen adäquat zu reagieren, wird durch die hohe Ruheaktivität des Sympathikus stark eingeschränkt. Bei Herzinsuffizienz-Patienten ist die Baroreflexsensitivität als Ausdruck der bestehenden Sympathoexzitation deutlich vermindert (Osterziel et al. 1995). Der gleiche Effekt lässt sich auch bei COPDPatienten reproduzieren (Raupach et al. 2008).

Weiter beeinflusst die Hypoxie als Ausdruck einer höhergradigen COPD die Aktivität des Sympathikus. Die Effekte der Hypoxie betreffen verschiedene Regelkreise. Zum einen wird eine erhöhte MSNA als Reaktion auf eine Hypoxie-induzierte Vasodilatation im Bereich der Skelettmuskulatur verstanden (Hansen und Sander 2003). Zum anderen können im Plasma von COPD-Patienten mit chronischer Hypoxie eine gesteigerte Renin-Aktivität und eine erhöhte Aldosteron-Konzentration 
gemessen werden (Stewart et al. 1994). Die Aktivitätssteigerung im ReninAngiotensin-Aldosteron-System bedingt über eine erhöhte Vasokonstriktion der kleinen arteriellen Gefäße und eine vermehrte Natrium- und Wasserretention in der Niere einen Blutdruckanstieg. Gleichzeitig wird das Entstehen peripherer Ödeme unterstützt.

Zusätzlich kommt es durch die überschießende Stimulation des Renin-AngiotensinAldosteron-Systems zu dauerhaft erhöhten Angiotensin-II-Serumspiegeln. In hohen Konzentrationen bedingt Angiotensin II neben einer gesteigerten Kollagenproduktion die Unterhaltung oxidativer und inflammatorischer Prozesse sowie die Apoptose von Herz- und Skelettmuskelzellen (Andreas et al. 2005).

Insgesamt besteht ein Zusammenhang zwischen gesteigertem Sympathikotonus und verschiedenen zentralen Regelkreisen des menschlichen Körpers. Trotzdem bestehen zurzeit wenige Therapieoptionen, die MSNA bei COPD-Patienten positiv zu beeinflussen. COPD-Patienten scheinen trotz relativer Kontraindikation aufgrund möglicher Bronchospasmen vom Einsatz von B-Blockern zur Senkung des Sympathikotonus zu profitieren (Rutten et al. 2010). Weitere Methoden, den Sympathikotonus bei COPD-Patienten zu modulieren, sind aktuell Gegenstand der Forschung.

\subsection{Untersuchung der sympatho-vagalen Balance}

Die sympatho-vagale Balance kann mit verschiedenen Methoden untersucht werden; dabei erfassen die experimentellen Verfahren jeweils nur einen Teilaspekt des komplexen Zusammenspiels von Sympathikus und Parasympathikus. Im Folgenden werden zwei häufig genutzte Methoden kurz vorgestellt.

\subsubsection{Mikroneurographie}

Die Mikroneurographie ist eine invasive Methode der direkten Ableitung peripherer Nerven. Sie bietet die Möglichkeit, einzelne myelinisierte oder unmyelinisierte Nervenfasern zu erfassen. Der Nervus peroneus führt neben sensiblen Afferenzen und motorischen Efferenzen zur Skelettmuskulatur auch Efferenzen des vegetativen Nervensystems, welche neben hautsympathischen auch muskelsympathische Anteile besitzen. Die Anteile des vegetativen Nervensystems innervieren hier die 
glatte Muskulatur der Gefäßwand von Blutgefäßen zur Versorgung der Skelettmuskulatur.

Die sympathische Nervenaktivität kann durch die mikroneurographische Ableitung der efferenten muskulären sympathischen Nervenaktivität (MSNA) des Nervus peronaeus superficialis gemessen werden (Delius et al. 1972). Die MSNA ist ein Maß für den Effekt des sympathischen Nervensystems auf das Blutgefäßbett der Skelettmuskulatur und korreliert gut mit dem Plasma-Noradrenalin-Spiegel (Lambert et al. 2011). Die MSNA liefert nicht nur eine bessere Reproduzierbarkeit der sympathischen Nervenaktivität als andere Methoden; mit ihrer Hilfe kann auch eine bessere Aussage über akute und chronische Veränderungen der sympathischen Nervenaktivität getroffen werden (Yucha 2000).

Korrelat der sympathischen Nervenaktivität sind periodisch auftretende Bursts. Die Bursts sind Gruppenentladungen sympathischer Nervenzellen, daher ist die Amplitude der Bursts abhängig von der Anzahl der über die Mikroelektrode abgeleiteten Nervenfasern. Die Bursts treten ausschließlich in der Diastole auf, da der Sympathikus während der Systole durch den Baroreflex gehemmt wird (Hagbarth und Vallbo 1968). Die Anzahl der Bursts pro Zeiteinheit (bursts/min) korreliert mit der Gesamtaktivität des sympathischen Nervensystems

Die Mikroneurographie ist eine Methode, die gut reproduzierbare, valide Ergebnisse liefert. Die Interobserver-Abweichung der Ergebnisse liegt bei 5\%, die IntraobserverAbweichung bei 11\% (Heindl et al. 2001). Es kann aber nur bei etwa $70 \%$ der Probanden ein adäquates Nervensignal abgeleitet werden. Gleichzeitig erfordert die Methode einen großen zeitlichen Aufwand, setzt intakte Efferenzen voraus (z.B. Ausschluss einer diabetischen Polyneuropathie) und ist anfällig für Störvariablen. Aus diesen Gründen hat die direkte Nervenableitung bisher keine Relevanz für die klinische Diagnostik, stellt in der Forschung aber eine häufig verwendete Methode dar.

\subsubsection{Baroreflexsensitivität}

Die Baroreflexsensitivität ist ein $\mathrm{Maß}$ für die Anpassungsgeschwindigkeit der Herzfrequenz auf Blutdruckänderungen und wird in der Einheit $\mathrm{ms} / \mathrm{mmHg}$ angegeben. Die Sensitivität des Parasympathikus wird in der Baroreflexsensitivität widergespiegelt. Eine kontinuierliche Aufzeichnung von EKG und Blutdruck erlaubt 
die nicht-invasive Bestimmung der Baroreflexsensitivität am Computer mittels Spektralanalyse und Alpha-Winkel-Methode. Die Baroreflexsensitivität ist bei Patienten mit höhergradiger COPD aufgrund eines erhöhten pulmonal-arteriellen Druckes und der zentralnervösen Effekte von Hypoxie und Hyperkapnie eingeschränkt (Patakas et al. 1982).

\subsection{Ziele dieser Arbeit}

In früheren Studien der Arbeitsgruppe konnte ein Zusammenhang zwischen der chronisch obstruktiven Lungenerkrankung und einer signifikant erhöhten Aktivität des sympathischen Nervensystems gezeigt werden (Raupach et al. 2008). Weitere Studien verdeutlichen den negativen Einfluss der Erschöpfung der Atemmuskulatur auf die Skelettmuskulatur (Shrikrishna et al. 2012). Die vorliegende Arbeit soll dazu beitragen, den Zusammenhang zwischen der eingeschränkten körperlichen Belastbarkeit, der Dyspnoe und der sympatho-vagalen Imbalance bei COPDPatienten besser zu verstehen. Das Hauptaugenmerk soll dabei auf einem möglichen Zusammenhang zwischen gestörter sympatho-vagaler Balance und der reduzierten körperlichen Belastbarkeit liegen, wie er bereits bei Patienten mit Herzinsuffizienz nachgewiesen werden konnte (Notarius et al. 2001). Sollte eine Korrelation zwischen der gesteigerten Sympathikusaktivität und der eingeschränkten körperlichen Belastbarkeit bei COPD-Patienten bestehen und eine leichte körperliche Belastung eine überproportionale Sympathoexzitation hervorrufen, könnte eine medikamentöse Beeinflussung des vegetativen Nervensystems in Zukunft eine mögliche therapeutische Option zur Therapie der COPD darstellen. Weiter soll untersucht werden, ob der Einsatz von nicht-invasiver Beatmung und die daraus resultierende Verminderung der Atemarbeit bei höhergradiger COPD einen Einfluss auf die MSNA hat. Dies würde wesentlich zum physiologischen Verständnis der positiven Effekte dieses therapeutischen Ansatzes beitragen. Folgende Hypothesen sind zu untersuchen: 
- Die MSNA in Ruhe korreliert bei Gesunden und COPD-Patienten mit dem ventilatorischen Äquivalent für $\mathrm{CO}_{2}\left(\mathrm{VE} / \mathrm{VCO}_{2}\right)$, der maximalen $\mathrm{O}_{2}$-Aufnahme und der körperlichen Belastbarkeit während der Spiroergometrie auf dem Standfahrrad.

- Die Steigerung der MSNA und die Abnahme der Baroreflexsensitivität während einer Handkontraktionsübung als Äquivalent leichter körperlicher Belastung sind bei COPD-Patienten größer als bei gesunden Probanden.

- Die Steigerung der MSNA und die Abnahme der Baroreflexsensitivität während der Handkontraktionsübungen korrelieren mit den in der Spiroergometrie erhobenen Daten.

- COPD-Patienten weisen nach der Benutzung nicht-invasiver Beatmung eine niedrigere MSNA und eine höhere Baroreflexsensitivität in Ruhe und unter leichter körperlicher Belastung auf als vor der Benutzung.

Die Spiroergometrie auf dem Standfahrrad dient der Quantifizierung von Dyspnoe, ventilatorischer Anpassung und Grenze der körperlichen Leistungsfähigkeit unter Belastung. Die Handkontraktionsübungen dienen der Evaluation der ErgoreflexAntwort der Skelettmuskulatur. Die nicht-invasive Beatmung beeinflusst die Ergoreflex-Antwort der Atemmuskulatur in Ruhe und unter Belastung durch eine Entlastung der Atemmuskulatur. 


\section{Material und Methoden}

\subsection{Patientenkollektiv}

In diese dreiarmige Studie wurden Patienten mit chronisch obstruktiver Lungenerkrankung (COPD), aber ohne nicht-invasive Beatmung, Patienten mit COPD und nicht-invasiver Beatmung (NIV) und gesunde Probanden aufgenommen. Bei der Rekrutierung wurde darauf geachtet, dass COPD-Patienten und Kontrollprobanden bezüglich Lebensalter, Körpergröße, Körpergewicht und Geschlecht gematcht wurden. Die Rekrutierung der Patienten erfolgte über die Lungenfachkliniken Immenhausen und Lenglern, über niedergelassene Fachärzte für Lungenheilkunde in Göttingen (Dres. Hinz/Ulbrich, Göttingen; Dres. Ehlers/Schild, Göttingen) und Northeim (Dr. Flade, Northeim) sowie über eine Zeitungsanzeige in der Göttinger Wochenzeitung „Blick“. Die Rekrutierung der gesunden Probanden erfolgte über bestehende Kontakte aus vorangegangenen Studien und ebenfalls eine Zeitungsanzeige. Die Patienten erhielten eine Aufwandsentschädigung von 10 Euro pro Stunde, die Probanden erhielten 15 Euro pro Stunde.

Die Probanden wurden den COPD-Patienten ohne NIV nach Lebensalter, Körpergröße, Körpergewicht und Geschlecht zugeordnet.

Die COPD-Patienten mussten folgende Einschlusskriterien erfüllen:

- Es musste die Diagnose einer COPD im Stadium II oder III nach GOLD durch einen Pneumologen gestellt worden sein.

- Die forcierte exspiratorische Einsekundenkapazität $\mathrm{FEV}_{1}$ musste $<60 \%$ des Solls sein.

- Es musste eine Überblähung der Lunge vorliegen (RV/TLC > 45\%).

- Eine leitliniengerechte medikamentöse Therapie der COPD musste seit mindestens zwei Wochen bestehen.

- Es durfte in den vergangenen drei Wochen vor der Untersuchung keine Exazerbation der COPD stattgefunden haben.

- Im EKG mussten die Patienten einen stabilen Sinusrhythmus aufweisen. 
- COPD-Patienten mit nicht-invasiver Beatmung mussten die Beatmung seit mindestens einem Monat nutzen und mit der Benutzung vertraut sein.

Folgende Kriterien schlossen sowohl COPD-Patienten als auch Probanden von der Studie aus:

- Eine fehlende Einwilligung zur Teilnahme an der Studie

- Patienten, die jünger als 30 Jahre oder älter als 80 Jahre waren

- Patienten und Probanden mit einer in der arteriellen Blutgasanalyse bestätigten Hypoxämie $\left(\mathrm{PaO}_{2}<55 \mathrm{mmHg}\right)$ oder Hyperkapnie $\left(\mathrm{PaCO}_{2}>45\right.$ mmHg bzw. für COPD-Patienten mit NIV $\mathrm{PaCO}_{2}>55 \mathrm{mmHg}$ )

- Die systemische Anwendung von Medikamenten mit sympathomimetischer Wirkkomponente (z. B. Theophillin, Moxonidin, Clonidin)

- Die orale Einnahme kurzwirksamer $\beta_{2}$-Sympathomimetika

- Die bestehende Diagnose eines obstruktiven Schlafapnoe-Syndroms

- Die Diagnose einer eingeschränkten linksventrikulären Pumpfunktion (EF < $60 \%)$

- Echokardiographische Hinweise auf eine systolische oder diastolische Dysfunktion, eine pulmonal-arterielle Hypertonie oder ein höhergradiges Vitium $\left(>1^{\circ}\right)$

- Die Diagnose einer primär pulmonal-arteriellen Hypertonie

- Ein stattgehabter Myokardinfarkt, eine perkutane transluminale CoronarAngioplastie oder eine Aorto-coronare venöse Bypass-Operation in den vorangegangenen zwei Monaten

- Eine klinisch apparente Polyneuropathie

- Eine medikamentös behandelte Diabetes mellitus-Erkrankung

- Andere schwere (lebensbedrohliche) Begleiterkrankungen, inkl. Malignome 


\subsection{Studienmethodik}

\subsubsection{Protokoll}

Die Studie fand in einem Zeitraum von 13 Monaten von Juli 2011 bis Juni 2012 in der Universitätsmedizin Göttingen statt. Jeder Patient/Proband wurde zu einem einmaligen, etwa siebenstündigen Termin in die Universitätsmedizin Göttingen einbestellt, an dem die Patientenaufklärung, die Voruntersuchung, die pneumologische und kardiologische Funktionsdiagnostik sowie die klinisch-experimentellen Versuche durchgeführt wurden.

Alle Patienten und gesunden Probanden wurden vor der Untersuchung über das Vorgehen aufgeklärt und erklärten schriftlich ihr Einverständnis zur Teilnahme an der Studie. Das Studienprotokoll wurde der Ethikkommission der medizinischen Fakultät der Georg-August-Universität Göttingen vorgelegt (Antragsnummer 11/10/08). Diese stellte in ihrem Bescheid vom 4.11.2008 fest, dass keine ethischen oder rechtlichen Einwände gegen das Studienvorhaben bestanden.

Die Durchführung der Studie wurde von der Deutschen Forschungsgemeinschaft unterstützt (Projekt RA 1937/1-1).

\subsubsection{Aufklärung}

Die Patienten/Probanden erhielten vorab detaillierte schriftliche Informationen über den Zweck und das Ausmaß der Studie sowie den eigentlichen Versuchsablauf. Des Weiteren wurden alle Teilnehmer an der Studie auf eventuelle Risiken, vor allem auf mögliche Schädigungen des Nervus peronaeus durch die Mikroneurographie, hingewiesen. Die Patienten/Probanden wurden aufgefordert, sich bei eventuell auftretenden Nachwirkungen der Untersuchung, insbesondere Missempfindungen, unverzüglich mit dem Studienarzt in Verbindung zu setzen. Das Aufklärungsgespräch wurde mit einer schriftlichen Einverständniserklärung dokumentiert.

\subsubsection{Voruntersuchung}

Vor Aufnahme in die Studie erfolgte bei jedem Patienten/Probanden eine umfassende Voruntersuchung, um eventuelle Ausschlusskriterien bzw. fehlende Einschlusskriterien zu erkennen. Die Patienten/Probanden wurden vom Studienarzt körperlich untersucht und sowohl die Lunge als auch das Herz auskultiert. Weiter 
wurde ein 12-Kanal-Ruhe-Elektrokardiogramm geschrieben, um mögliche Herzrhythmusstörungen, insbesondere ein Vorhofflimmern, zu erkennen. Eine Bodyplethysmographie wurde zur Bestimmung der respiratorischen Parameter $\left(F_{1} V_{1}\right.$, VCin-B, FEV 1 NCmax, RV/TLC-B) durchgeführt. Bei den COPD-Patienten wurde durch eine transthorakale Echokardiographie und eine Dopplerechokardiographie das Vorliegen von höhergradigen Vitien, einer systolischen oder diastolischen Dysfunktion oder anderen strukturellen Veränderungen des Herzens ausge-schlossen. Die Probanden erhielten keine transthorakale Echokardiographie/Dopplerechokardiographie. Die transthorakale Echokardiographie/ Dopplerechokardiographie wurde durch erfahrene Ärztinnen und Ärzte im Herzzentrum der Universitätsmedizin Göttingen durchgeführt. Des Weiteren wurde eine arterielle Blutgasanalyse durchgeführt. Außerdem wurde die maximale willentliche Kontraktionskraft der nicht-dominanten Hand bestimmt und die körperliche Leistungsfähigkeit mittels einer Spiroergometrie gemessen. Zusätzlich wurde die arterielle Pulswellengeschwindigkeit (aPWV) dokumentiert.

\subsubsection{Durchführung}

Die Patienten/Probanden wurden vormittags, zwei Stunden nach der Einnahme eines niedrig-kalorischen Frühstücks, einbestellt. Am Tag der Untersuchung waren die Patienten/Probanden angehalten, weder koffeinhaltige Speisen noch Getränke zu sich zu nehmen. Außerdem war ein Tabakverzicht von mindestens drei Stunden vor der Untersuchung Voraussetzung für die Teilnahme an der Studie. Die Patienten/Probanden wurden gebeten, ihre normale Medikation mit Ausnahme diuretischer Medikamente (mögliche Sympathikus-Aktivierung) am Morgen wie gewohnt einzunehmen.

Nach schriftlicher Einwilligung und erfolgter Voruntersuchung wurden die Patienten/Probanden auf der Untersuchungsliege in Rückenlage mit etwa $30^{\circ}$ erhöhtem Oberkörper gelagert. Anschließend wurden kontinuierliche Blutdruckmessung, Oberarm-Blutdruckmanschette, Pulsoxymetrie sowie Zubehör zur Messung der Atemexkursionen und zur Durchführung der transkutanen Blutgasanalyse angelegt. 
Der Nervus peronaeus wurde zunächst transkutan elektrisch stimuliert und sein Verlauf markiert. Anschließend wurden Referenz- und Messelektrode eingeführt und die Position solange verändert, bis ein adäquates Signal empfangen wurde. Bei so optimiertem Signal wurde mit der Aufzeichnung der Protokolle A-C begonnen. Die Durchführung der Protokolle dauerte insgesamt etwa 90 Minuten.

Protokoll A bestand aus der Aufzeichnung des MSNA-Ruhesignals für 10 Minuten. Der Patient/Proband wurde aufgefordert, sich für diesen Versuchsteil vollständig zu entspannen. Das Protokoll A diente sowohl als Maß für die Grundaktivität des sympathischen Nervensystems bei dem entsprechenden Patienten/Probanden als auch als Referenzwert für spätere Messwerte. Außerdem sollten mögliche Unterschiede zwischen Patienten und Probanden dokumentiert werden. Die so erhobene Grundaktivität des sympathischen Nervensystems wurde später mit dem Grad der Dyspnoe, dem ventilatorischen Äquivalent für $\mathrm{CO}_{2}\left(\mathrm{VE} / \mathrm{VCO}_{2}\right)$ und der Limitierung der körperlichen Leistungsfähigkeit in der Spiroergometrie korreliert.

Das Protokoll B bestand aus zwei verschiedenen Teilen, deren Reihenfolge randomisiert wurde. In einem Versuchsteil sollten die Patienten/Probanden zwei Minuten lang eine isometrische Kontraktion der nicht-dominanten Hand mit $30 \%$ ihrer maximalen willentlichen Kontraktionskraft (MVC) halten. Hierzu wurde ein Handdynamometer (Handgrip dynamometer model 78010, Lafayette Instrument, Loughborough, Leicester, United Kingdom) verwendet und die aufgebrachte Kraft durch den Versuchsleiter überwacht. Im anderen Versuchsteil sollten für wiederum zwei Minuten rhythmische Handkontraktionen mit $50 \%$ der MVC ausgeführt werden. Der Schweregrad der Dyspnoe während der Belastung wurde vom Patienten anhand einer Borg-Skala angegeben. Nach jedem Versuchsteil wurde für jeweils 10 Minuten die Ruheaktivität aufgezeichnet, sodass Herzfrequenz und Blutdruck wieder das Ausgangsniveau erreichten.

Protokoll $C$ bestand für Probanden und COPD-Patienten ohne NIV in einer Wiederholung der Protokolle A und B. COPD-Patienten mit NIV wurden für 20 Minuten an das eigene, mitgebrachte biPAP-Beatmungsgerät angeschlossen und beatmet. Die gewählten Beatmungsparameter entsprachen denen, die dem 
Patienten durch den behandelnden Pneumologen vorgegeben waren. Nach Aufzeichnung der 20 Minuten Ruheaktivität unter NIV wurden die Patienten vom biPAP-Gerät getrennt, und es wurde erneut das Protokoll B durchgeführt.

\subsubsection{Messmethodik}

\subsubsection{Lungenfunktionsuntersuchung}

Im Rahmen der Eingangsuntersuchung wurde bei Patienten und Probanden eine Lungenfunktionsuntersuchung mittels einer Ganzkörperplethysmographie (Ganshorn PowerCube LF8.4F, Ganshorn Medizin Electronic, Niederlauer, Germany) nach den Vorgaben der European Respiratory Society durchgeführt. Der Patient sitzt dazu in einer luftdicht verschlossenen Kabine mit etwa 1000 Liter Volumen und atmet über ein Mundstück außerhalb der Kabine befindliche Luft. Durch die Atemexkursionen des Thorax und Abdomen entstehen Druckschwankungen im Inneren der Kabine, aus denen ein Computer das intrathorakale Gasvolumen und verschiedene andere respiratorische Parameter berechnen kann. Gleichzeitig können am Ganzkörperplethysmographen eine Spirometrie durchgeführt und weitere respiratorische Parameter erhoben werden.

Folgende Werte wurden im Rahmen der Lungenfunktionsuntersuchung erhoben:

- Vitalkapazität (VC in \% v. Soll)

- Forcierte exspiratorische Einsekundenkapazität $\left(\mathrm{FEV}_{1}\right.$ in I)

- Relative Einsekundenkapazität ( $\mathrm{FEV}_{1} / \mathrm{VCmax}$ in \%)

- Exspiratorischer Spitzenfluss (PEF in I/s)

- Totaler Atemwegswiderstand (RAWtot in kPas/l)

- Residualvolumen (RV in I)

- Totale Lungenkapazität (TLC in I)

Des Weiteren wurden die Resistance, Fluss/Volumen, Volumen/Zeit, Thorakales Gasvolumen(TGV) und Spirometrie-Body grafisch dargestellt.

\subsubsection{Spiroergometrie}

Die Spiroergometrie ist ein Verfahren, bei dem die Leistungsfähigkeit des kardiopulmonalen Systems ermittelt werden kann. Der Patient tritt auf einem Standfahrrad gegen einen variablen Widerstand bis zur völligen Erschöpfung an. 
Die Leistung wurde bei Probanden alle 2 Minuten um 20 Watt bis zur vollständigen Erschöpfung gesteigert. Bei COPD-Patienten wurde das Protokoll, falls nötig, auf die individuelle körperliche Belastbarkeit angepasst und die Leistung alle 2 Minuten um 5 Watt gesteigert, um eine adäquate Messung durchführen zu können. Abbruchkriterien waren eine Senkung der ST-Strecke im EKG um $>3 \mathrm{~mm}$, ein Blutdruckabfall um $>10 \mathrm{mmHg}$ gegenüber den Ausgangswerten, pectanginöse Beschwerden, subjektiv maximale Luftnot oder völlige Erschöpfung.

Es wurden folgende Werte erhoben:

- maximale Sauerstoffaufnahme $\left(\mathrm{VO}_{2}\right.$ in $\left.1 / \mathrm{min}\right)$

- maximale Kohlenstoffdioxidabgabe $\left(\mathrm{VCO}_{2}\right.$ in $\left.\mathrm{l} / \mathrm{min}\right)$

- Respiratorischer Quotient $\left(\mathrm{RQ}=\mathrm{VO}_{2} / \mathrm{VCO}_{2}\right)$

- Ventilatorisches Äquivalent für Sauerstoff $\left(\mathrm{VE} / \mathrm{VO}_{2}\right)$

- Ventilatorisches Äquivalent für Kohlenstoffdioxid $\left(\mathrm{VE} / \mathrm{VCO}_{2}\right)$

- maximale Leistungsfähigkeit [W]

\subsubsection{Arterielle Blutgasanalyse}

Zur Erhebung des arteriellen Sauerstoffpartialdruckes $\left(\mathrm{PaO}_{2}\right)$ und des arteriellen Kohlenstoffdioxid-Partialdruckes $\left(\mathrm{PaCO}_{2}\right)$ wurde bei den Patienten eine arterielle Blutgasanalyse (BGA) durchgeführt. Zur Gewinnung der arteriellen Blutprobe wurde die Arteria radialis im Bereich des Handgelenks mit einer BGA-Kanüle punktiert und $2 \mathrm{ml}$ arterielles Blut gewonnen. Bei Probanden wurde statt der arteriellen eine kapillare Blutgasanalyse mit einer Blutprobe von $200 \mu \mathrm{l}$ aus dem hyperämisierten Ohrläppchen durchgeführt. Die Analyse der Blutproben erfolgte durch das BGAGerät GEM®) Premier 4000 (GEM®) Premier 4000, Instrumentation Laboratory UK Ltd, Warrington, Cheshire, United Kingdom). Ziel der BGA war der Ausschluss von $\mathrm{PaO}_{2}$-Werten $<55 \mathrm{mmHg}$ und $\mathrm{PaCO}_{2}$-Werten $>45 \mathrm{mmHg}$ bei Probanden und COPD-Patienten ohne NIV bzw. > $55 \mathrm{mmHg}$ bei COPD-Patienten mit NIV (siehe Ausschlusskriterien). Außerdem wurden die erhobenen Werte zur in-vivo-Kalibration der kontinuierlichen transkutanen Blutgasanalyse (siehe 2.2.2.5) benötigt. 


\subsubsection{Maximale willentliche Kontraktionskraft}

Im Rahmen der Eingangsuntersuchung wurde die maximale willentliche Kontraktionskraft (MVC in kg) der Unterarmmuskulatur der nicht-dominanten Hand mittels eines Handdynamometers festgestellt (Handgrip dynamometer model 78010, Lafayette Instrument, Loughborough, Leicester, United Kingdom). Die Patienten/Probanden wurden aufgefordert, in insgesamt drei Versuchen mit maximaler Kraft die Hand zuzudrücken. Die erzeugte Kraft in Kilogramm(kg) wurde auf dem Handdynamometer angezeigt. Der Mittelwert der drei Versuche wurde gebildet und notiert. Im Versuchsteil führten die Patienten/Probanden für jeweils 2 Minuten rhythmische Kraftübungen mit 50\% der MVC bzw. statische Kraftübungen mit $30 \%$ der MVC durch.

\subsubsection{Kontinuierliche transkutane Blutgasanalyse}

Während der gesamten Versuchsdurchführung wurden transkutan die Partialdrücke für Sauerstoff $\left(\mathrm{PaO}_{2}\right)$ und Kohlenstoffdioxid $\left(\mathrm{PaCO}_{2}\right)$ über eine auf dem Thorax des Patienten angebrachte Elektrode gemessen (TCM3-Monitor und TCC-Kalibrierung, Radiometer, Copenhagen, Danmark). Zu Beginn des Versuchs wurde das Gerät anhand der zuvor entnommenen arteriellen BGA kalibriert. Durch die Messung der Blutgase konnte bestätigt werden, dass die Patienten/Probanden zu keinem Zeitpunkt des Versuches hyperkapnisch bzw. hypoxisch waren und somit eine eventuell vorbestehende sympathische Aktivierung ausgeschlossen werden (Morgan et al. 1995).

Die periphere Sauerstoffsättigung $\left(\mathrm{SpO}_{2}\right.$ in \%) wurde photometrisch über einen Fingerclip (Datex® AS/3, Hitachi Hi-Tech Corp., Tokyo, Japan) gemessen.

\subsubsection{Herzfrequenz und Blutdruck}

Die Herzfrequenz der Patienten/Probanden wurde kontinuierlich über ein Elektrokardiogramm (EKG) aufgezeichnet (Datex® AS/3, Hitachi Hi-Tech Corp., Tokyo, Japan). Das EKG wurde über drei nach dem Einthoven-Schema für Extremitätenableitungen auf dem Thorax angebrachte Klebeelektroden abgeleitet. Die kontinuierliche Blutdruckmessung erfolgte automatisch, nicht-invasiv mit dem Portapres $\AA^{\circledR}-$ System (Portapres ${ }^{\circledR}$ device, Finapres Medical Systems BV, Amsterdam, Netherlands). Über zwei an Mittelfinger und Ringfinger angebrachte aufblasbare 
Fingermanschetten kann der Blutdruck pulssynchron im Abstand von zwei Herzschlägen gemessen werden. Die Manschetten erzeugen einen auf die Arteria digitalis palmaris propria ausgeübten Druck, der sich pulssynchron verändert. Mit dem Prinzip der Vascular unloading technique wird aus dem durch die Pulswelle erzeugten Druckunterschied der arterielle Blutdruck ermittelt (Yamakoshi et al. 1980). Die Manschetten lagen in drei verschiedenen Größen vor, sodass für jeden Patienten/Probanden eine ideale Passform gewährleistet war. Aufgrund der kontinuierlichen Blutdruckmessung war die Berechnung der Baroreflexsensitivität möglich. Zusätzlich wurde der Blutdruck automatisch alle 3 Minuten über eine Oberarm-Blutdruckmanschette gemessen (Datex® AS/3, Hitachi Hi-Tech Corp., Tokyo, Japan), um die Werte des Portapres®-Systems zu verifizieren.

\subsubsection{Atmung}

Die Atemfrequenz und das Atemzugvolumen wurden mithilfe der respiratorischen induktiven Plethysmographie (Respitrace ${ }^{\circledR}$ device, Respitrace Systems, Ambulatory Monitoring Inc., Ardsley, New York, USA) gemessen. Bei diesem Verfahren werden dem Patienten/Probanden zwei elastische, individuell auf den Brust- und Bauchumfang angepasste Gurte an Thorax und Abdomen angelegt. Die Gurte werden über je zwei Elektroden mit der Recheneinheit und einem Oszillator verbunden. Bei den Atemexkursionen werden die Gurte und die darin eingewebten Drahtspiralen unterschiedlich stark gedehnt, was sich als eine Veränderung in der Frequenz der Oszillationen darstellt. In der Recheneinheit werden die unterschiedlichen Oszillationsfrequenzen in elektrische Spannung umgerechnet und nach Digitalisierung durch den Analog-Digital-Wandler (siehe 2.2.2.9) auf dem angeschlossenen Notebook dargestellt. Die Berechnung der Atemfrequenz und des Atemzugvolumens erfolgte anhand von Referenzwerten, die bei jedem Patienten/Probanden vorher individuell erhoben wurden. Dazu atmete der Patient/Proband ein definiertes Volumen von $450 \mathrm{ml}$ mit Hilfe eines speziellen Plastikbeutels (Spirobag, Respitrace Systems, Ambulatory Monitoring Inc., Ardsley, New York, USA) mehrfach ein und aus. Dieser Versuch wurde im Stehen und auch im Liegen durchgeführt, sodass die Spannungsänderungen mit dem Atemzugvolumen korreliert werden konnten. 


\subsubsection{Mikroneurographie}

Die Summenaktionspotentiale der muskelsympathischen Fasern bilden das neurophysiologische Korrelat zur muskelsympathischen Nervenaktivität (MSNA). Der Nervus peronaeus superficialis eignet sich aufgrund seiner oberflächlichen Lage gut, um die MSNA zu erfassen. Die über eine Wolfram-Mikroelektrode abgeleiteten Summenaktionspotentiale imponieren im integrierten Signal als Entladungen mit charakteristischem Aussehen, sogenannten Bursts. Die Bursts der MSNA sind charakterisiert durch einen raschen Aufstrich mit nur einer einzigen Spitze. Außerdem äußert sich sowohl die haut- als auch muskelsympathische Nervenaktivität in der akustischen Darstellung als gut zu differenzierendes Signal. (Delius et al. 1972).

Zur besseren Orientierung wurde der Nervus peronaeus superficialis zunächst mittels externer elektrischer Stimulation dargestellt. Dazu wurden über die Elektrode eines elektrischen Nervenstimulationsgerätes (Stimuplex® HNS 11, B Braun, Melsungen, Germany) Stromimpulse mit einer Stromstärke von 1-5 mA und einer Impulsdauer von $1 \mathrm{~ms}$ im Bereich kranial des Caput fibulae abgegeben. Bei Annäherung der Elektrode an den Nerven spürte der Patient/Proband zunächst Kontraktionen der prätibialen Muskulatur, die bei optimierter Position der Elektrode auch visuell vom Untersucher bestätigt werden konnten. Der Verlauf des Nervs wurde mit einem wasserfesten Stift markiert. Dann erfolgte die subkutane Platzierung einer Referenzelektrode im Bereich lateral der Patella. Die Referenzelektrode entsprach vom Aufbau der eigentlichen Mikroneurographie-Elektrode. Anschließend wurde die Wolfram-Mikroelektrode mit einem Schaftdurchmesser von $200 \mu \mathrm{m}$ und einem Spitzendurchmesser von 1-5 $\mu \mathrm{m}$ in den Nerv eingeführt. Das Nervensignal wurde abgeleitet und über den Nerve Traffic Analyzer (The University of lowa, 8 Medical Lab, lowa City, USA) maximal $10^{6}$-fach verstärkt. Das Signal wurde über einen Lautsprecher als Rauschen und parallel über den Analog-Digital-Wandler am angeschlossenen Notebook als Kurve dargestellt. Das Durchstechen der den Nerven umgebenden Faszie imponierte als charakteristischer "Insertion Burst", während die MSNA als periodische Bursts in Erscheinung trat. Die MSNA-Bursts wurden auch visuell als pulssynchrone, in der Frequenz vom Grad der sympathischen Nervenaktivität abhängige Spitzen sichtbar. 
Die MSNA musste anschließend gegenüber sympathischen Hautefferenzen (SSA) verifiziert werden. Dies geschah über drei durchgeführte Manöver:

- Das Beklopfen der prätibialen Muskulatur löste bei korrekter Lage der Elektrode ein zum Klopfen synchrones Rauschen aus.

- Bei versehentlicher Ableitung von sympathischen Hautefferenzen löste das Bestreichen des Fußrückens ein dazu synchrones Rauschen aus, bei Ableitung der MSNA hatte das Bestreichen der Haut keinen Effekt.

- Wurde der Patient/Proband durch den Untersucher erschreckt, löste dies eine Aktivitätszunahme der SSA, nicht aber der MSNA aus.

Zur Vermeidung einer übermäßigen Irritation des Nervs wurde die Suche nach dem MSNA-Signal auf 45 Minuten beschränkt. Bei Nicht-Auffinden des Nervs wurde das komplette Protokoll durchgeführt, allerdings ohne Aufzeichnung der MSNA.

\subsubsection{Datenerfassung}

Die Datenerfassung und Speicherung erfolgte durch das MIDAS-System (Modular Intensive Care Data Acquisition System, Institut für Biomedizinische Technik, Hochschule Mannheim, Germany). Das System besteht aus einem frei programmierbaren 16-Kanal-Verstärker und einem Analog-Digital-Wandler, der die Filterung, Verstärkung und Digitalisierung von Biosignalen ermöglicht. Der AnalogDigital-Wandler liefert auch bei gleichzeitiger Aufzeichnung mehrerer Signale mit hoher Frequenz und Amplitude eine hohe digitale Auflösung von 12 Bit. Außerdem können Parameter wie Amplitude, Abtastrate und Messbereich für jeden Kanal individuell definiert werden. Die digitalisierten Signale werden in einem FIFO(first in first out)-Zwischenspeicher abgelegt und zur weiteren Verarbeitung über einen USBAnschluss weitergeleitet. Die aufgezeichneten Kurven werden in einem binären Dateiformat gespeichert und im von Dr. Peter Hermann (Abteilung für AnästhesieTechnik, Universitätsmedizin Göttingen, Leitung: Prof. Dr. Michael Quintel) zur Verfügung gestellten Medical Device Integration System (MedIS) dargestellt. Außerdem werden Teile des Rohsignals als ASCII(American Standard Code for Information Interchange)-Tabelle gespeichert, die später in die Statistik-Software eingefügt werden kann. 


\subsection{Auswertung}

\subsubsection{MSNA}

Nach Bestätigung der korrekten Position der Elektrode wurde das über den A/DWandler auf das Notebook übertragene Signal aufgezeichnet. Das zeitliche Auftreten, die Form und die Frequenz eines sympathischen Nervensignals geben Aufschluss über den Ursprung des Signals:

- Signale der sympathischen Hautefferenzen imponieren als breitbasige, zum Teil mehrgipflige Spitzen.

- Signale der sympathischen muskulären Nervenaktivität sind charakteristischerweise schmal, beschreiben einen steilen Anstieg mit nur einer Spitze und treten kurz nach dem QRS-Komplex des simultan erfassten Elektrokardiogramms auf. Außerdem ist die MSNA-Frequenz durch die Atmung beeinflussbar. Eine langsame Atmung reduziert den Tonus der MSNA, wobei die Bursts in Gruppen während der Exspiration auftreten.

Die Interpretation der MSNA-Signale erfolgte verblindet durch zwei voneinander unabhängige Auswerter, sodass sowohl die Gruppenzugehörigkeit des Studienteilnehmers als auch die Intervention unbekannt waren. Es wurden vor der Auswertung Kriterien definiert, nach denen die Bursts als MSNA gewertet wurden.

- Die Amplitude der einzelnen Bursts muss mindestens doppelt so groß sein wie die Amplitude des in der Aufzeichnung des integrierten MultifaserSummenaktionspotentials bestehenden Grundrauschens.

- Charakteristisch für MSNA-Bursts ist weiter die Kopplung an die Diastole des Blutdrucks. Das Maximum des Bursts tritt dabei gleichzeitig mit dem Wiederanstieg des Blutdruckes zu Beginn der Systole auf.

- Parallel zum integrierten Multifaser-Summenaktionspotential wurde auch das nicht-integrierte Multifaser-Summenaktionspotential als Rohsignal aufgezeichnet. Das Auftreten eines Bursts im integrierten Signal wird begleitet von einer gleichzeitigen Zunahme der Amplitude des Rohsignals.

Ausgewertet wurden jeweils zweiminütige Intervalle, die repräsentativ für den jeweiligen Versuchsteil sind. Die Bursts wurden visuell anhand von Screenshots am Monitor ausgezählt und als Bursts pro Minute registriert. 


\subsubsection{Statistische Methoden}

Sämtliche statistische Berechnungen wurden mit dem Programm IBM SPSS Statistics 20 für Microsoft Windows durchgeführt.

Alle im Rahmen der Studie erhobenen Werte wurden als Mittelwerte (mean) mit Standardfehler des Mittelwertes (standard error of the mean, SEM) angegeben. Demographische Daten und Basis-Messwerte von Patienten und Probanden wurden mittels des zweiseitigen Student's t-test für gepaarte Stichproben miteinander verglichen.

Die gruppen- und zeitbezogenen Unterschiede der Messwerte für MSNA, BaroreflexSensitivität, Herzfrequenz, Blutdruck, Atmung und Blutgase zwischen den Gruppen und Interventionen wurden ebenfalls mittels eines zweiseitigen Student's t-test für gepaarte Stichproben hinsichtlich ihrer Signifikanz geprüft. Eine statistische Signifikanz wurde bei einem $\mathrm{p}<0,05$ erreicht. Die Speicherung der gesamten Daten erfolgte in digitaler Form.

\subsubsection{Power-Analyse}

Die Berechnung der für die Studie benötigten Teilnehmer ergab sich aus den Daten früherer Studien der Arbeitsgruppe (Raupach et al. 2008) (Andreas et al. 2004). Für jede der 3 Gruppen (COPD ohne NIV, COPD mit NIV und Kontrollgruppe) waren 10 Studienteilnehmer nötig, um einen auf dem 5\%-Niveau signifikanten Unterschied der MSNA um 10\% durch die Interventionen mit einer Power von $80 \%$ zu erkennen.

Da bei der Durchführung der Mikroneurographie, unabhängig von ausreichender Erfahrung und Praxis des Untersuchers, nur in 70\% der Untersuchungen ein adäquates Signal abgeleitet werden kann, mussten 15 Teilnehmer je Gruppe, also insgesamt 45 Patienten/Probanden in die Studie aufgenommen werden. 


\section{Ergebnisse}

\subsection{Teilnehmer-Charakteristika}

\subsubsection{Baseline-Charakteristika}

Im Rahmen der Studie wurden 15 gesunde Probanden und 30 Patienten mit COPD (jeweils 15 Patienten mit und ohne NIV) untersucht. Bezüglich des Alters, des Geschlechts und des Body Mass Index fanden sich keine signifikanten Unterschiede zwischen den Studiengruppen. Die Teilnehmer der drei Gruppen unterschieden sich allerdings signifikant bezüglich der in Ruhe gemessenen lungenfunktionellen Parameter (VC, $\mathrm{FEV}_{1} / \mathrm{VC}, \mathrm{FEV}_{1}, \mathrm{RV} / \mathrm{TLC}$ ) und des arteriellen $\mathrm{CO}_{2}$-Partialdruckes. Weiter bestand ein signifikanter Unterschied der Herzfrequenz, der arteriellen Pulswellengeschwindigkeit und der peripheren Sauerstoffsättigung von Kontrollprobanden und COPD-Patienten, nicht jedoch von COPD-Patienten ohne NIV und COPD-Patienten mit NIV (siehe Tabelle 4).

Wie erwartet zeigten sich in der Lungenfunktion signifikante Unterschiede zwischen den drei Gruppen. Die COPD-Patienten zeigten eine deutlich verminderte Vitalkapazität und ein signifikant erhöhtes Residualvolumen als Zeichen einer Lungenüberblähung. Auch die Einsekundenkapazität und der Tiffenau-Wert waren dem Grad der COPD entsprechend vermindert. Die COPD-Patienten mit NIV wiesen auch im Vergleich mit den COPD-Patienten ohne NIV signifikant schlechtere Lungenfunktionswerte auf.

Die Herzfrequenz der COPD-Patienten war im Vergleich zu den gesunden Kontrollprobanden signifikant erhöht. Zwischen den COPD-Patienten mit und ohne NIV fanden sich diesbezüglich keine statistisch signifikanten Unterschiede.

Die arterielle Pulswellengeschwindigkeit der COPD-Patienten war im Vergleich zu jener der gesunden Kontrollprobanden signifikant erhöht. Es konnte weiter eine höhere arterielle Pulswellengeschwindigkeit bei COPD-Patienten mit NIV gemessen werden, die sich aber nicht signifikant von der Pulswellengeschwindigkeit der COPDPatienten ohne NIV unterschied $(\mathrm{p}=0,061)$. 
Die periphere Sauerstoffsättigung der gesunden Kontrollprobanden war geringfügig höher als die der COPD-Patienten; der Unterschied erreichte knapp statistische Signifikanz $(p=0,044)$. Die $\mathrm{SpO}_{2}$-Werte beider Gruppen lagen aber im normoxischen Bereich. 


\begin{tabular}{|c|c|c|c|c|c|c|}
\hline & $\begin{array}{l}\text { Gesunde } \\
\text { Kontroll- } \\
\text { Probanden }\end{array}$ & $\begin{array}{l}\text { COPD- } \\
\text { Patienten } \\
(n=30)\end{array}$ & $\begin{array}{l}\text { p-Wert } \\
\text { (T-Test) }\end{array}$ & $\begin{array}{l}\text { Vergleich zv } \\
\text { Patienten m }\end{array}$ & $\begin{array}{l}\text { shen COPD- } \\
\text { nd ohne NIV }\end{array}$ & $\begin{array}{l}\text { p-Wert } \\
\text { (T-Test) }\end{array}$ \\
\hline \multirow{2}{*}{$\begin{array}{l}\text { Geschlecht (männlich / } \\
\text { weiblich) }\end{array}$} & \multirow{2}{*}{$9 / 6$} & \multirow{2}{*}{$20 / 10$} & \multirow{2}{*}{0,66} & ohne NIV & $9 / 6$ & \multirow{2}{*}{0,439} \\
\hline & & & & mit NIV & $11 / 4$ & \\
\hline \multirow{2}{*}{ Alter [Jahre] } & \multirow{2}{*}{$65,9 \pm 1,8$} & \multirow{2}{*}{$67,4 \pm 1,3$} & \multirow{2}{*}{0,524} & ohne NIV & $66,9 \pm 2,2$ & \multirow{2}{*}{0,698} \\
\hline & & & & mit NIV & $67,9 \pm 1,6$ & \\
\hline \multirow{2}{*}{ Größe [m] } & \multirow{2}{*}{$1,70 \pm 0,02$} & \multirow{2}{*}{$1,70 \pm 0,02$} & \multirow{2}{*}{0,937} & ohne NIV & $1,71 \pm 0,03$ & \multirow{2}{*}{0,331} \\
\hline & & & & mit NIV & $1,68 \pm 0,02$ & \\
\hline \multirow{2}{*}{ Gewicht [kg] } & \multirow{2}{*}{$74,5 \pm 3,0$} & \multirow{2}{*}{$80,9 \pm 3,0$} & \multirow{2}{*}{0,186} & ohne NIV & $82,1 \pm 3,7$ & \multirow{2}{*}{0,713} \\
\hline & & & & mit NIV & $79,8 \pm 4,8$ & \\
\hline \multirow{2}{*}{$\mathrm{BMI}\left[\mathrm{kg} / \mathrm{m}^{2}\right]$} & \multirow{2}{*}{$25,8 \pm 0,9$} & \multirow{2}{*}{$28,1 \pm 0,9$} & \multirow{2}{*}{0,13} & ohne NIV & $28,1 \pm 1,4$ & \multirow{2}{*}{0,95} \\
\hline & & & & mit NIV & $28,0 \pm 1,3$ & \\
\hline \multirow{2}{*}{$\begin{array}{l}\text { arterielle Pulswellen- } \\
\text { Geschwindigkeit [m/s] }\end{array}$} & \multirow{2}{*}{$6,2 \pm 0,4$} & \multirow{2}{*}{$8,9 \pm 0,5$} & 0002 & ohne NIV & $8,0 \pm 0,6$ & 0061 \\
\hline & & & 0,002 & mit NIV & $10,0 \pm 0,8$ & 0,061 \\
\hline Yc ro\% Colll & $000+25$ & $500+26$ & 0001 & ohne NIV & $66,7 \pm 3,2$ & 0001 \\
\hline $\operatorname{ve}[\% \mathrm{l} . \mathrm{SOII}]$ & Yo,0 $\pm<, 0$ & $30,9 \pm<, 0$ & $<0,001$ & mit NIV & $51,1 \pm 2,8$ & 0,001 \\
\hline EEV $/(C$ [о/] & $770+17$ & $160+20$ & $=0001$ & ohne NIV & $54,1 \pm 3,4$ & 001 \\
\hline$r E V_{1} / V C[\%]$ & $7 /, 2 \pm 1,1$ & $46,9 \pm 2,9$ & $<0,001$ & mit NIV & $39,7 \pm 3,9$ & 0,01 \\
\hline & & & & ohne NIV & $47,5 \pm 2,8$ & \\
\hline $\mathrm{FEV}_{1}[\%$ v. Soll $]$ & $102,0 \pm 3,2$ & $37,6 \pm 2,6$ & $<0,001$ & mit NIV & $27,7 \pm 2,5$ & $<0,001$ \\
\hline & & & & ohne NIV & $149,1 \pm 4,3$ & \\
\hline RV/ILC [\% V. Soll] & $100,9 \pm 4,0$ & $162,5 \pm 4,5$ & $<0,001$ & mit NIV & $175,9 \pm 6,2$ & 0.001 \\
\hline $\mathrm{m} \mathrm{CO}[\mathrm{mmHal}$ & $364+06$ & $130+08$ & $=0.001$ & ohne NIV & $40,1 \pm 0,7$ & $=0001$ \\
\hline$\rho_{2}\left[\mathrm{~m} \mathrm{U}_{2}\right.$ & $36,4 \pm 0,6$ & $43,0 \pm 0,8$ & $<\mathbf{U , U O I}$ & mit NIV & $45,9 \pm 1,1$ & $<0,001$ \\
\hline & & & & ohne NIV & $74,9 \pm 2,7$ & \\
\hline $\mathrm{pO}_{2}[\mathrm{~mm} \mathrm{mg}]$ & $78,3 \pm 2,6$ & $r 2,1 \pm 1,9$ & 0,061 & mit NIV & $69,3 \pm 2,4$ & $0,8 / 3$ \\
\hline & & & & ohne NIV & $69,2 \pm 2,4$ & \\
\hline Herztrequenz [min $\left.{ }^{-1}\right]$ & $60,3 \pm 2,0$ & $71,7 \pm 1,9$ & $<0,001$ & mit NIV & $75,0 \pm 2,9$ & 0,138 \\
\hline & & & & ohne NIV & $95,5 \pm 0,7$ & \\
\hline $\mathrm{SpO}_{2}[\%]$ & $96,6 \pm 0,5$ & $95,3 \pm 0,4$ & 0,044 & mit NIV & $95,0 \pm 0,5$ & 0,551 \\
\hline systolischer Blutdruck & $1428+88$ & $1383+42$ & 0604 & ohne NIV & $142,2 \pm 61$ & 0304 \\
\hline (Portapres) [mmHg] & $14<, 0 \pm 0,0$ & $100,0 \pm 4,2$ & 0,004 & mit NIV & $133,4 \pm 5,4$ & 0,004 \\
\hline diastolischer Blutdruck & $721+10$ & 717 , $>$ & 0805 & ohne NIV & $75,5 \pm 3,7$ & 0120 \\
\hline (Portapres) [mmHg] & $r 2,4 \pm 4,9$ & $7, I \pm 2, I$ & 0,895 & mit NIV & $66,9 \pm 3,9$ & 0,120 \\
\hline Atomfrogun $7 \mathrm{~min}^{-1}$ & $148+09$ & $150+10$ & 70 & ohne NIV & $14,9 \pm 1,3$ & 05 \\
\hline AlemiliequenL [IIII) ] & $14,0 \pm 0, \Xi$ & (2, & 0,019 & mit NIV & $15,5 \pm 1,0$ & 0,000 \\
\hline
\end{tabular}

Tabelle 4: Baseline-Charakteristika der 45 Studienteilnehmer 


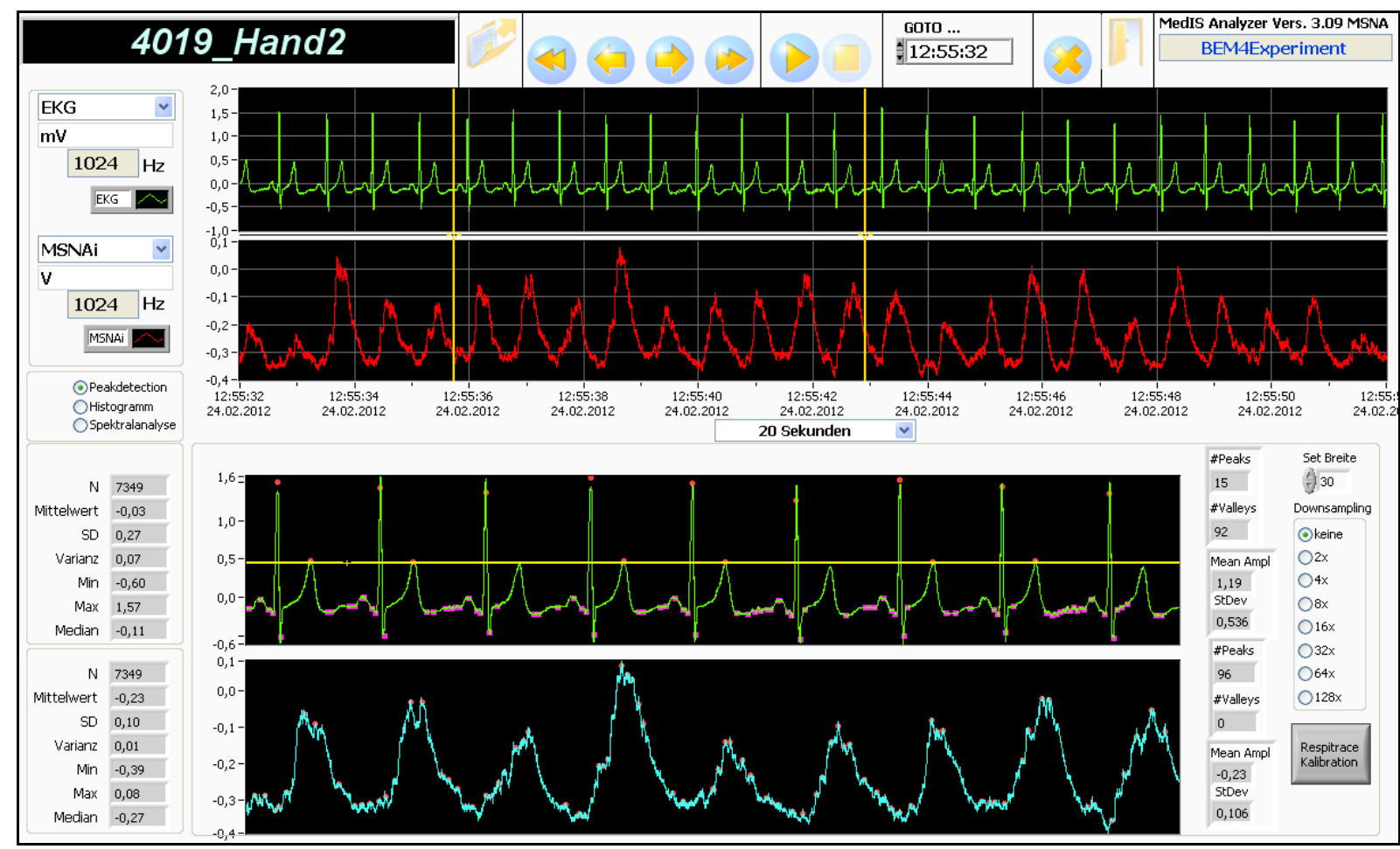

Abbildung 2: 20-sekündiger Ausschnitt aus der kontinuierlichen simultanen Aufzeichnung des Elektrokardiogramms (grün) und des integrierten MSNA-Signals (rot) bei einem COPD-Patienten; Im unteren Teil vergrößertes 7-sekündiges Intervall (Quelle: Medical Device Integration System (MeDIS), Abteilung für AnästhesieTechnik der Universitätsmedizin Göttingen, Leiter: Prof. Dr. Michael Quintel)

\subsubsection{MSNA in Ruhe}

Ein MSNA-Signal konnte bei 13 COPD-Patienten ohne NIV, bei 5 COPD-Patienten mit NIV und bei 9 gesunden Kontrollprobanden aufgezeichnet werden.

Die in Ruhe gemessene Baseline-Aktivität der MSNA war bei den COPD-Patienten (36,9 $\pm 2,1$ Bursts/min) durchgängig signifikant höher als in der Kontrollgruppe $(27,6$ $\pm 2,4$ Bursts/min) (siehe Tabelle 5). Innerhalb der Gruppe der COPD-Patienten zeigten die Patienten mit NIV (41,8 \pm 1,8 Bursts/min) auch gegenüber den COPDPatienten ohne NIV $(33,7 \pm 2,6$ Bursts/min) eine signifikant gesteigerte MSNA. 


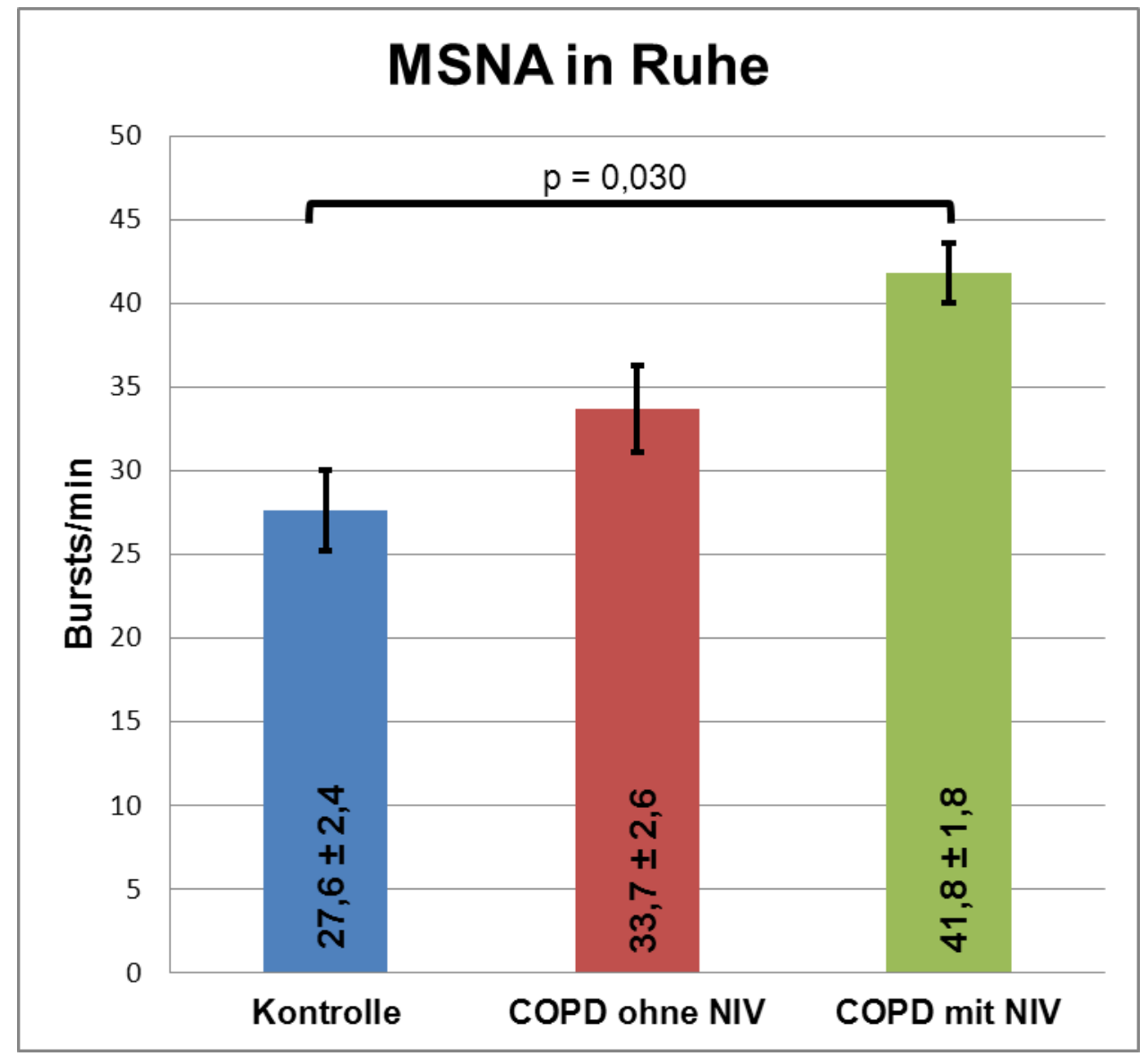

Abbildung 3: MSNA in Ruhe im Vergleich der drei Studiengruppen. Die Werte in den Säulen geben den Mittelwert mit Standardfehler an. Der angegebene $p$-Wert bezieht sich auf den zweiseitigen Student`s t-Test für gepaarte Stichproben.

\begin{tabular}{|c|c|c|c|c|c|c|}
\hline & COPD & Kontrolle & $p$-Wert & $\begin{array}{r}\text { Vergleic } \\
\text { COPD-P } \\
\text { und o } \\
\end{array}$ & $\begin{array}{l}\text { zwischen } \\
\text { ienten mit } \\
\text { ne NIV }\end{array}$ & $p$-Wert \\
\hline \multirow{2}{*}{ Ruhe } & \multirow{2}{*}{$36,9 \pm 2,1$} & \multirow{2}{*}{$27,6 \pm 2,4$} & \multirow{2}{*}{0,05} & ohne NIV & $33,7 \pm 2,6$ & \multirow{2}{*}{$<0,05$} \\
\hline & & & & mit NIV & $41,8 \pm 1,8$ & \\
\hline \multirow{2}{*}{$\begin{array}{l}\text { Dynamischer } \\
\text { Handgrip }\end{array}$} & \multirow{2}{*}{$40,9 \pm 3,7$} & \multirow{2}{*}{$27,6 \pm 2,5$} & \multirow{2}{*}{$<0,05$} & ohne NIV & $34,5 \pm 1,8$ & \multirow{2}{*}{0,06} \\
\hline & & & & mit NIV & $51,6 \pm 5,0$ & \\
\hline \multirow{2}{*}{$\begin{array}{l}\text { Statischer } \\
\text { Handgrip }\end{array}$} & \multirow{2}{*}{$45,2 \pm 4,0$} & \multirow{2}{*}{$32,6 \pm 1,5$} & \multirow{2}{*}{$<0,05$} & ohne NIV & $37,1 \pm 2,2$ & \multirow{2}{*}{$<0,05$} \\
\hline & & & & mit NIV & $55,5 \pm 4,8$ & \\
\hline
\end{tabular}

Tabelle 5: MSNA bei COPD und Kontrolle

\subsubsection{Baroreflexsensitivität}

Die ermittelten Werte der Baroreflexsensitivität zeigten bei den COPD-Patienten (4,6 $\pm 0,5 \mathrm{~ms} / \mathrm{mmHg})$ bereits in Ruhe eine im Vergleich zur Kontrollgruppe $(7,6 \pm 1,4$ 
$\mathrm{ms} / \mathrm{mmHg})$ signifikant verminderte Baroreflexsensitivität $(p=0,004)$. Zwischen COPDPatienten mit und ohne NIV ergab sich hier jedoch kein signifikanter Unterschied.

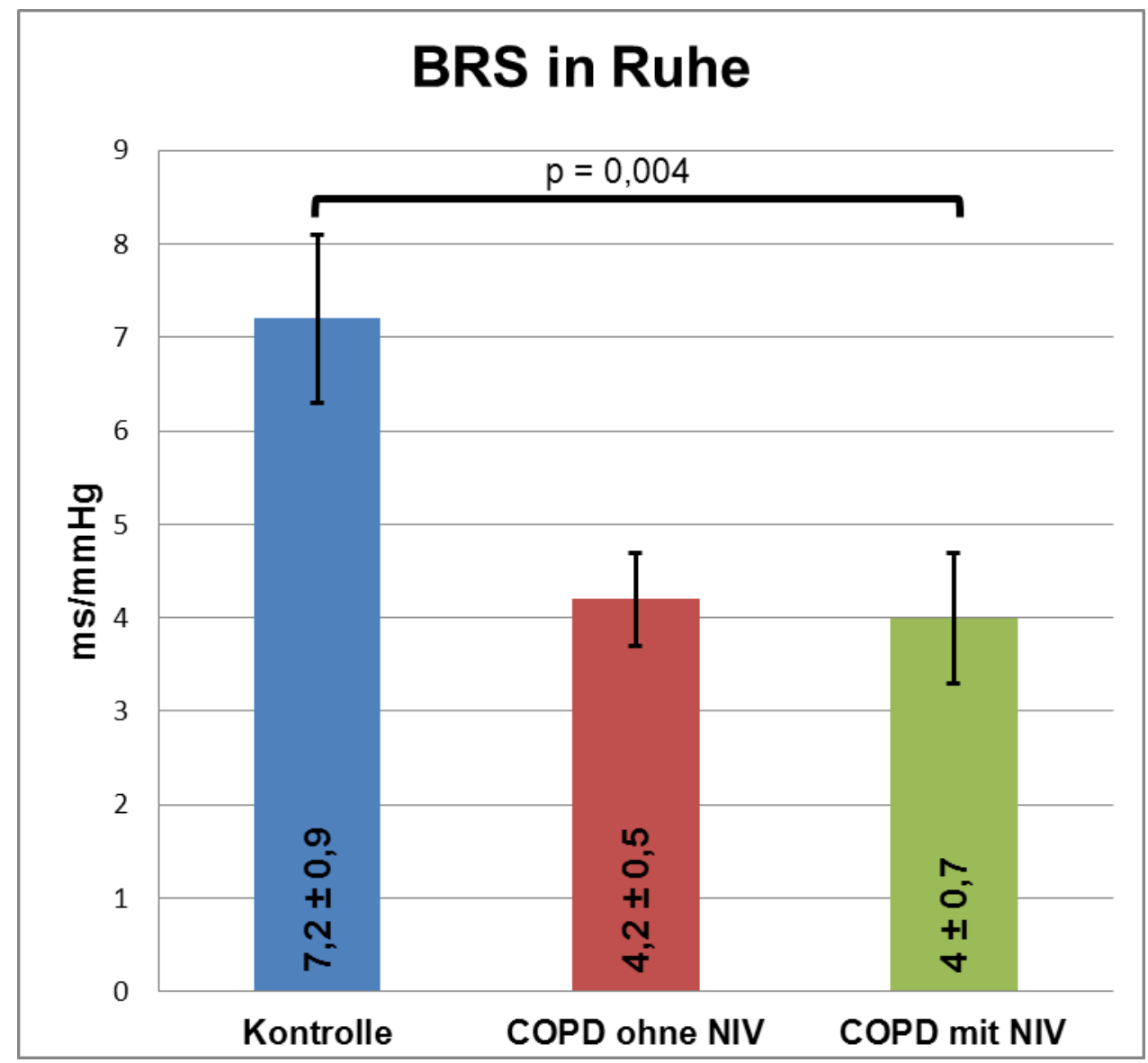

Abbildung 4: Baroreflexsensitivität in Ruhe im Vergleich der drei Studiengruppen. Die Werte in den Säulen geben den Mittelwert mit Standardfehler an. Der angegebene $\mathrm{p}$-Wert bezieht sich auf den zweiseitigen Student`s t-Test für gepaarte Stichproben.

\subsubsection{Spiroergometrie}

Die in der Spiroergometrie erhobenen Daten zeigten signifikante Unterschiede zwischen gesunden Kontrollprobanden und COPD-Patienten. Besonders ausgeprägt waren die Differenzen zwischen COPD-Patienten mit nicht-invasiver Beatmung und gesunden Kontrollprobanden (siehe Tabelle 6). 
Das ventilatorische Äquivalent für $\mathrm{CO}_{2}\left(\mathrm{VE} / \mathrm{VCO}_{2}\right)$ unter maximaler Belastung war bei COPD-Patienten mit 32,0 \pm 0,9 im Vergleich zur Kontrollgruppe mit 28,9 $\pm 0,9$ signifikant erhöht. Die Werte der COPD-Patienten mit NIV wichen nicht signifikant von denen der COPD-Patienten ohne NIV ab.

Auch die maximale Leistungsfähigkeit der COPD-Patienten (36,4 \pm 6,2 Watt) war im Vergleich zur Kontrollgruppe $(110,3 \pm 8,7$ Watt) deutlich herabgesetzt. COPDPatienten mit NIV (12,5 \pm 1,8 Watt) zeigten im Vergleich zu COPD-Patienten ohne NIV $(49,2 \pm 7,6$ Watt) nochmals signifikant niedrigere Werte.

Deutliche Einschränkungen zeigten sich bei den COPD-Patienten auch in der maximalen $\mathrm{O}_{2}$-Aufnahme. Mit 11,9 \pm 1,0 $\mathrm{l} /$ min wichen die COPD-Patienten deutlich von der Kontrollgruppe $(23,2 \pm 1,5 \mathrm{l} / \mathrm{min})$ ab. Die COPD-Patienten mit NIV erreichten erneut signifikant niedrigere Werte als die COPD-Patienten ohne NIV.

\begin{tabular}{|c|c|c|c|c|c|c|}
\hline & COPD & Kontrolle & $\begin{array}{c}\text { p- } \\
\text { Wert }\end{array}$ & $\begin{array}{r}\text { Vergleic } \\
\text { COPD-P } \\
\text { und c }\end{array}$ & $\begin{array}{l}\text { zwischen } \\
\text { ienten mit } \\
\text { ne NIV }\end{array}$ & $\begin{array}{c}\mathrm{p}- \\
\text { Wert }\end{array}$ \\
\hline \multirow{2}{*}{$\begin{array}{l}\mathrm{VE} / \mathrm{VCO}_{2} \text { (max. } \\
\text { Belastung) }\end{array}$} & \multirow{2}{*}{$32,0 \pm 0,9$} & \multirow{2}{*}{$28,9 \pm 0,9$} & \multirow{2}{*}{$<0,05$} & ohne NIV & $31,9 \pm 1,3$ & \multirow{2}{*}{0,861} \\
\hline & & & & mit NIV & $32,3 \pm 1,2$ & \\
\hline \multirow{2}{*}{$\begin{array}{l}\max . \\
\text { Leistungsfähig- } \\
\text { keit [W] }\end{array}$} & \multirow[b]{2}{*}{$36,4 \pm 6,2$} & \multirow{2}{*}{$110,3 \pm 8,7$} & \multirow[b]{2}{*}{$<0,05$} & ohne NIV & $49,2 \pm 7,6$ & \multirow[b]{2}{*}{$<0,05$} \\
\hline & & & & mit NIV & $12,5 \pm 1,8$ & \\
\hline \multirow{2}{*}{$\begin{array}{l}\max . \\
\mathrm{O}_{2} \text {-Aufnahme } \\
{[\mathrm{l} / \mathrm{min}]}\end{array}$} & \multirow[b]{2}{*}{$11,9 \pm 1,0$} & \multirow{2}{*}{$23,2 \pm 1,5$} & \multirow{2}{*}{$<0,05$} & ohne NIV & $13,5 \pm 1,3$ & \multirow{2}{*}{$<0,05$} \\
\hline & & & & mit NIV & $8,9 \pm 0,6$ & \\
\hline
\end{tabular}

Tabelle 6: Spiroergometrie bei COPD und Kontrolle

Im Folgenden sind die Ergebnisse anhand der in Abschnitt 1.6 formulierten Hypothesen dargestellt. 


\subsection{Hypothese 1:}

Die MSNA in Ruhe korreliert bei Gesunden und COPD-Patienten mit dem ventilatorischen Äquivalent für $\mathrm{CO}_{2}\left(\mathrm{VE} / \mathrm{VCO}_{2}\right)$, der maximalen $\mathrm{O}_{2}$-Aufnahme und der körperlichen Belastbarkeit in der Spiroergometrie auf dem Standfahrrad.

Die Ergebnisse der Korrelationsanalysen bezüglich der Ruhe-MSNA und verschiedener spiroergometrischer Parameter sind in Tabelle 7 dargestellt. Die COPD-Patienten wiesen eine im Vergleich zur Kontrollgruppe signifikant gesteigerte MSNA in Ruhe auf. Außerdem war die maximale $\mathrm{O}_{2}$-Aufnahme unter höchster Belastung im Vergleich zur Kontrollgruppe signifikant vermindert. Das $\mathrm{VE} / \mathrm{VCO}_{2}$ unter maximaler Belastung in der Spiroergometrie war bei den COPD-Patienten signifikant höher als in der Kontrollgruppe. Die Korrelation der MSNA in Ruhe mit der maximalen $\mathrm{O}_{2}$-Aufnahme sowie der $\mathrm{VE} / \mathrm{VCO}_{2}$ innerhalb der beiden Studiengruppen erreichte zwar keine statistische Signifikanz, jedoch fällt auf, dass $\mathrm{VE} / \mathrm{VCO}_{2}$ und MSNA in Ruhe bei COPD-Patienten negativ korreliert ist, während die Korrelation in der Kontrollgruppe positiv ist.

Auch für die maximale Leistungsfähigkeit konnte weder bei den COPD-Patienten noch bei den gesunden Kontrollprobanden eine signifikante Korrelation mit der RuheMSNA gemessen werden. Allerdings fand sich in der Gesamtgruppe aller Studienteilnehmer (COPD-Patienten und gesunde Kontrollprobanden) eine signifikante negative Korrelation zwischen der maximalen Leistungsfähigkeit und der Ruhe-MSNA.

Im gesamten Studienkollektiv bestand also ein signifikanter negativer Zusammenhang zwischen der Ruheaktivität des sympathischen Nervensystems und der maximalen körperlichen Leistungsfähigkeit in der Spiroergometrie. 


\begin{tabular}{|l|c|c|c|c|c|c|}
\hline \multirow{2}{*}{$\begin{array}{l}\text { Korrelation mit } \\
\text { MSNA in Ruhe } \\
\text { [Bursts/min] }\end{array}$} & \multicolumn{2}{|c|}{ COPD } & \multicolumn{2}{c|}{ Kontrolle } & \multicolumn{2}{c|}{ Gesamtgruppe } \\
\cline { 2 - 7 } & $\begin{array}{c}\text { Pearson`s } \\
r\end{array}$ & $p$-Wert & $\begin{array}{c}\text { Pearson`s } \\
r\end{array}$ & $p$-Wert & $\begin{array}{c}\text { Pearson`s } \\
r\end{array}$ & $p$-Wert \\
\hline $\begin{array}{l}\text { VE/VCO } \\
\text { Belastung) (max. }\end{array}$ & 0,287 & 0,491 & $-0,353$ & 0,561 & 0,002 & 0,994 \\
\hline $\begin{array}{l}\text { max. } \\
\text { Leistungsfähigkeit } \\
\text { [W] }\end{array}$ & $-0,173$ & 0,656 & $-0,308$ & 0,614 & $-\mathbf{0 , 5 5 2}$ & $\mathbf{0 , 0 4 1}$ \\
\hline $\begin{array}{l}\text { max. } \\
\begin{array}{l}\mathrm{O}_{2} \text {-Aufnahme } \\
\text { [l/min] }\end{array}\end{array}$ & $-0,102$ & 0,795 & $-0,468$ & 0,427 & $-0,484$ & 0,080 \\
\hline
\end{tabular}

Tabelle 7: Korrelation von MSNA und Spiroergometrie

\subsection{Hypothese 2:}

Die Steigerung der MSNA und die Abnahme der Baroreflexsensitivität während einer Handkontraktionsübung als Äquivalent leichter körperlicher Belastung sind bei COPD-Patienten größer als bei gesunden Probanden.

Um Veränderungen der MSNA und der Baroreflexsensitivität bei gesunden Kontrollprobanden und COPD-Patienten miteinander vergleichen zu können, wurden jeweils zweiminütige Intervalle in Ruhe und unter dynamischer beziehungsweise statischer Handgrip-Belastung aufgezeichnet und dann jeweils die Veränderung zwischen den Ruhe- und den Belastungsphasen mittels gepaarter T-Tests untersucht.

Unter dynamischer Handgrip-Belastung wiesen die COPD-Patienten eine im Vergleich zu den gesunden Kontrollprobanden signifikant höhere MSNA auf. Sowohl bei den gesunden Kontrollprobanden als auch bei den COPD-Patienten ließ sich unter dynamischer Belastung ein Anstieg der MSNA gegenüber den Werten in Ruhe feststellen, der allerdings keine Signifikanz erreichte. 


\begin{tabular}{|l|c|c|c|}
\hline $\begin{array}{c}\text { MSNA } \\
\text { [Bursts/min] }\end{array}$ & Erste Ruhephase & $\begin{array}{c}\text { Dynamischer } \\
\text { Handgrip }\end{array}$ & $\begin{array}{c}\text { p-Wert (gepaarter } \\
\text { T-Test) }\end{array}$ \\
\hline COPD & $37,0 \pm 2,5$ & $40,9 \pm 3,7$ & 0,189 \\
\hline Kontrolle & $26,1 \pm 2,4$ & $27,6 \pm 2,5$ & 0,447 \\
\hline
\end{tabular}

Tabelle 8: MSNA [Bursts/min] in Ruhe und unter dynamischer Belastung

Auch unter statischer Handgrip-Belastung ließ sich bei den COPD-Patienten eine im Vergleich zur Kontrollgruppe signifikant höhere sympathische Nervenaktivität messen. Weiter zeigten die COPD-Patienten mit NIV eine signifikant höhere MSNA (55,5 \pm 4,8 Bursts/min) als COPD-Patienten ohne NIV (37,1 $\pm 2,2$ Bursts/min) $(\mathrm{p}<0,05)$.

Bei den gesunden Kontrollprobanden fand unter statischer Belastung keine signifikante Veränderung der MSNA im Vergleich zur Messung in Ruhe statt, während die COPD-Patienten mit einem signifikanten Anstieg $(p=0,022)$ der MSNA auf die statische Handgrip-Belastung reagierten.

\begin{tabular}{|l|c|c|c|}
\hline $\begin{array}{c}\text { MSNA } \\
\text { [Bursts/min] }\end{array}$ & Zweite Ruhephase & $\begin{array}{c}\text { Statischer } \\
\text { Handgrip }\end{array}$ & $\begin{array}{c}\text { p-Wert (gepaarter } \\
\text { T-Test) }\end{array}$ \\
\hline COPD & $37,4 \pm 2,3$ & $45,2 \pm 4,0$ & $\mathbf{0 , 0 2 2}$ \\
\hline Kontrolle & $29,4 \pm 2,0$ & $32,6 \pm 1,5$ & 0,351 \\
\hline
\end{tabular}

Tabelle 9: MSNA [Bursts/min] in Ruhe und unter statischer Belastung

Der Veränderung der MSNA bei den COPD-Patienten zwischen Ruhe und statischer Handgrip-Belastung war ausgeprägter als die Veränderung der MSNA zwischen Ruhe und dynamischer Handgrip-Belastung. Weiter lag die MSNA der COPDPatienten zu jedem Zeitpunkt der Messung über der Aktivität der Kontrollgruppe.

Die Baroreflexsensitivität der COPD-Patienten war zu jedem Zeitpunkt der Aufzeichnung geringer als die der gesunden Kontrollprobanden, die Differenz 
erreichte aber nur in der ersten Ruhephase $(p=0,005)$ und unter statischer HandgripBelastung $(p=0,020)$ statistische Signifikanz.

Unter dynamischer Belastung konnte eine Abnahme der Baroreflexsensitivität gegenüber den Ruhewerten sowohl bei den COPD-Patienten als auch bei den Kontrollprobanden gemessen werden. Die Veränderungen erreichten aber keine statistische Signifikanz (siehe Tabelle 10).

Auch unter statischer Handgrip-Belastung kam es in der Kontrollgruppe zu einer Abnahme der Baroreflexsensitivität, während bei den COPD-Patienten ein Anstieg der Baroreflexsensitivität gemessen wurde. Die Veränderungen erreichten aber ebenfalls keine statistische Signifikanz (siehe Tabelle 11).

\begin{tabular}{|l|c|c|c|}
\hline $\begin{array}{c}\text { Baroreflexsensitivität } \\
{[\mathbf{m s} / \mathbf{m m H g}]}\end{array}$ & Erste Ruhephase & $\begin{array}{c}\text { Dynamischer } \\
\text { Handgrip }\end{array}$ & $\begin{array}{c}\text { p-Wert (gepaarter } \\
\text { T-Test) }\end{array}$ \\
\hline COPD & $4,6 \pm 0,5$ & $4,3 \pm 0,9$ & 0,733 \\
\hline Kontrolle & $7,6 \pm 1,4$ & $7,5 \pm 2,1$ & 0,966 \\
\hline
\end{tabular}

Tabelle 10: Baroreflexsensitivität $[\mathrm{ms} / \mathrm{mmHg}]$ in Ruhe und unter dynamischer Belastung

\begin{tabular}{|l|c|c|c|}
\hline $\begin{array}{c}\text { Baroreflexsensitivität } \\
\text { [ms/mmHg] }\end{array}$ & Zweite Ruhephase & $\begin{array}{c}\text { Statischer } \\
\text { Handgrip }\end{array}$ & $\begin{array}{c}\text { p-Wert (gepaarter } \\
\text { T-Test) }\end{array}$ \\
\hline COPD & $4,3 \pm 0,5$ & $4,8 \pm 0,9$ & 0,503 \\
\hline Kontrolle & $7,2 \pm 0,9$ & $5,6 \pm 1,0$ & 0,122 \\
\hline
\end{tabular}

Tabelle 11: Baroreflexsensitivität [ms $/ \mathrm{mmHg}]$ in Ruhe und unter statischer Belastung

\subsection{Hypothese 3:}

Die Steigerung der MSNA und die Abnahme der Baroreflexsensitivität während der Handkontraktionsübungen korrelieren mit den in der Spiroergometrie erhobenen Daten. 
Um einen möglichen Zusammenhang zwischen einem Anstieg der MSNA-Frequenz unter dynamischer beziehungsweise statischer Handgrip-Belastung und Veränderungen in der Spiroergometrie zu untersuchen, wurden Differenzen in der MSNA-Frequenz zwischen Ruhe und leichter körperlicher Belastung mit den in der Spiroergometrie gewonnenen Daten korreliert. Im Fokus lagen hier insbesondere die maximale Leistungsfähigkeit und die maximale $\mathrm{O}_{2}$-Aufnahme. Neben der gesamten Studiengruppe aus allen COPD-Patienten und Kontrollprobanden mit ableitbarem MSNA-Signal $(n=27)$ wurden die beiden Untergruppen (COPD: $n=18$; Kontrolle: $n$ =9) auch getrennt voneinander untersucht.

Bei den COPD-Patienten zeigte sich eine signifikante negative Korrelation der maximalen Leistungsfähigkeit mit dem Anstieg der MSNA-Frequenz unter dynamischer Handgrip-Belastung. Auch in der Gesamtgruppe bestand eine negative Korrelation, während sich in der Subgruppe der gesunden Probanden kein statistisch signifikanter Zusammenhang darstellen ließ (siehe Tabelle 12).

Der Anstieg der MSNA unter statischer Handgrip-Belastung korrelierte in keiner der Teilgruppen oder der Gesamtgruppe signifikant mit der maximalen Leistungsfähigkeit.

\begin{tabular}{|l|c|c|c|c|c|c|}
\hline \multirow{2}{*}{$\begin{array}{l}\text { Korrelation mit } \\
\text { max. Leistung } \\
\text { [W] }\end{array}$} & \multicolumn{2}{|c|}{ COPD } & \multicolumn{2}{c|}{ Kontrolle } & \multicolumn{2}{c|}{ Gesamtgruppe } \\
\cline { 2 - 7 } & $\begin{array}{c}\text { Pearson`s } \\
\mathrm{r}\end{array}$ & $\mathrm{p}$-Wert & $\begin{array}{c}\text { Pearson`s } \\
\mathrm{r}\end{array}$ & $\begin{array}{c}\mathrm{p} \text { - } \\
\text { Wert }\end{array}$ & $\begin{array}{c}\text { Pearson`s } \\
\mathrm{r}\end{array}$ & $\mathrm{p}$-Wert \\
\hline $\begin{array}{l}\text { Anstieg der MSNA } \\
\text { (gegenüber Ruhe) } \\
\text { unter dynamischer }\end{array}$ & $-\mathbf{- 0 , 8 5 7}$ & $\mathbf{0 , 0 1 4}$ & $-0,431$ & 0,569 & $\mathbf{- 0 , 6 2 9}$ & $\mathbf{0 , 0 3 8}$ \\
$\begin{array}{l}\text { Handgrip- } \\
\text { Belastung }\end{array}$ & 0,15 & $-0,319$ & 0,681 & $-0,500$ & 0,098 \\
\hline $\begin{array}{l}\text { Anstieg der MSNA } \\
\text { (gegenüber Ruhe) } \\
\text { unter statischer } \\
\text { Handgrip- } \\
\text { Belastung }\end{array}$ & $-0,559$ & & & & & \\
\hline
\end{tabular}

Tabelle 12: Korrelation von max. Leistungsfähigkeit mit Anstieg der MSNA 
Obwohl für die maximale $\mathrm{O}_{2}$-Aufnahme weder bei den COPD-Patienten noch bei der gesunden Kontrollgruppe eine statistisch signifikante Korrelation mit dem Anstieg der MSNA unter dynamischer oder statischer Handgrip-Belastung besteht (Tabelle 13), fällt auf, dass bei COPD-Patienten eine negative, für die gesunde Kontrollgruppe eine positive Korrelation vorliegt.

\begin{tabular}{|c|c|c|c|c|c|c|}
\hline \multirow{2}{*}{$\begin{array}{l}\text { Korrelation mit } \\
\text { max. } \\
\text { Aufnahme } \\
{\left[\mathrm{OO}_{2} \text { max/kg KG] }\right.}\end{array}$} & \multicolumn{2}{|c|}{ COPD } & \multicolumn{2}{|c|}{ Kontrolle } & \multicolumn{2}{|c|}{ Gesamtgruppe } \\
\hline & $\begin{array}{c}\text { Pearson's } \\
r\end{array}$ & $\mathrm{p}$-Wert & $\begin{array}{c}\text { Pearson`s } \\
r\end{array}$ & $\begin{array}{c}p- \\
\text { Wert }\end{array}$ & $\begin{array}{c}\text { Pearson`s } \\
r\end{array}$ & $p-$ Wert \\
\hline $\begin{array}{l}\text { Anstieg der MSNA } \\
\text { (gegenüber Ruhe) } \\
\text { unter dynamischer } \\
\text { Handgrip- } \\
\text { Belastung }\end{array}$ & $-0,683$ & 0,091 & 0,428 & 0,572 & $-0,537$ & 0,089 \\
\hline $\begin{array}{l}\text { Anstieg der MSNA } \\
\text { (gegenüber Ruhe) } \\
\text { unter statischer } \\
\text { Handgrip- } \\
\text { Belastung }\end{array}$ & $-0,541$ & 0,166 & 0,542 & 0,458 & $-0,380$ & 0,223 \\
\hline
\end{tabular}

Tabelle 13: Korrelation von max. $\mathrm{O}_{2}$-Aufnahme mit Anstieg der MSNA

Zwar erreichten die Korrelationen von Differenzen der Baroreflexsensitivität unter Handgrip-Belastung mit der maximalen Leistungsfähigkeit oder der maximalen $\mathrm{O}_{2}-$ Aufnahme in der Spiroergometrie keine statistische Signifikanz, es ließ sich aber auch hier ein Trend feststellen. Während die Veränderungen der Baroreflexsensitivität unter Handgrip-Belastung bei der Kontrollgruppe positiv mit der maximalen Leistungsfähigkeit und $\mathrm{O}_{2}$-Aufnahme korrelierten, lagen bei den COPDPatienten ausschließlich negative Korrelationen vor (siehe Tabelle 14 und 15). 


\begin{tabular}{|l|c|c|c|c|c|c|}
\hline \multirow{2}{*}{$\begin{array}{l}\text { Korrelation mit } \\
\text { max. Leistung } \\
\text { [W] }\end{array}$} & \multicolumn{2}{|c|}{ COPD } & \multicolumn{2}{c|}{ Kontrolle } & \multicolumn{2}{c|}{ Gesamtgruppe } \\
\cline { 2 - 7 } & $\begin{array}{c}\text { Pearson`s } \\
r\end{array}$ & $p$-Wert & $\begin{array}{c}\text { Pearson`s } \\
r\end{array}$ & $\begin{array}{c}p- \\
\text { Wert }\end{array}$ & $\begin{array}{c}\text { Pearson`s } \\
r\end{array}$ & $p$-Wert \\
\hline $\begin{array}{l}\text { Differenz der BRS } \\
\text { (gegenüber Ruhe) } \\
\text { unter dynamischer } \\
\text { Belastung }\end{array}$ & $-0,171$ & 0,636 & 0,193 & 0,715 & 0,144 & 0,596 \\
\hline $\begin{array}{l}\text { Differenz der BRS } \\
\text { (gegenüber Ruhe) } \\
\text { unter statischer } \\
\text { Belastung }\end{array}$ & $-0,458$ & 0,13 & 0,003 & 0,993 & 0,059 & 0,793 \\
\hline
\end{tabular}

Tabelle 14:Korrelation von max. Leistungsfähigkeit mit Differenz der BRS

\begin{tabular}{|c|c|c|c|c|c|c|}
\hline \multirow{2}{*}{$\begin{array}{ll}\text { Korrelation mit } \\
\text { max. } \\
\text { Aufnahme } \\
{\left[\mathrm{VO}_{2} \text { max/kg KG] }\right.}\end{array}$} & \multicolumn{2}{|c|}{ COPD } & \multicolumn{2}{|c|}{ Kontrolle } & \multicolumn{2}{|c|}{ Gesamtgruppe } \\
\hline & $\underset{r}{\text { Pearson's }}$ & $\mathrm{p}$-Wert & $\begin{array}{c}\text { Pearson's } \\
r\end{array}$ & $\begin{array}{c}p- \\
\text { Wert }\end{array}$ & $\begin{array}{c}\text { Pearson's } \\
r\end{array}$ & $p$-Wert \\
\hline $\begin{array}{l}\text { Differenz der BRS } \\
\text { (gegenüber Ruhe) } \\
\text { unter dynamischer } \\
\text { Belastung }\end{array}$ & $-0,181$ & 0,617 & 0,096 & 0,856 & 0,098 & 0,717 \\
\hline $\begin{array}{l}\text { Differenz der BRS } \\
\text { (gegenüber Ruhe) } \\
\text { unter statischer } \\
\text { Belastung }\end{array}$ & $-0,375$ & 0,256 & 0,311 & 0,352 & 0,191 & 0,394 \\
\hline
\end{tabular}

Tabelle 15:Korrelation von max. $\mathrm{O}_{2}$-Aufnahme mit Differenz der BRS

Insgesamt ließ sich also ein - in einigen Analysen auch statistisch signifikanter inverser Zusammenhang zwischen gesteigerter sympathischer Reaktion auf dynamische Handgrip-Belastung und maximaler körperlicher Leistungsfähigkeit in der Spiroergometrie feststellen. 


\subsection{Hypothese 4:}

COPD-Patienten weisen nach der Benutzung nicht-invasiver Beatmung eine niedrigere MSNA und eine höhere Baroreflexsensitivität in Ruhe und unter leichter körperlicher Belastung auf als vor der Benutzung.

Ein möglicher Effekt der nicht-invasiven Beatmung auf die MSNA und die Baroreflexsensitivität wurde durch eine Gegenüberstellung der MSNA beziehungsweise der Baroreflexsensitivität der COPD-Patienten mit NIV in Ruhe und unter leichter körperlicher Handgrip-Belastung vor und nach der Nutzung der Beatmung untersucht. Die Frequenz der MSNA unter statischer und dynamischer Handgrip-Belastung nach Nutzung der nicht-invasiven Beatmung lag zwar unter der Frequenz vor der NIV-Benutzung, allerdings war der Effekt nicht statistisch signifikant.

\begin{tabular}{|l|c|c|c|}
\hline MSNA [Bursts/min] & $\begin{array}{c}\text { MSNA [Bursts/min] } \\
\text { vor NIV }\end{array}$ & $\begin{array}{c}\text { MSNA [Bursts/min] } \\
\text { nach NIV }\end{array}$ & p-Wert \\
\hline Ruhe & $41,8 \pm 1,8$ & $45,1 \pm 5,7$ & 0,556 \\
\hline $\begin{array}{l}\text { statische Handgrip- } \\
\text { Belastung }\end{array}$ & $55,5 \pm 5,8$ & $52,9 \pm 7,9$ & 0,304 \\
\hline $\begin{array}{l}\text { dynamische } \\
\text { Handgrip-Belastung }\end{array}$ & $51,6 \pm 5,0$ & $46,4 \pm 2,7$ & 0,578 \\
\hline
\end{tabular}

Tabelle 16: MSNA [Bursts/min] vor und nach Anwendung nicht-invasiver Beatmung

Auch auf die Baroreflexsensitivität konnte kein signifikanter Effekt der nicht-invasiven Beatmung dargestellt werden. Hier ließ sich keine einheitliche Tendenz vor und nach Beatmung nachweisen. 


\begin{tabular}{|l|c|c|c|}
\hline $\begin{array}{l}\text { Baroreflexsensitivität } \\
\text { [ms/mmHg] }\end{array}$ & $\begin{array}{c}\text { BRS [ms } / \mathrm{mmHg}] \\
\text { vor NIV }\end{array}$ & $\begin{array}{c}\text { BRS [ms } / \mathrm{mmHg}] \\
\text { nach NIV }\end{array}$ & p-Wert \\
\hline Ruhe & $4,2 \pm 0,8$ & $6,2 \pm 1,5$ & 0,265 \\
\hline $\begin{array}{l}\text { statische Handgrip- } \\
\text { Belastung }\end{array}$ & $3,3 \pm 0,8$ & $3,6 \pm 1,0$ & 0,635 \\
\hline $\begin{array}{l}\text { dynamische Handgrip- } \\
\text { Belastung }\end{array}$ & $5,0 \pm 1,5$ & $3,0 \pm 0,5$ & 0,508 \\
\hline
\end{tabular}

Tabelle 17: Baroreflexsensitivität $[\mathrm{ms} / \mathrm{mmHg}$ ] vor und nach Anwendung nichtinvasiver Beatmung

Der erwartete Effekt der Abnahme der MSNA-Frequenz und Zunahme der Baroreflexsensitivität im Sinne einer Reduktion der Sympathoexzitation durch die nicht-invasive Beatmung war aus den vorliegenden Daten nicht nachweisbar. Weder die MSNA noch die Baroreflexsensitivität wurden durch die Anwendung von nichtinvasiver Beatmung signifikant beeinflusst. 


\section{Diskussion}

Im Rahmen der hier durchgeführten Studie konnte entsprechend der Hypothese 1 erstmals ein inverser Zusammenhang zwischen der maximalen körperlichen Leistungsfähigkeit von COPD-Patienten und dem Grad der sympathischen Aktivierung in Ruhe nachgewiesen werden.

Unter moderater körperlicher Belastung im Sinne statischer Handgrips ließ sich, wie in Hypothese 2 angenommen, bei den COPD-Patienten im Gegensatz zur Kontrollgruppe nochmals eine signifikante Steigerung der sympathischen Nervenaktivität messen.

Wie in Hypothese 3 vermutet, war zusätzlich der Anstieg der MSNA der COPDPatienten unter dynamischer Handgrip-Belastung negativ mit der maximalen körperlichen Leistungsfähigkeit korreliert. Dieser Effekt ließ sich in der Kontrollgruppe nicht reproduzieren.

Entgegen der anfänglichen Erwartungen ließ sich die sympatho-vagale Balance der COPD-Patienten weder in Ruhe noch unter moderater körperlicher Belastung durch den Einsatz nicht-invasiver Beatmung signifikant beeinflussen, sodass Hypothese 4 nicht bestätigt werden konnte.

\subsection{Teilnehmer-Charakteristika}

In der Lungenfunktion zeigten die COPD-Patienten wie erwartet signifikant schlechtere Werte als die gesunden Probanden. Insbesondere die Vitalkapazität und die $\mathrm{FEV}_{1}$ waren im Sinne einer Überblähung der Lunge und Obstruktion der Atemwege verändert. In der Kontrollgruppe lagen altersentsprechend normale Befunde vor.

Bezüglich der Herzfrequenz in Ruhe wichen die COPD-Patienten signifikant von der Kontrollgruppe ab. Bereits in früheren Studien wurde diskutiert, ob sich die gesteigerte Herzfrequenz bei COPD-Patienten auf die kardiale sympathische Aktivierung und die relative Suppression der vagalen Aktivität zurückführen lässt (Andreas et al. 2005) (Raupach et al. 2008). In der aktuellen Arbeit war - wie auch in den vorangegangenen - die Einnahme von Wirkstoffen mit direktem Effekt auf den Sympathikotonus ein Ausschlusskriterium. Somit ist ein Zusammenhang zwischen 
der beobachteten gesteigerten Herzfrequenz der COPD-Patienten und der hier ebenfalls gefundenen Sympathikus-Aktivierung weiterhin wahrscheinlich. Die Ursachen chronisch gesteigerter Herzfrequenz sollten bei der COPD auch im Hinblick auf die Entwicklung kardiovaskulärer Komorbiditäten weiter untersucht werden (Johansen et al. 2013).

Im Vergleich zur Kontrollgruppe zeigten die COPD-Patienten eine signifikant gesteigerte arterielle Pulswellengeschwindigkeit. Eine Steigerung der aPWV geht mit einer Nachlasterhöhung im Herz-Kreislaufsystem und einer verminderten Koronarperfusion einher und gilt als Risikofaktor für die Entstehung von HerzKreislauf-Erkrankungen (Maclay et al. 2009). Eine gesteigerte aPWV kann außerdem bei Rauchern gemessen werden, ohne dass eine COPD vorliegt. In der aktuellen Studie wurden COPD-Patienten und Kontrollprobanden nicht bezüglich des Rauchverhaltens gematcht, was möglicherweise einen Einfluss auf die Ergebnisse hat. In der Kontrollgruppe rauchte keiner der Probanden, während von den COPDPatienten zum Zeitpunkt der Untersuchung 6 von 30 Patienten rauchten. Weiter berichteten 21 COPD-Patienten und 6 gesunde Probanden, in der Vergangenheit geraucht zu haben. Unabhängig davon weisen die in dieser Studie erhobenen aPWV-Daten darauf hin, dass die Versteifung arterieller Gefäße ein pathophysiologisches Korrelat des epidemiologisch gesicherten Zusammenhangs zwischen dem Vorliegen einer COPD und dem Auftreten von Herz-KreislaufErkrankungen darstellen könnte.

Die Ruhe-MSNA der COPD-Patienten war signifikant höher als die der Kontrollgruppe. Dieser Effekt wurde bereits in vorausgegangenen Studien der Arbeitsgruppe sowohl für hypoxische Patienten (Heindl et al. 2001) als auch für normoxische Patienten (Raupach et al. 2008) dokumentiert und spiegelt die sympathische Aktivierung bei COPD wider. Erstmals konnte in der vorliegenden Arbeit gezeigt werden, dass das Ausmaß der sympatho-vagalen Imbalance in Ruhe bei COPD-Patienten mit NIV nochmals signifikant größer ist als bei COPD-Patienten ohne NIV.

Da die COPD-Patienten mit NIV in der Lungenfunktion deutlich schlechtere Werte erreichten als die COPD-Patienten ohne NIV, könnte dies ein Anhalt dafür sein, dass das Ausmaß der Sympathoexzitation in Ruhe mit dem Schweregrad der COPD 
korreliert. Um diesen Aspekt näher zu beleuchten, sind aber weiterführende Studien mit COPD-Patienten in allen Stadien der Erkrankung notwendig. Die Frequenz der Ruhe-MSNA in der aktuellen Studie (36,9 $\pm 2,1$ Bursts/min) lag zwar deutlich unter der Frequenz vorausgegangener Studien der Arbeitsgruppe (47,8 $\pm 4,9$ Bursts/min) (Raupach et al. 2008), die Differenz zwischen Kontrollgruppe und COPD erreichte aber ebenfalls statistische Signifikanz. Die Unterschiede in der MSNA-Frequenz zwischen den Studien lassen sich möglicherweise auf das vollständig neue Arrangement des Versuchsaufbaus zurückführen. Außerdem fiel auf, dass die adäquate MSNA-Ableitung umso schwieriger war, je ausgeprägter die COPD war.

Auch die Baroreflexsensitivität der COPD-Patienten war im Vergleich mit der Kontrollgruppe signifikant vermindert. Dies entspricht den Ergebnissen vorangegangener Studien mittels Spektralanalyse (Raupach et al. 2008) und Phenylephrinmethode (Patakas et al. 1982).

Insgesamt muss bei gesteigerter Herzfrequenz, gesteigerter MSNA und herabgesetzter Baroreflexsensitivität von einer bei COPD-Patienten bereits in Ruhe bestehenden Aktivierung des sympathischen Nervensystems ausgegangen werden. Die pathophysiologischen Mechanismen konnten bisher nicht eindeutig geklärt werden, sollten aber in Zukunft näher untersucht werden, um die Zusammenhänge pulmonaler und kardiovaskulärer Komorbiditäten besser zu verstehen.

Die Daten der Spiroergometrie lieferten signifikante Unterschiede zwischen COPDPatienten und gesunden Probanden. Die im Vergleich zur Kontrolle deutlich gesteigerte $\mathrm{VE} / \mathrm{VCO}_{2}$ kann als Ausdruck der respiratorischen Insuffizienz der COPDPatienten gewertet werden. Analog dazu lassen sich ähnliche Veränderungen auch in der Spiroergometrie von Herzinsuffizienz-Patienten nachweisen (Brunelli et al. 2010). Die Bestimmung der $\mathrm{VE} / \mathrm{VCO}_{2}$ liefert im Vergleich zu anderen Parametern $\left(\mathrm{VO}_{2} \max \right)$ deutlich validere Werte zur Bestimmung des Ventilations-PerfusionsMissmatches und eignet sich auch für schwerst-kompromittierte Patienten (Chua et al. 1997). 
Die maximale körperliche Leistung war bei den COPD-Patienten erheblich vermindert. Neben signifikanten Unterschieden zwischen COPD-Patienten und Kontrollgruppe ließen sich auch deutliche Differenzen zwischen COPD-Patienten mit und ohne Nutzung einer nicht-invasiven Beatmung dokumentieren. Bernard et al konnten nachweisen, dass das Ausmaß des Verlustes der Muskelmasse an der unteren Extremität bei COPD-Patienten mit dem Schweregrad der Erkrankung korreliert (Bernard et al. 1998). Dieser Zusammenhang von verminderter körperlicher Leistung und Schweregrad der COPD legt eine gegenseitige Beeinflussung von Inaktivität und dem Verlust von Muskelmasse im Sinne einer pulmonalen Kachexie nahe (Kim et al. 2008). Die Dauer der Belastung in der Spiroergometrie variierte allerdings von Patient zu Patient, da das Stufenprotokoll jeweils auf die individuelle Leistungsfähigkeit angepasst werden musste. Insgesamt stellt die Minderung der körperlichen Leistungsfähigkeit einen zentralen Aspekt der erhöhten Mortalität bei COPD-Patienten dar (Swallow et al. 2007), sodass hier ein möglicher Ansatzpunkt für neue Behandlungskonzepte liegen könnte.

Auch die maximale $\mathrm{O}_{2}$-Aufnahme korreliert mit dem Grad der COPD. Die von den COPD-Patienten ohne NIV (13,5 $\pm 1,3 \mathrm{l} / \mathrm{min})$ und mit NIV $(8,9 \pm 0,6 \mathrm{l} / \mathrm{min})$ erreichten Werte stellen hochgradige Einschränkungen der Ausdauerleistungsfähigkeit dar. Der Vergleich mit ähnlich eingeschränkten Herzinsuffizienz-Patienten unterstreicht das Ausmaß der körperlichen Beeinträchtigung durch die COPD zusätzlich. Bei Herzinsuffizienz-Patienten wird ab einer $\mathrm{VO}_{2} \max <14 \quad \mathrm{I} / \mathrm{min}$ und gleichzeitiger Einschränkung im täglichen Leben eine Herztransplantation in Erwägung gezogen, ab einer $\mathrm{VO}_{2} \max <10 \mathrm{l} / \mathrm{min}$ sogar dringend empfohlen (Mehra et al. 2006). Eine entsprechende adäquate Therapieoption zur Behandlung der COPD liegt zum jetzigen Zeitpunkt nicht vor.

Die weitere Diskussion der Ergebnisse erfolgt entsprechend der bereits in der Einleitung aufgestellten Hypothesen.

\subsection{Hypothese 1}

Die MSNA in Ruhe korreliert bei Gesunden und COPD-Patienten mit dem ventilatorischen Äquivalent für $\mathrm{CO}_{2}\left(\mathrm{VE} / \mathrm{VCO}_{2}\right)$, der maximalen $\mathrm{O}_{2}$-Aufnahme und der körperlichen Belastbarkeit in der Spiroergometrie auf dem Standfahrrad. 
Im gesamten Studienkollektiv bestand eine signifikante negative Korrelation zwischen der Ruhe-MSNA und der maximalen körperlichen Leistungsfähigkeit in der Spiroergometrie. In der aktuellen Studie konnte erstmals ein Zusammenhang von Sympathoexzitation und eingeschränkter körperlicher Leistung bei COPD nachgewiesen werden.

Die reduzierte körperliche Leistungsfähigkeit ist bei COPD-Patienten nicht nur mit einer gesteigerten Ruhe-MSNA, sondern auch mit einer erhöhten Mortalität verbunden. In aktuellen retrospektiven Studien (Rutten et al. 2010) (Short et al. 2011) konnte eine Senkung der Mortalität von COPD-Patienten durch den Einsatz von BBlockern beobachtet werden. Die Autoren führen diesen Effekt zum einen auf das gehäuft komorbide Auftreten der COPD und kardialer Erkrankungen zurück, allerdings scheint der beobachtete Effekt so groß zu sein, dass die Reduktion kardiovaskulärer Risikofaktoren alleine nicht als Erklärung ausreicht. Analog zur COPD lässt sich auch bei der Herzinsuffizienz eine neurohumorale Aktivierung nachweisen. Hier ist der Einsatz von Wirkstoffen, die neurohumorale Regelkreise modulieren, bereits seit Langem etablierter Bestandteil der Therapie. Der positive Effekt des Einsatzes von B-Blockern auf die Morbidität und Mortalität der COPD wurde auch beim Einsatz weiterer Wirkstoffe beobachtet, die ebenfalls einen Einfluss auf sympatho-vagale Regelkreise ausüben. So konnte in der Vergangenheit bereits eine signifikante Reduktion der Morbidität und Mortalität bei der COPD durch den Einsatz von Wirkstoffen, die das Renin-Angiotensin-II-System beeinflussen, nachgewiesen werden (Mortensen et al. 2009).

Insgesamt scheint sich also durch den Einsatz von B-Blockern und ACE-Hemmern bei COPD-Patienten - zusätzlich zur Reduktion kardio-vaskulärer Riskofaktoren die Modulation neuro-humoraler Regelkreise günstig auf den Verlauf der Erkrankung auszuwirken.

Einschränkend muss erwähnt werden, dass sich der Zusammenhang von reduzierter körperlicher Belastbarkeit und gesteigerter Ruhe-MSNA nicht für die einzelnen Teilgruppen nachweisen ließ, vermutlich aufgrund zu geringer Trennschärfe (Power). Der Endpunkt der Power-Analyse war die Identifikation von Unterschieden in der MSNA vor und nach Interventionen. Um statistisch signifikante Unterschiede 
zwischen den einzelnen Teilgruppen mit einer adäquaten Power identifizieren zu können, hätte das Studienkollektiv größer gewählt werden müssen.

Für die $\mathrm{VE} / \mathrm{VCO}_{2}$ erreichten die Korrelationen mit der Ruhe-MSNA zwar keine statistische Signifikanz, jedoch fällt auf, dass bei COPD-Patienten eine positive, bei der Kontrollgruppe hingegen eine negative Korrelation besteht.

In retrospektiven Studien konnte für Patienten mit chronischer Herzinsuffizienz ebenfalls eine gesteigerte $\mathrm{VE} / \mathrm{VCO}_{2}$ bei körperlicher Anstrengung und eine insgesamt verminderte Leistungsfähigkeit nachgewiesen werden (Chua et al. 1997).

Insgesamt tragen die Ergebnisse der vorliegenden Arbeit also zum Verständnis pathophysiologischer Zusammenhänge hinsichtlich der reduzierten Leistungsfähigkeit und der bereits in Ruhe bestehenden sympatho-vagalen Imbalance bei COPD-Patienten bei. Gleichzeitig passen die hier erhobenen Daten gut zu dem in der Literatur beschriebenen mortalitätssenkenden Effekt des Einsatzes von BBlockern bei COPD.

\subsection{Hypothese 2}

Die Steigerung der MSNA und die Abnahme der Baroreflexsensitivität während einer Handkontraktionsübung als Äquivalent leichter körperlicher Belastung sind bei COPD-Patienten größer als bei gesunden Probanden.

Während der dynamischen Handgrip-Belastung war die MSNA der COPD-Patienten signifikant höher als in der Kontrollgruppe. Es lag also nicht nur in Ruhe, sondern auch unter dynamischer Belastung ein signifikant höherer Sympathikotonus vor. Ähnliches konnte in der Vergangenheit bereits für Patienten mit chronischer Herzinsuffizienz nachgewiesen werden (Notarius et al. 2001).

Eine überschießende Reaktion mit signifikantem Anstieg der MSNA gegenüber den Ruhewerten ließ sich für diese Art der Belastung aber nicht nachweisen, das Ausmaß der sympatho-vagalen Imbalance scheint sich hier also nicht signifikant zu ändern. 
Im Gegensatz zu den dynamischen Handgrips reagierten die COPD-Patienten auf die statischen Handgrips mit einer signifikanten Steigerung der MSNA gegenüber den Werten in Ruhe. Auch dieser Effekt konnte in früheren Arbeiten bei Herzinsuffizienz-Patienten beobachtet werden (Notarius et al. 2001). Anders als in früheren Studien konnte in der vorliegenden Arbeit unter statischer HandgripBelastung keine signifikante Veränderung der MSNA in der Kontrollgruppe nachgewiesen werden (Mark et al. 1985).

Insgesamt scheint bei COPD-Patienten besonders die adäquate sympatho-vagale Reaktion auf statische Belastungen gestört zu sein. In welchem Ausmaß die bei COPD-Patienten beobachtete Umdifferenzierung der Skelettmuskulatur von Typ-IMuskelfasern zu schneller ermüdenden Typ-II-Muskelfasern eine Rolle spielt, ist zum jetzigen Zeitpunkt noch unklar (Kim et al. 2008).

Auch die Baroreflexsensitivität der COPD-Patienten war sowohl in Ruhe als auch unter statischer Belastung signifikant niedriger als in der Kontrollgruppe. Dies kann ebenfalls als Ausdruck des gestörten sympatho-vagalen Gleichgewichts gewertet werden. Bei Patienten mit chronischer Herzinsuffizienz ließ sich eine ähnlich veränderte Baroreflexsensitivität nachweisen. Hier konnte die Baroreflexsensitivität durch regelmäßiges körperliches Training signifikant gesteigert werden (lellamo et al. 2011).

Die Art der körperlichen Belastung scheint also einen wesentlichen Einfluss auf die sympatho-vagale Reaktion zu haben. Die Frage, ob der Ergoreflex der Muskulatur bei der COPD, ähnlich wie bei der chronischen Herzinsuffizienz, einen wesentlichen Einfluss auf die MSNA hat, kann auf der Grundlage der aktuell erhobenen Daten nicht abschließend geklärt werden, da auf die Durchführung einer Unterarmischämie verzichtet wurde. Diese sehr schmerzhafte Prozedur erschien bei den hier untersuchten schwerkranken Patienten nicht angezeigt. Die Überlegung, ob analog zur Herzinsuffizienz auch bei der COPD die Baroreflexsensitivität durch gezieltes Training verbessert und die Sympathoexzitation vermindert werden kann, bietet Ansatzpunkte für künftige Studien. 


\subsection{Hypothese 3}

Die Steigerung der MSNA und die Abnahme der Baroreflexsensitivität während der Handkontraktionsübungen korrelieren mit den in der Spiroergometrie erhobenen Daten.

Bei den COPD-Patienten ließ sich aus den vorliegenden Daten eine signifikante negative Korrelation der maximalen Leistungsfähigkeit mit dem Anstieg der MSNA unter dynamischer Belastung ableiten, während sich für die Kontrollgruppe kein signifikanter Zusammenhang ergab. Die bereits bei leichter körperlicher Belastung gemessene Sympathoexzitation scheint ein Faktor zu sein, der Ausdruck der Imbalance des vegetativen Nervensystems bei COPD-Patienten ist und das Ausmaß der Einschränkung der maximalen Leistungsfähigkeit beeinflusst. Der inverse Zusammenhang zwischen der Leistungsfähigkeit und der belastungsinduzierten Steigerung der MSNA ließ sich bei den COPD-Patienten nur für dynamische, nicht aber für statische Handgrips nachweisen. Eine mögliche Erklärung für diese Beobachtung könnte sein, dass die Belastung in der Spiroergometrie ebenfalls eher einer dynamischen Belastung entspricht, sodass hier ähnliche Belastungsformen vorlagen. Außerdem ist nicht auszuschließen, dass es unter den statischen Handgrips zu einer reflektorischen Anspannung der Bauchmuskulatur kam. Möglicherweise könnte die dabei entstehende Erhöhung des intraabdominellen Druckes eine kurzzeitige Blutdrucksteigerung mit entsprechender vagaler Aktivierung und sympathischer Inhibierung bedingt haben.

Die Korrelation zwischen der maximalen $\mathrm{O}_{2}$-Aufnahme und dem MSNA-Anstieg unter Belastung war in der aktuellen Studie nicht signifikant. Allerdings fällt auf, dass bei den COPD-Patienten eine negative, in der Kontrollgruppe hingegen eine positive Korrelation vorlag. Die $\mathrm{VO}_{2}$ max gibt zuverlässig die maximale aerobe Kapazität, also die Leistungsfähigkeit des Systems Lunge-Kreislauf-Muskelstoffwechsel an (Haber 2013). Die positive Korrelation in der Kontrollgruppe spiegelt physiologische Mechanismen einer adäquaten sympathischen Aktivierung bei im Vergleich zu den COPD-Patienten maximaler Leistung und großer körperlicher Leistungsfähigkeit wider. Die bei COPD-Patienten vorliegende Sympathoexzitation war mit einer limitierten maximalen aeroben Kapazität assoziiert. Analog zur chronischen Herzinsuffizienz scheint auch bei der COPD in der Anfangsphase der Erkrankung 
über den gesteigerten Sympathikotonus eine Kompensation der eingeschränkten körperlichen Belastbarkeit durch eine Aktivierung des Herz-Kreislauf-Systems stattzufinden. Im Verlauf der Erkrankung überwiegen aber schädigende Einflüsse wie die Blutdrucksteigerung im Lungenkreislauf und die daraus resultierende mögliche Entwicklung einer pulmonalen Hypertonie (Heindl et al. 2001). Der bei den COPDPatienten aktuell vorliegende inverse Zusammenhang von maximaler Leistungsfähigkeit des Systems Herz-Kreislauf-Muskel und dem MSNA-Anstieg unter moderater körperlicher Belastung kann als Ausdruck der inadäquaten neurohumoralen Kompensationsmechanismen bei fortgeschrittener COPD gewertet werden.

Auch die Korrelation zwischen der Änderung der Baroreflexsensitivität und der maximalen Leistung beziehungsweise der maximalen $\mathrm{O}_{2}$-Aufnahme erreichte keine statistische Signifikanz. Ähnlich wie für den MSNA-Anstieg unter Belastung lag bei den COPD-Patienten eine negative und für die Kontrollgruppe eine positive Korrelation vor.

Die bei COPD-Patienten beobachteten negativen Korrelationen zwischen der Änderung der Baroreflexsensitivität bzw. dem MSNA-Anstieg und den spiroergometrischen Parametern unterstreichen zusätzlich die Annahme, dass das Überwiegen des Sympathikotonus maßgeblich an der verminderten Leistungsfähigkeit beteiligt ist.

\subsection{Hypothese 4}

COPD-Patienten weisen nach der Benutzung nicht-invasiver Beatmung eine niedrigere MSNA und eine höhere Baroreflexsensitivität in Ruhe und unter leichter körperlicher Belastung auf als vor der Benutzung.

Für den Vergleich der MSNA der COPD-Patienten mit NIV unter leichter Belastung vor und nach der Benutzung der nicht-invasiven Beatmung ließ sich in den vorliegenden Daten keine signifikante Differenz dokumentieren. Diese Beobachtung deckt sich mit den Ergebnissen anderer Studien, die eine Sympathikusaktivierung 
aufgrund negativer hämodynamischer Effekte durch die Änderung der intrathorakalen Druckverhältnisse während des Einsatzes von cPAP-Beatmung diskutieren (Lukacsovits et al. 2012). Bereits in früheren Publikationen der Arbeitsgruppe wurde eine gesteigerte MSNA durch den Einsatz von CPAP bei Patienten mit chronischer Herzinsuffizienz festgestellt (Heindl et al. 2001). Der Effekt scheint auf einer Hemmung aortaler Barorezeptoren zu beruhen. Der Einsatz positiver Atemwegsdrücke bedingt eine intrapleurale und intrathorakale Drucksteigerung. Die Druckdifferenz über der Wand der Aorta ist durch die Steigerung des intrathorakalen Druckes im Rahmen der Beatmung insgesamt vermindert, da der intrathorakale Druck in Richtung des intravasalen Druckes verschoben wird. Die intraaortalen Druckverhältnisse entsprechen daher einer hypotonen Kreislauflage.

Gleichzeitig ist durch die intrathorakale Drucksteigerung die Füllung des rechten Vorhofes des Herzens vermindert. Atriale Barorezeptoren werden gehemmt und bedingen eine weitere Steigerung des Sympathikotonus. Je höher die Beatmungsdrücke sind, desto ausgeprägter ist die negative hämodynamische Wirkung der Beatmung (Lukacsovits et al. 2012). Diesen Effekten kann nach derzeitigem Verständnis nur adäquat durch eine Steigerung des Sympathikotonus entgegengewirkt werden.

Die enge funktionale und anatomische Verknüpfung von Lunge und HerzKreislaufsystem erschwert zusätzlich eine isolierte Betrachtung der Teilsysteme.

Eine mögliche Reduktion des Sympathikotonus durch die Entlastung der Atemmuskulatur könnte also durch sympathoexzitatorische Wirkungen auf das HerzKreislaufsystem maskiert werden. Außerdem muss in Betracht gezogen werden, dass der 20-minütige Einsatz der Beatmung möglicherweise nicht ausreichend lang ist, um eine relevante Entlastung der Atemmuskulatur zu erzielen. Idealerweise hätte die Messung morgens direkt nach nächtlicher Nutzung der Beatmung durchgeführt werden sollen. Allerdings passen die hier dargestellten Ergebnisse zu der Erkenntnis, dass eine kurzzeitige Belastung der Atemmuskulatur durch inspiratorische Widerstände die sympathische Aktivität nicht steigert (Raupach et al. 2010). 


\subsection{Stärken- und Schwächenanalyse}

Obwohl in der vorliegenden Arbeit mehrere zum Teil zentrale pathophysiologische Mechanismen der COPD identifiziert wurden, erreichte ein Teil der Ergebnisse, ungeachtet ihrer möglichen klinischen Relevanz, keine statistische Signifikanz.

Die Mikroneurographie stellt eine technisch anspruchsvolle Methode zur Darstellung der Aktivität des vegetativen Nervensystems dar. Auch aufgrund der vielfachen Verstärkung des Signals liegen bei dieser Methode viele Störvariablen vor, die sowohl die Aufzeichnung als auch die Auswertung der Messwerte erschwert. Da der Versuchsaufbau für die vorliegende Studie vollständig neu zusammengestellt werden musste und auch die Untersucher neu eingearbeitet wurden, unterlag die Qualität des Nervensignals insbesondere zu Beginn der Studie starken Schwankungen.

Auch der Einsatz neuer Hard- und Software erschwerte anfangs die Auswertung der MSNA-Signale, da der Versuchsaufbau erst im Verlauf optimiert werden konnte. Sowohl die Messwerte der Kontrollgruppe als auch der COPD-Patienten lieferten zwar die gleiche Tendenz wie in vorausgegangenen Studien der Arbeitsgruppe, die absoluten Werte wichen aber teilweise von den Vorergebnissen ab (Raupach et al. 2008).

Letztlich ist nicht auszuschließen, dass auch die Länge der Untersuchung bei schwerst-kompromittierten Patienten zu einer geringfügigen Sympathoexzitation im Verlauf beigetragen hat.

Gleichzeitig wurde in der vorliegenden Arbeit aber eine Vielzahl physiologisch relevanter Parameter der körperlichen Leistung und des autonomen Nervensystems erhoben, sodass sich ein umfassendes Bild der MSNA bei COPD-Patienten in Ruhe und unter Belastung sowie unter nicht-invasiver Beatmung ergibt.

Weiter wurde während der Rekrutierung sichergestellt, dass gesunde Probanden und COPD-Patienten sich hinsichtlich entscheidender Basisvariablen nicht signifikant unterschieden. Aufgrund der hohen Übereinstimmung der Gruppen (siehe auch Tabelle 4) waren ein detaillierter Vergleich der Messwerte und eine Minimierung von Störvariablen möglich.

Insgesamt wurden in der vorliegenden Arbeit erstmals Zusammenhänge der COPD, der Sympathoexzitation und der reduzierten körperlichen Leistungsfähigkeit untersucht. Die erhobenen Daten eröffnen neue Einblicke und ausreichend Anlass zu weiterführenden Studien. 


\section{$5 \quad$ Zusammenfassung}

Die COPD ist die weltweit vierthäufigste Todesursache und hat weitreichende sozioökonomische Konsequenzen. Ein wesentlicher pathophysiologischer Aspekt der Erkrankung ist eine Imbalance des autonomen Nervensystems, die neben inflammatorischen Prozessen das Auftreten extrapulmonaler Manifestationen der COPD begünstigt. Insofern besteht eine Parallele zur systolischen Herzinsuffizienz, bei der ebenfalls eine Überaktivität des sympathischen Nervensystems festgestellt wurde. Entsprechend greifen viele der in den aktuellen Leitlinien für die Behandlung der Herzinsuffizienz empfohlenen Medikamente (z.B. Betablocker) in die sympathovagale Balance ein. Analog zur Herzinsuffizienz besteht auch bei der COPD ein Zusammenhang zwischen der Einschränkung der körperlichen Leistungsfähigkeit und der Mortalität. Die zugrundeliegenden pathophysiologischen Mechanismen sind jedoch noch nicht ausreichend erforscht.

An 30 Patienten mit chronisch obstruktiver Lungenerkrankung, davon 15 Patienten mit nicht-invasiver Beatmung (NIV) und 15 gesunden Probanden, wurden als Marker für die sympatho-vagale Balance die muskelsympathische Nervenaktivität (MSNA) und die Baroreflexsensitivität bestimmt. Während der jeweils einstündigen Untersuchungen wurde der Einfluss einer moderaten körperlichen Belastung sowie der nicht-invasiven Beatmung auf diese Parameter untersucht. Zudem wurden während der Versuche kontinuierlich Herzfrequenz, Blutdruck, Thoraxexkursionen und die transkutane Sauerstoff- und Kohlenstoffdioxidkonzentration gemessen. Zur Beurteilung der Leistungsfähigkeit unterzogen sich alle Studienteilnehmer vor den Messungen einer Spiroergometrie.

In Ruhe ergab sich für die COPD-Patienten eine im Vergleich zu den Kontrollprobanden signifikant gesteigerte MSNA und eine signifikant reduzierte Baroreflexsensitivität. Dabei bestand in der Gesamtgruppe aller untersuchten Personen ein inverser Zusammenhang zwischen der maximalen ergometrischen Leistungsfähigkeit und dem Ausmaß der Sympathoexzitation in Ruhe. 
Eine körperliche Belastung mit statischen Handgrips bedingte eine signifikante Steigerung der MSNA bei den COPD-Patienten, nicht aber bei den gesunden Kontrollprobanden. Dieser Anstieg der sympathischen Aktivität wies eine negative (allerdings nicht signifikante) Korrelation mit der maximalen Leistungsfähigkeit auf. Eine dynamische Belastung blieb in beiden Gruppen ohne Auswirkungen auf die MSNA. Weder bei Gesunden noch bei Patienten führten die beiden Belastungsformen zu einer signifikanten Änderung der Baroreflexsensitivität. Der kurzzeitige Einsatz von NIV zeigte ebenfalls keinen signifikanten Effekt auf die MSNA oder die Baroreflexsensitivität.

Diese Ergebnisse bestätigen Beobachtungen zur MSNA bei COPD vorangegangener Studien. Darüber hinaus konnte erstmals ein Zusammenhang von sympatho-vagaler Imbalance und reduzierter körperlicher Leistungsfähigkeit bei COPD-Patienten dokumentiert werden. Zusätzlich konnte bei den COPD-Patienten im Gegensatz zur Kontrollgruppe eine Sympathoexzitation bereits auf moderate statische Belastung gezeigt werden. Weiter ergibt sich aus den gewonnenen Daten eine mögliche Erklärung für die Mortalitätssenkung bei COPD durch den Einsatz von B-Blockern. Die enge Verknüpfung von gesteigerter MSNA und Mortalität der COPD legt nahe, dass die medikamentöse Blockade des Sympathikus eine Mortalitätssenkung bedingt. Der tatsächliche Gewinn daraus sollte in einer randomisierten klinischen Studie genauer untersucht werden.

Der fehlende Nachweis einer Modulation sowohl der Ruhe-MSNA als auch der belastungsinduzierten MSNA-Zunahme unter nicht-invasiver Beatmung ist einerseits durch eine eventuelle Co-Aktivierung aortaler Barorezeptoren bei NIV erklärbar. Andererseits könnte dieses negative Ergebnis aber auch Folge der zu geringen Trennschärfe in der Subgruppen-Analyse gewesen sein. 


\section{$6 \quad$ Literaturverzeichnis}

(ATS) ATS (1995): Standards for the diagnosis and care of patients with chronic obstructive pulmonary disease. American Thoracic Society. Am J Respir Crit Care Med 152, S77-121

Agusti A, Soriano JB (2008): COPD as a systemic disease. COPD $\underline{5}, 133-138$

Andreas S, Reiter H, Luthje L, Delekat A, Grunewald RW, Hasenfuss G, Somers VK (2004): Differential effects of theophylline on sympathetic excitation, hemodynamics, and breathing in congestive heart failure. Circulation $\underline{110}$, 2157-2162

Andreas S, Anker SD, Scanlon PD, Somers VK (2005): Neurohumoral activation as a link to systemic manifestations of chronic lung disease. Chest $\underline{128}$, 3618-3624

Anthonisen NR, Connett JE, Kiley JP, Altose MD, Bailey WC, Buist AS, Conway WA, Jr., Enright PL, Kanner RE, O'Hara P (1994): Effects of smoking intervention and the use of an inhaled anticholinergic bronchodilator on the rate of decline of FEV1. The Lung Health Study. JAMA 272, 1497-1505

Anthonisen NR, Connett JE, Murray RP (2002): Smoking and lung function of Lung Health Study participants after 11 years. Am J Respir Crit Care Med 166, 675679

Anthonisen NR, Skeans MA, Wise RA, Manfreda J, Kanner RE, Connett JE (2005): The effects of a smoking cessation intervention on 14.5-year mortality: a randomized clinical trial. Ann Intern Med 142, 233-239

Barnes PJ, Celli BR (2009): Systemic manifestations and comorbidities of COPD. Eur Respir J 33, 1165-1185

Barreiro E, de la Puente B, Minguella J, Corominas JM, Serrano S, Hussain SN, Gea $J$ (2005): Oxidative stress and respiratory muscle dysfunction in severe chronic obstructive pulmonary disease. Am J Respir Crit Care Med 171, 11161124

Bernard S, LeBlanc P, Whittom F, Carrier G, Jobin J, Belleau R, Maltais F (1998): Peripheral muscle weakness in patients with chronic obstructive pulmonary disease. Am J Respir Crit Care Med 158, 629-634

Brunelli A, Pompili C, Belardinelli R (2010): Beyond peak VO2: ventilatory inefficiency (VE/VCO2 slope) measured during cardiopulmonary exercise test to refine risk stratification in lung resection candidates. Eur J Cardiothorac Surg 38, 19-20

Butler J, Schrijen F, Henriquez A, Polu JM, Albert RK (1988): Cause of the raised wedge pressure on exercise in chronic obstructive pulmonary disease. Am Rev Respir Dis 138, 350-354

Calverley PM, Burge PS, Spencer S, Anderson JA, Jones PW (2003): Bronchodilator reversibility testing in chronic obstructive pulmonary disease. Thorax $\underline{58}, 659$ 664

Calverley PM, Sanchez-Toril F, Mclvor A, Teichmann P, Bredenbroeker D, Fabbri LM (2007): Effect of 1-year treatment with roflumilast in severe chronic obstructive pulmonary disease. Am J Respir Crit Care Med 176, 154-161

Chappell S, Daly L, Morgan K, Guetta Baranes T, Roca J, Rabinovich R, Millar A, Donnelly SC, Keatings V, MacNee W (2006): Cryptic haplotypes of SERPINA1 
confer susceptibility to chronic obstructive pulmonary disease. Hum Mutat $\underline{27}$, 103-109

Charkoudian N, Rabbitts JA (2009): Sympathetic neural mechanisms in human cardiovascular health and disease. Mayo Clin Proc $\underline{84}$, 822-830

Chen JC, Mannino DM (1999): Worldwide epidemiology of chronic obstructive pulmonary disease. Curr Opin Pulm Med $\underline{5}, 93-99$

Chua TP, Ponikowski P, Harrington D, Anker SD, Webb-Peploe K, Clark AL, PooleWilson PA, Coats AJ (1997): Clinical correlates and prognostic significance of the ventilatory response to exercise in chronic heart failure. J Am Coll Cardiol $\underline{29}, 1585-1590$

Cooper CB (2006): The connection between chronic obstructive pulmonary disease symptoms and hyperinflation and its impact on exercise and function. Am J Med 119, 21-31

Crisafulli A, Scott AC, Wensel R, Davos CH, Francis DP, Pagliaro P, Coats AJ, Concu A, Piepoli MF (2003): Muscle metaboreflex-induced increases in stroke volume. Med Sci Sports Exerc 35, 221-228; discussion 229

de Torres JP, Pinto-Plata V, Casanova C, Mullerova H, Cordoba-Lanus E, Muros de Fuentes M, Aguirre-Jaime A, Celli BR (2008): C-reactive protein levels and survival in patients with moderate to very severe COPD. Chest $\underline{133}, 1336-$ 1343

Delius W, Hagbarth KE, Hongell A, Wallin BG (1972): General characteristics of sympathetic activity in human muscle nerves. Acta Physiol Scand 84, 65-81

Di Francia M, Barbier D, Mege JL, Orehek J (1994): Tumor necrosis factor-alpha levels and weight loss in chronic obstructive pulmonary disease. Am J Respir Crit Care Med 150, 1453-1455

Geldmacher H, Biller H, Herbst A, Urbanski K, Allison M, Buist AS, Hohlfeld JM, Welte $T$ (2008): [The prevalence of chronic obstructive pulmonary disease (COPD) in Germany. Results of the BOLD study]. Dtsch Med Wochenschr $133,2609-2614$

Group N (1980): Continuous or nocturnal oxygen therapy in hypoxemic chronic obstructive lung disease: a clinical trial. Nocturnal Oxygen Therapy Trial Group. Ann Intern Med 93, 391-398

Gulbas G, Gunen H, In E, Kilic T (2012): Long-term follow-up of chronic obstructive pulmonary disease patients on long-term oxygen treatment. Int $\mathrm{J}$ Clin Pract $\underline{66}, 152-157$

Haber $P$ (Hrsg.): in: Lungenfunktion und Spiroergometrie: Interpretation und Befunderstellung unter Einschluss der arteriellen Blutgasanalyse, Springer Verlag, Vienna 2013, $128 \mathrm{ff}$

Hagbarth KE, Vallbo AB (1968): Pulse and respiratory grouping of sympathetic impulses in human muscle-nerves. Acta Physiol Scand 74, 96-108

Halbert RJ, Natoli JL, Gano A, Badamgarav E, Buist AS, Mannino DM (2006): Global burden of COPD: systematic review and meta-analysis. Eur Respir J 28, 523532

Han MK (2011): Update in chronic obstructive pulmonary disease in 2010. Am J Respir Crit Care Med 183, 1311-1315

Hansen J, Sander M (2003): Sympathetic neural overactivity in healthy humans after prolonged exposure to hypobaric hypoxia. J Physiol $\underline{546}, 921-929$ 
He Y, Jiang B, Li LS, Ko L, Wu L, Sun DL, He SF, Liang BQ, Hu FB, Lam TH (2012): Secondhand smoke exposure predicted COPD and other tobacco-related mortality in a 17-year cohort study in China. Chest 142, 909-918

Heindl S, Dodt C, Krahwinkel M, Hasenfuss G, Andreas S (2001): Short term effect of continuous positive airway pressure on muscle sympathetic nerve activity in patients with chronic heart failure. Heart $\underline{85}, 185-190$

Heindl S, Lehnert M, Criee CP, Hasenfuss G, Andreas S (2001): Marked sympathetic activation in patients with chronic respiratory failure. Am $\mathrm{J}$ Respir Crit Care Med 164, 597-601

lellamo F, Manzi V, Caminiti G, Sposato B, Massaro M, Cerrito A, Rosano G, Volterrani M (2011): Dose-response relationship of baroreflex sensitivity and heart rate variability to individually-tailored exercise traininh in patients with heart failure. Int J Cardiol 166, 334-339

Jänig W: Vegetatives Nervensystem; in: Physiologie des Menschen, 30. überarbeitete Auflage; hrsg. v. Schmidt RF, Lang F; Springer, Berlin 2007, 440-452

Janssen SP, Gayan-Ramirez G, Van den Bergh A, Herijgers P, Maes K, Verbeken E, Decramer M (2005): Interleukin-6 causes myocardial failure and skeletal muscle atrophy in rats. Circulation $\underline{111}, 996-1005$

Johansen CD, Olsen RH, Pedersen LR, Kumarathurai P, Mouridsen MR, Binici Z, Intzilakis T, Kober L, Sajadieh A (2013): Resting, night-time, and 24 h heart rate as markers of cardiovascular risk in middle-aged and elderly men and women with no apparent heart disease. Eur Heart J 34, 1732-1739

Juarez SP, Merlo J (2013): Revisiting the effect of maternal smoking during pregnancy on offspring birthweight: a quasi-experimental sibling analysis in Sweden. PLoS One $\underline{8}$, e61734

Kerstjens HA, Rijcken B, Schouten JP, Postma DS (1997): Decline of FEV1 by age and smoking status: facts, figures, and fallacies. Thorax $\underline{52}, 820-827$

Kim HC, Mofarrahi M, Hussain SN (2008): Skeletal muscle dysfunction in patients with chronic obstructive pulmonary disease. Int $\mathrm{J}$ Chron Obstruct Pulmon Dis 3, 637-658

Konietzko N, Fabel H (Hrsg.): in: Weissbuch Lunge 2000 : die Lunge und ihre Erkrankungen : zur Lage und Zukunft der Pneumologie in Deutschland, Georg Thieme, Stuttgart 2000,

Lambert EA, Schlaich MP, Dawood T, Sari C, Chopra R, Barton DA, Kaye DM, Elam M, Esler MD, Lambert GW (2011): Single-unit muscle sympathetic nervous activity and its relation to cardiac noradrenaline spillover. J Physiol 589, 25972605

Lee H, Kim J, Tagmazyan K (2013): Treatment of Stable Chronic Obstructive Pulmonary Disease: the GOLD Guidelines. Am Fam Physician 88, 655-663

Levine S, Kaiser L, Leferovich J, Tikunov B (1997): Cellular adaptations in the diaphragm in chronic obstructive pulmonary disease. N Engl J Med 337, 17991806

Loewy AD, Spyer KM (Hrsg.): in: Central regulation of autonomic functions, Oxford University Press, New York 1990,

Lukacsovits J, Carlucci A, Hill N, Ceriana P, Pisani L, Schreiber A, Pierucci P, Losonczy G, Nava S (2012): Physiological changes during low- and highintensity noninvasive ventilation. Eur Respir J $\underline{39}$, 869-875 
Maclay JD, McAllister DA, Mills NL, Paterson FP, Ludlam CA, Drost EM, Newby DE, Macnee W (2009): Vascular dysfunction in chronic obstructive pulmonary disease. Am J Respir Crit Care Med 180, 513-520

Mark AL, Victor RG, Nerhed C, Wallin BG (1985): Microneurographic studies of the mechanisms of sympathetic nerve responses to static exercise in humans. Circ Res $\underline{57}, 461-469$

Meecham Jones DJ, Paul EA, Jones PW, Wedzicha JA (1995): Nasal pressure support ventilation plus oxygen compared with oxygen therapy alone in hypercapnic COPD. Am J Respir Crit Care Med 152, 538-544

Mehra MR, Kobashigawa J, Starling R, Russell S, Uber PA, Parameshwar J, Mohacsi P, Augustine S, Aaronson K, Barr M (2006): Listing criteria for heart transplantation: International Society for Heart and Lung Transplantation guidelines for the care of cardiac transplant candidates--2006. J Heart Lung Transplant 25, 1024-1042

Mills NL, Miller JJ, Anand A, Robinson SD, Frazer GA, Anderson D, Breen L, Wilkinson IB, McEniery CM, Donaldson K (2008): Increased arterial stiffness in patients with chronic obstructive pulmonary disease: a mechanism for increased cardiovascular risk. Thorax $\underline{63}, 306-311$

Montes de Oca M, Celli BR (2000): Respiratory muscle recruitment and exercise performance in eucapnic and hypercapnic severe chronic obstructive pulmonary disease. Am J Respir Crit Care Med 161, 880-885

Morgan BJ, Crabtree DC, Palta M, Skatrud JB (1995): Combined hypoxia and hypercapnia evokes long-lasting sympathetic activation in humans. J Appl Physiol $\underline{79}$, 205-213

Morgan MD (1991): Experience of using the CRQ (Chronic Respiratory Questionnaire). Respir Med 85 Suppl B, 23-24; discussion 33-27

Mortensen EM, Copeland LA, Pugh MJ, Restrepo MI, de Molina RM, Nakashima B, Anzueto A (2009): Impact of statins and ACE inhibitors on mortality after COPD exacerbations. Respir Res 10, 45

Murray CJ, Lopez AD (1997): Alternative projections of mortality and disability by cause 1990-2020: Global Burden of Disease Study. Lancet 349, 1498-1504

Notarius CF, Atchison DJ, Floras JS (2001): Impact of heart failure and exercise capacity on sympathetic response to handgrip exercise. Am J Physiol Heart Circ Physiol 280, H969-976

O'Donnell DE (2006): Hyperinflation, dyspnea, and exercise intolerance in chronic obstructive pulmonary disease. Proc Am Thorac Soc $\underline{3}, 180-184$

Osterziel KJ, Hanlein D, Willenbrock R, Eichhorn C, Luft F, Dietz R (1995): Baroreflex sensitivity and cardiovascular mortality in patients with mild to moderate heart failure. Br Heart J $\underline{73}, 517-522$

Patakas D, Louridas G, Kakavelas E (1982): Reduced baroreceptor sensitivity in patients with chronic obstructive pulmonary disease. Thorax 37, 292-295

Pauwels RA, Buist AS, Calverley PM, Jenkins CR, Hurd SS (2001): Global strategy for the diagnosis, management, and prevention of chronic obstructive pulmonary disease. NHLBI/WHO Global Initiative for Chronic Obstructive Lung Disease (GOLD) Workshop summary. Am J Respir Crit Care Med 163, 12561276 
Pellegrino R, Viegi G, Brusasco V, Crapo RO, Burgos F, Casaburi R, Coates A, van der Grinten CP, Gustafsson P, Hankinson J (2005): Interpretative strategies for lung function tests. Eur Respir J 26, 948-968

Pillai SG, Kong X, Edwards LD, Cho MH, Anderson WH, Coxson HO, Lomas DA, Silverman EK (2010): Loci identified by genome-wide association studies influence different disease-related phenotypes in chronic obstructive pulmonary disease. Am J Respir Crit Care Med 182, 1498-1505

Pirie K, Peto R, Reeves GK, Green J, Beral V (2012): The 21st century hazards of smoking and benefits of stopping: a prospective study of one million women in the UK. Lancet 381, 133-141

Prescott E, Bjerg AM, Andersen PK, Lange P, Vestbo J (1997): Gender difference in smoking effects on lung function and risk of hospitalization for COPD: results from a Danish longitudinal population study. Eur Respir J 트, 822-827

Prescott E, Lange P, Vestbo J (1999): Socioeconomic status, lung function and admission to hospital for COPD: results from the Copenhagen City Heart Study. Eur Respir J 13, 1109-1114

Raupach T, Bahr F, Herrmann P, Luethje L, Heusser K, Hasenfuss G, Bernardi L, Andreas $S$ (2008): Slow breathing reduces sympathoexcitation in COPD. Eur Respir J 32, 387-392

Raupach T, Bahr F, Herrmann P, Luthje L, Hasenfuss G, Andreas S (2010): Inspiratory resistive loading does not increase sympathetic tone in COPD. Respir Med 104, 107-113

Rennard SI, Fogarty C, Kelsen S, Long W, Ramsdell J, Allison J, Mahler D, Saadeh C, Siler T, Snell P (2007): The safety and efficacy of infliximab in moderate to severe chronic obstructive pulmonary disease. Am J Respir Crit Care Med $\underline{175}, 926-934$

Rutten FH, Zuithoff NP, Hak E, Grobbee DE, Hoes AW (2010): Beta-blockers may reduce mortality and risk of exacerbations in patients with chronic obstructive pulmonary disease. Arch Intern Med 170, 880-887

Scanlon PD, Connett JE, Waller LA, Altose MD, Bailey WC, Buist AS (2000): Smoking cessation and lung function in mild-to-moderate chronic obstructive pulmonary disease. The Lung Health Study. Am J Respir Crit Care Med 161, 381-390

Shaheen SO, Barker DJ, Shiell AW, Crocker FJ, Wield GA, Holgate ST (1994): The relationship between pneumonia in early childhood and impaired lung function in late adult life. Am J Respir Crit Care Med 149, 616-619

Short PM, Lipworth SI, Elder DH, Schembri S, Lipworth BJ (2011): Effect of beta blockers in treatment of chronic obstructive pulmonary disease: a retrospective cohort study. BMJ 342, d2549

Shrikrishna D, Patel M, Tanner RJ, Seymour JM, Connolly BA, Puthucheary ZA, Walsh SL, Bloch SA, Sidhu PS, Hart N (2012): Quadriceps wasting and physical inactivity in patients with COPD. Eur Respir $\mathrm{J}$

Siafakas NM, Vermeire P, Pride NB, Paoletti P, Gibson J, Howard P, Yernault JC, Decramer M, Higenbottam T, Postma DS (1995): Optimal assessment and management of chronic obstructive pulmonary disease (COPD). The European Respiratory Society Task Force. Eur Respir J $\underline{8}$, 1398-1420

Spruit MA, Gosselink R, Troosters T, Kasran A, Gayan-Ramirez G, Bogaerts P, Bouillon R, Decramer M (2003): Muscle force during an acute exacerbation in 
hospitalised patients with COPD and its relationship with CXCL8 and IGF-I. Thorax $\underline{58}$, 752-756

Spyer KM (1982): Central nervous integration of cardiovascular control. J Exp Biol $\underline{100}, 109-128$

Stewart AG, Waterhouse JC, Billings CG, Baylis P, Howard P (1994): Effects of angiotensin converting enzyme inhibition on sodium excretion in patients with hypoxaemic chronic obstructive pulmonary disease. Thorax $\underline{49}, 995-998$

Swallow EB, Reyes D, Hopkinson NS, Man WD, Porcher R, Cetti EJ, Moore AJ, Moxham J, Polkey MI (2007): Quadriceps strength predicts mortality in patients with moderate to severe chronic obstructive pulmonary disease. Thorax $\underline{62}, 115-120$

Tashkin DP, Rennard S, Hays JT, Ma W, Lawrence D, Lee TC (2011): Effects of varenicline on smoking cessation in patients with mild to moderate COPD: a randomized controlled trial. Chest $\underline{139}, 591-599$

Thurlbeck WM, Simon G (1978): Radiographic appearance of the chest in emphysema. AJR Am J Roentgenol 130, 429-440

Tracey KJ, Wei H, Manogue KR, Fong Y, Hesse DG, Nguyen HT, Kuo GC, Beutler B, Cotran RS, Cerami A (1988): Cachectin/tumor necrosis factor induces cachexia, anemia, and inflammation. J Exp Med 167, 1211-1227

Vrieze A, de Greef MH, Wijkstra PJ, Wempe JB (2007): Low bone mineral density in COPD patients related to worse lung function, low weight and decreased fatfree mass. Osteoporos Int $\underline{18}, 1197-1202$

Wallin G, Delius W, Lofstedt L, Sundlof G (1973): Baroreflex Control of Sympathetic Outflow to Skeletal-Muscle in Man. Acta Physiologica Scandinavica 93-93

Yamakoshi KI, Shimazu H, Togawa T (1980): Indirect measurement of instantaneous arterial blood pressure in the human finger by the vascular unloading technique. IEEE Trans Biomed Eng 27, 150-155

Yucha CB (2000): Use of microneurography to evaluate sympathetic activity in hypertension: a brief review. Appl Psychophysiol Biofeedback $\underline{25}, 55-63$ 


\section{$7 \quad$ Abbildungsverzeichnis}

Abbildung 1: Fluss-Volumen-Kurve bei COPD (rot). Die schwarze Kurve stellt die Idealform bei Gesunden dar. 5

Abbildung 2: 20-sekündiger Ausschnitt aus der kontinuierlichen simultanen Aufzeichnung des Elektrokardiogramms (grün) und des integrierten MSNA-Signals (rot) bei einem COPD-Patienten; Im unteren Teil vergrößertes 7-sekündiges Intervall 36

Abbildung 3: MSNA in Ruhe im Vergleich der drei Studiengruppen. Die Werte in den Säulen geben den Mittelwert mit Standardfehler an. Der angegebene $p$-Wert bezieht sich auf den zweiseitigen Student`s t-Test für gepaarte Stichproben. 37 Abbildung 4: Baroreflexsensitivität in Ruhe im Vergleich der drei Studiengruppen. Die Werte in den Säulen geben den Mittelwert mit Standardfehler an. Der angegebene pWert bezieht sich auf den zweiseitigen Student`s t-Test für gepaarte Stichproben. . 38 


\section{Tabellenverzeichnis}

Tabelle 1: Schweregradeinschätzung der COPD in der Spirometrie (Vestbo et al. 2013) 9

Tabelle 2: Stadieneinteilung der COPD (Vestbo et al. 2013) ................................... 9

Tabelle 3: Stufenschema der COPD-Therapie im Intervall (Vestbo et al. 2013)....... 11

Tabelle 4: Baseline-Charakteristika der 45 Studienteilnehmer ............................... 35

Tabelle 5: MSNA bei COPD und Kontrolle ………......................................... 37

Tabelle 6: Spiroergometrie bei COPD und Kontrolle ............................................. 39

Tabelle 7: Korrelation von MSNA und Spiroergometrie ....................................... 41

Tabelle 8: MSNA [Bursts/min] in Ruhe und unter dynamischer Belastung ............... 42

Tabelle 9: MSNA [Bursts/min] in Ruhe und unter statischer Belastung ................... 42

Tabelle 10: Baroreflexsensitivität $[\mathrm{ms} / \mathrm{mmHg}]$ in Ruhe und unter dynamischer Belastung.

Tabelle 11: Baroreflexsensitivität [ms/mmHg] in Ruhe und unter statischer Belastung 43

Tabelle 12: Korrelation von max. Leistungsfähigkeit mit Anstieg der MSNA …........ 44

Tabelle 13: Korrelation von max. $\mathrm{O}_{2}$-Aufnahme mit Anstieg der MSNA ................... 45

Tabelle 14:Korrelation von max. Leistungsfähigkeit mit Differenz der BRS .............. 46

Tabelle 15:Korrelation von max. $\mathrm{O}_{2}$-Aufnahme mit Differenz der BRS .................... 46

Tabelle 16: MSNA [Bursts/min] vor und nach Anwendung nicht-invasiver Beatmung

Tabelle 17: Baroreflexsensitivität $[\mathrm{ms} / \mathrm{mmHg}]$ vor und nach Anwendung nichtinvasiver Beatmung 48 